\title{
Data Compilation for AGR-3/4 Matrix Ring Blank Lot RDKRS
}

\author{
John D. Hunn, Michael P. Trammell, \\ and Fred C. Montgomery
}

\section{Revision 1}

September 2011

\author{
Prepared for the \\ United States Department of Energy - \\ Office of Nuclear Energy \\ under the \\ Next Generation Nuclear Plant - \\ Advanced Gas Reactor Fuel Development Program
}




\section{DOCUMENT AVAILABILITY}

Reports produced after January 1, 1996, are generally available free via the U.S. Department of Energy (DOE) Information Bridge.

Web site http://www.osti.gov/bridge

Reports produced before January 1, 1996, may be purchased by members of the public from the following source.

National Technical Information Service 5285 Port Royal Road

Springfield, VA 22161

Telephone 703-605-6000 (1-800-553-6847)

TDD 703-487-4639

Fax 703-605-6900

E-mailinfo@ntis.fedworld.gov

Web site http://www.ntis.gov/support/ordernowabout.htm

Reports are available to DOE employees, DOE contractors, Energy Technology Data Exchange (ETDE) representatives, and International Nuclear Information System (INIS) representatives from the following source.

Office of Scientific and Technical Information P.O. Box 62

Oak Ridge, TN 37831

Telephone 865-576-8401

Fax 865-576-5728

E-mail reports@adonis.osti.gov

Web site http://www.osti.gov/contact.html

This report was prepared as an account of work sponsored by an agency of the United States Government. Neither the United States Government nor any agency thereof, nor any of their employees, makes any warranty, express or implied, or assumes any legal liability or responsibility for the accuracy, completeness, or usefulness of any information, apparatus, product, or process disclosed, or represents that its use would not infringe privately owned rights. Reference herein to any specific commercial product, process, or service by trade name, trademark, manufacturer, or otherwise, does not necessarily constitute or imply its endorsement, recommendation, or favoring by the United States Government or any agency thereof. The views and opinions of authors expressed herein do not necessarily state or reflect those of the United States Government or any agency thereof. 


\title{
Data Compilation for AGR-3/4 Matrix Ring Blank Lot RDKRS
}

\author{
John D. Hunn, Michael P. Trammell, and Fred C. Montgomery \\ Oak Ridge National Laboratory
}

This document is a compilation of compacting and characterization data for the matrix ring blank lot RDKRS. These ring blanks were made using Hexion Durite SC-1008 resin mixed with natural and synthetic graphite flake. The $26 \mathrm{~mm}$ diameter, $63 \mathrm{~mm}$ long solid cylindrical ring blanks were shipped from Oak Ridge National Laboratory (ORNL) to Idaho National Laboratory (INL) for machining and insertion into the Advanced Gas Reactor Fuel Development and Qualification (AGR) program's third irradiation test (AGR-3/4).

In the AGR-3/4 irradiation experiment, each $12.3 \mathrm{~mm}$ diameter, $12.5 \mathrm{~mm}$ long cylindrical fuel compact will contain twenty designed-to-fail (DTF) particles distributed along the centerline of the compact. The DTF coating is a high density, high anisotropy pyrocarbon coating of nominal $20 \mu \mathrm{m}$ thickness that is deposited directly on the kernel. This coating is designed to fail early in the AGR-3/4 irradiation test, resulting in a controlled release of fission products which can be analyzed to provide data on fission product transport. The DTF will be surrounded by standard tristructural isotropic (TRISO) "driver fuel" particles. Information on the DTF and driver fuel particles can be found in ORNL/TM-2011-109 " Data Compilation for AGR-3/4 Designed-toFail (DTF) Fuel Particle Batch LEU03-07DTF " and ORNL/TM-2007/019, "Data Compilation for AGR-3/4 Driver Fuel Coated Particle Composite LEU03-09T." Information on the fuel compacts can be found in ORNL/TM-2011/124, "Data Compilation for AGR-3/4 Designed-toFail (DTF) Fuel Compact Lot (LEU03-10T-OP2/LEU03-07DTF-OP1)-Z"

Each irradiation test capsule will contain four compacts stacked in a single column. Matrix ring blanks will be machined with a center bore diameter $\sim 100-150 \mu \mathrm{m}$ larger than the diameter of the fuel compacts, so that the fuel stack can be placed inside the matrix ring. The outer diameter and length of matrix rings will also be machined. A graphite sleeve will be machined to surround the matrix ring. During irradiation, fission products will migrate out of the compact into these surrounding matrix and graphite rings.

The matrix ring blank lot, RDKRS, was determined to meet the product specifications for compact matrix ring blanks in section 5 of the AGR-3/4 DTF Fuel and Capsule Component Material Specifications (SPC-1214, Rev. 0). Table 1 provides a summary of key properties of the ring blanks.

Table 1. Summary of key ring blank properties in comparison to product specifications.

\begin{tabular}{|l|c|c|c|}
\hline \multicolumn{2}{|c|}{ Specified Parameters } & \multicolumn{2}{c|}{ RDKRS Ring Blanks } \\
\cline { 3 - 4 } & & mean & standard deviation \\
\hline Blank outer diameter $(\mathrm{mm})$ & $26.0 \pm 1$ & 26.01 & 0.03 \\
\hline Blank length $(\mathrm{mm})$ & $63.0 \pm 2$ & 62.9 & 0.2 \\
\hline Blank matrix density (g/cc) & $1.60 \pm 0.1$ & 1.621 & 0.010 \\
\hline Heavy metal contamination (ppmw U) & $\leq 0.5$ & 0.016 & 0.003 \\
\hline
\end{tabular}




\section{Table of Contents}

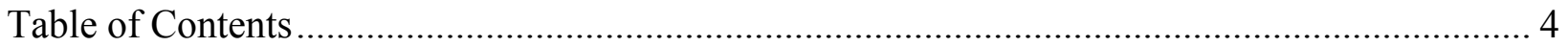

1 Material Identification Record for RDKRS Ring Blanks ......................................... 5

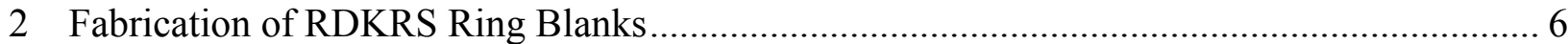

3 Impurity Analysis of Matrix, Resin, and Graphite .......................................................... 9

4 Characterization of RDKRS Ring Blanks.............................................................. 15

5 Additional Characterization of RDKRS Ring Blanks for Information Only...................... 26

6 Analysis of Ash Residue Remaining After Burn-Leach ............................................... 33

7 Analysis of Impurity Content in Machined Matrix Rings .............................................. 39

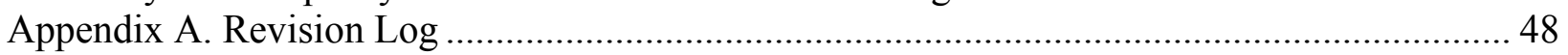




\section{Material Identification Record for RDKRS Ring Blanks}

Table 2 lists the materials used to make the RDKRS ring blanks, including intermediate batches and samples used for characterization. Thirty completed ring blanks were shipped to INL in February, 2011. Eight ring blanks were retained at ORNL and 12 ring blanks were consumed at ORNL by the QC acceptance testing. Table 3 lists the disposition of each ring blank.

Table 2. Material identification record for ring blank lot RDKRS

\begin{tabular}{|c|c|c|}
\hline Sample ID & Parent Material & Notes \\
\hline RD13371 & Asbury Graphite Mills & Natural graphite \\
\hline KRB2000 & SGL Carbon & Synthetic graphite \\
\hline $\mathrm{SC} 1008$ & Hexion & Durite resin lot LKOLA7410 \\
\hline RDKrS-0121211 & $\begin{array}{c}64 \mathrm{wt} \% \text { RD13371 } \\
16 \mathrm{wt} \% \text { KRB2000 } \\
20 w t \% \text { SC1008 }\end{array}$ & Graphite/resin blend \\
\hline RDKRS-G\#\#\# & RDKrS-0121211 & Ring blanks numbered G001 through G050 \\
\hline RDKRS-Z\#\#\# & RDKRS-G\#\#\# & $\begin{array}{l}\text { Ring blanks, numbered Z001 through Z050 } \\
\text { One to one correspondence to G\#\#\# recorded on } \\
\text { IRF20C (section 4) }\end{array}$ \\
\hline
\end{tabular}

Table 3. Disposition of RDKRS ring blanks

\begin{tabular}{|l|c|c|c|}
\hline \multicolumn{2}{|c|}{ Sent to INL } & Retained at ORNL & Consumed during analysis \\
\hline RDKRS-Z001 & RDKRS-Z024 & RDKRS-Z006 & RDKRS-Z003 \\
\hline RDKRS-Z002 & RDKRS-Z028 & RDKRS-Z008 & RDKRS-Z014 \\
\hline RDKRS-Z004 & RDKRS-Z029 & RDKRS-Z012 & RDKRS-Z015 \\
\hline RDKRS-Z005 & RDKRS-Z031 & RDKRS-Z016 & RDKRS-Z019 \\
\hline RDKRS-Z007 & RDKRS-Z032 & RDKRS-Z030 & RDKRS-Z025 \\
\hline RDKRS-Z009 & RDKRS-Z033 & RDKRS-Z038 & RDKRS-Z026 \\
\hline RDKRS-Z010 & RDKRS-Z034 & RDKRS-Z049 & RDKRS-Z027 \\
\hline RDKRS-Z011 & RDKRS-Z037 & RDKRS-Z050 & RDKRS-Z035 \\
\hline RDKRS-Z013 & RDKRS-Z039 & & RDKRS-Z036 \\
\hline RDKRS-Z017 & RDKRS-Z042 & & RDKRS-Z040 \\
\hline RDKRS-Z018 & RDKRS-Z043 & & RDKRS-Z041 \\
\hline RDKRS-Z020 & RDKRS-Z045 & & \\
\hline RDKRS-Z021 & RDKRS-Z046 & & \\
\hline RDKRS-Z022 & RDKRS-Z047 & & \\
\hline RDKRS-Z023 & RDKRS-Z048 & & \\
\hline
\end{tabular}




\section{Fabrication of RDKRS Ring Blanks}

Natural graphite (Asbury Graphite Mills RD13371), synthetic graphite (SGL Carbon KRB2000), and thermosetting resin (Hexion Durite SC1008-lot LKOLA7410) were combined in a weight ratio of 64:16:20 to make a matrix precursor graphite/resin blend. The blend was solvated with ethyl alcohol, mixed thoroughly, poured into pans, and allowed to dry. After drying, the caked powder was pulverized using a Holmes pulverizer. This process produced resin-coated (i.e., resinated) graphite particles. In order to ensure uniformity, one single batch of the graphite/resin blend (RDKrS-0121211) was produced for the fabrication of all the RDKRS ring blanks.

Compacting charges of the graphite/resin blend were weighed out and labeled RDKRS-G001 through G050. The compacting charges were formed into "green" ring blanks using a heated, single acting die and a manually operated Carver hydraulic press. Compacting was performed in accordance with AGR-COMP-SOP-11R0, "Standard Operating Procedures for Production of AGR 3/4 Matrix Ring Blanks." The compacting die, top punch, and bottom punch were heated to $50^{\circ} \mathrm{C}$. With all parts at temperature and the bottom punch in place, the pre-measured compacting charge was poured into the die. The top punch was then inserted and compaction was initiated. A dial gauge was used to monitor the distance between the press platens during compaction. A target distance was predetermined during compacting development, which would yield ring blanks of the desired length after shrinkage during carbonization of the resin. Once this target distance was reached, pressing was halted and the ring blank was held in the die for an additional 5 minutes before ejecting from the die. The maximum pressure required to compact the ring blanks ranged from 9-15 $\mathrm{MPa}$. The specific maximum pressure applied to each ring blank is recorded in Table 4. In total, 50 green ring blanks were fabricated. The green ring blanks retained the material designation of RDKRS-G001 through G050.

All 50 green ring blanks were carbonized and heat treated according to AGR-COMP-SOP-04R0, "Standard Operating Procedure for Carbonizing Compacts," and AGR-COMP-SOP-05R1, "Standard Operating Procedure for Heat-treating Compacts." Table 4 shows the process conditions used. Green ring blanks were slowly heated at $2^{\circ} \mathrm{C} / \mathrm{min}$ to $950^{\circ} \mathrm{C}$ in flowing helium atmosphere and then held at $950^{\circ} \mathrm{C}$ for 1 hour. The heating rate was less than used for smaller diameter AGR fuel compacts $\left(\sim 5^{\circ} \mathrm{C} / \mathrm{min}\right)$, because extra time was needed to allow evolved gases to diffuse out of the larger ring blank cylinders and prevent cracking. The carbonization furnace was allowed to cool to room temperature under no power before removing ring blanks for transfer to the heat-treatment furnace. Heat-treatment was performed under vacuum after backfilling with argon three times. Using the standard AGR compact heat-treatment process, carbonized ring blanks were heated at $20^{\circ} \mathrm{C} / \mathrm{min}$ to $1800^{\circ} \mathrm{C}$, held for 1 hour, cooled at $<20^{\circ} \mathrm{C} / \mathrm{min}$ to $700^{\circ} \mathrm{C}$, then cooled under no power to room temperature before removing.

After heat-treatment, all 50 ring blanks were selected from RDKRS-G001 through G050 for use. As instructed in AGR-CHAR-PIP-20R1, "Product Inspection Plan for AGR-3/4 Ring Blank Lots," these 50 ring blanks were randomized and relabeled as RDKRS-Z001 through Z050. A record of the original G-number for each Z-numbered ring blank can be found in inspection report form IRF-20C (section 4). After relabeling, the ring blanks were characterized for product acceptance according to product inspection plan PIP-20. This plan calls for measurement of ring blank length, diameter, mass, matrix density, uranium content and impurity content. 
ORNL/TM-2011/127

Table 4. Summary of process conditions used in making RDKRS-Z ring blanks

\begin{tabular}{|c|c|c|c|c|c|c|c|c|c|}
\hline \multirow[b]{2}{*}{ Ring blank ID } & \multicolumn{5}{|c|}{ Compacting and carbonization parameters } & \multicolumn{4}{|c|}{ Heat-treatment parameters } \\
\hline & $\begin{array}{c}\text { Max. Pressure } \\
(\mathrm{MPa})\end{array}$ & $\begin{array}{c}\text { Heating Rate } \\
\left({ }^{\circ} \mathrm{C} / \mathrm{min}\right)\end{array}$ & $\begin{array}{c}\text { Max. Temp. } \\
\left({ }^{\circ} \mathrm{C}\right)\end{array}$ & $\begin{array}{c}\text { Hold Time } \\
\text { (hr) }\end{array}$ & Atmosphere & $\begin{array}{l}\text { Heating Rate } \\
\left({ }^{\circ} \mathrm{C} / \mathrm{min}\right)\end{array}$ & $\begin{array}{l}\text { Max. Temp. } \\
\left({ }^{\circ} \mathrm{C}\right)\end{array}$ & $\begin{array}{l}\text { Hold Time } \\
\text { (hr) }\end{array}$ & Atmosphere \\
\hline RDKRS-Z001 & 10.7 & 2 & 950 & 1 & flowing $\mathrm{He}$ & 20 & 1800 & 1 & vacuum \\
\hline RDKRS-Z002 & 11.3 & 2 & 950 & 1 & flowing $\mathrm{He}$ & 20 & 1800 & 1 & vacuum \\
\hline RDKRS-Z003 & 12.1 & 2 & 950 & 1 & flowing $\mathrm{He}$ & 20 & 1800 & 1 & vacuum \\
\hline RDKRS-Z004 & 12.7 & 2 & 950 & 1 & flowing $\mathrm{He}$ & 20 & 1800 & 1 & vacuum \\
\hline RDKRS-Z005 & 9.9 & 2 & 950 & 1 & flowing $\mathrm{He}$ & 20 & 1800 & 1 & vacuum \\
\hline RDKRS-Z006 & 12.5 & 2 & 950 & 1 & flowing $\mathrm{He}$ & 20 & 1800 & 1 & vacuum \\
\hline RDKRS-Z007 & 9.9 & 2 & 950 & 1 & flowing $\mathrm{He}$ & 20 & 1800 & 1 & vacuum \\
\hline RDKRS-Z008 & 10.0 & 2 & 950 & 1 & flowing $\mathrm{He}$ & 20 & 1800 & 1 & vacuum \\
\hline RDKRS-Z009 & 9.8 & 2 & 950 & 1 & flowing $\mathrm{He}$ & 20 & 1800 & 1 & vacuum \\
\hline RDKRS-Z010 & 14.3 & 2 & 950 & 1 & flowing $\mathrm{He}$ & 20 & 1800 & 1 & vacuum \\
\hline RDKRS-Z011 & 10.0 & 2 & 950 & 1 & flowing $\mathrm{He}$ & 20 & 1800 & 1 & vacuum \\
\hline RDKRS-Z012 & 9.9 & 2 & 950 & 1 & flowing $\mathrm{He}$ & 20 & 1800 & 1 & vacuum \\
\hline RDKRS-Z013 & 9.2 & 2 & 950 & 1 & flowing $\mathrm{He}$ & 20 & 1800 & 1 & vacuum \\
\hline RDKRS-Z014 & 12.2 & 2 & 950 & 1 & flowing $\mathrm{He}$ & 20 & 1800 & 1 & vacuum \\
\hline RDKRS-Z015 & 11.2 & 2 & 950 & 1 & flowing $\mathrm{He}$ & 20 & 1800 & 1 & vacuum \\
\hline RDKRS-Z016 & 9.5 & 2 & 950 & 1 & flowing $\mathrm{He}$ & 20 & 1800 & 1 & vacuum \\
\hline RDKRS-Z017 & 9.8 & 2 & 950 & 1 & flowing $\mathrm{He}$ & 20 & 1800 & 1 & vacuum \\
\hline RDKRS-Z018 & 9.6 & 2 & 950 & 1 & flowing $\mathrm{He}$ & 20 & 1800 & 1 & vacuum \\
\hline RDKRS-Z019 & 10.0 & 2 & 950 & 1 & flowing $\mathrm{He}$ & 20 & 1800 & 1 & vacuum \\
\hline RDKRS-Z020 & 9.8 & 2 & 950 & 1 & flowing $\mathrm{He}$ & 20 & 1800 & 1 & vacuum \\
\hline RDKRS-Z021 & 12.4 & 2 & 950 & 1 & flowing $\mathrm{He}$ & 20 & 1800 & 1 & vacuum \\
\hline RDKRS-Z022 & 9.9 & 2 & 950 & 1 & flowing $\mathrm{He}$ & 20 & 1800 & 1 & vacuum \\
\hline RDKRS-Z023 & 10.4 & 2 & 950 & 1 & flowing $\mathrm{He}$ & 20 & 1800 & 1 & vacuum \\
\hline RDKRS-Z024 & 10.3 & 2 & 950 & 1 & flowing $\mathrm{He}$ & 20 & 1800 & 1 & vacuum \\
\hline RDKRS-Z025 & 9.2 & 2 & 950 & 1 & flowing $\mathrm{He}$ & 20 & 1800 & 1 & vacuum \\
\hline
\end{tabular}

Task Manager Review Micharl drane \&

QAS Review
Date

$5 / 17 / 11$

Date

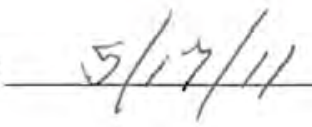


Table 4 (continued). Summary of process conditions used in making RDKRS-Z ring blanks

\begin{tabular}{|c|c|c|c|c|c|c|c|c|c|}
\hline \multirow[b]{2}{*}{ Ring blank ID } & \multicolumn{5}{|c|}{ Compacting and carbonization parameters } & \multicolumn{4}{|c|}{ Heat-treatment parameters } \\
\hline & $\begin{array}{c}\text { Max. Pressure } \\
(\mathrm{MPa})\end{array}$ & $\begin{array}{c}\text { Heating Rate } \\
\left({ }^{\circ} \mathrm{C} / \mathrm{min}\right)\end{array}$ & $\begin{array}{l}\text { Max. Temp. } \\
\left({ }^{\circ} \mathrm{C}\right)\end{array}$ & $\begin{array}{l}\text { Hold Time } \\
(\mathrm{hr})\end{array}$ & Atmosphere & $\begin{array}{l}\text { Heating Rate } \\
\left({ }^{\circ} \mathrm{C} / \mathrm{min}\right)\end{array}$ & $\begin{array}{l}\text { Max. Temp. } \\
\left({ }^{\circ} \mathrm{C}\right)\end{array}$ & $\begin{array}{l}\text { Hold Time } \\
(\mathrm{hr})\end{array}$ & Atmosphere \\
\hline RDKRS-Z026 & 10.7 & 2 & 950 & 1 & flowing $\mathrm{He}$ & 20 & 1800 & 1 & vacuum \\
\hline RDKRS-Z027 & 12.2 & 2 & 950 & 1 & flowing $\mathrm{He}$ & 20 & 1800 & 1 & vacuum \\
\hline RDKRS-Z028 & 13.3 & 2 & 950 & 1 & flowing $\mathrm{He}$ & 20 & 1800 & 1 & vacuum \\
\hline RDKRS-Z029 & 9.9 & 2 & 950 & 1 & flowing $\mathrm{He}$ & 20 & 1800 & 1 & vacuum \\
\hline RDKRS-Z030 & 9.4 & 2 & 950 & 1 & flowing $\mathrm{He}$ & 20 & 1800 & 1 & vacuum \\
\hline RDKRS-Z031 & 11.4 & 2 & 950 & 1 & flowing $\mathrm{He}$ & 20 & 1800 & 1 & vacuum \\
\hline RDKRS-Z032 & 12.3 & 2 & 950 & 1 & flowing $\mathrm{He}$ & 20 & 1800 & 1 & vacuum \\
\hline RDKRS-Z033 & 9.0 & 2 & 950 & 1 & flowing $\mathrm{He}$ & 20 & 1800 & 1 & vacuum \\
\hline RDKRS-Z034 & 13.7 & 2 & 950 & 1 & flowing $\mathrm{He}$ & 20 & 1800 & 1 & vacuum \\
\hline RDKRS-Z035 & 13.9 & 2 & 950 & 1 & flowing $\mathrm{He}$ & 20 & 1800 & 1 & vacuum \\
\hline RDKRS-Z036 & 12.6 & 2 & 950 & 1 & flowing $\mathrm{He}$ & 20 & 1800 & 1 & vacuum \\
\hline RDKRS-Z037 & 9.5 & 2 & 950 & 1 & flowing $\mathrm{He}$ & 20 & 1800 & 1 & vacuum \\
\hline RDKRS-Z038 & 12.2 & 2 & 950 & 1 & flowing $\mathrm{He}$ & 20 & 1800 & 1 & vacuum \\
\hline RDKRS-Z039 & 13.2 & 2 & 950 & 1 & flowing $\mathrm{He}$ & 20 & 1800 & 1 & vacuum \\
\hline RDKRS-Z040 & 9.6 & 2 & 950 & 1 & flowing $\mathrm{He}$ & 20 & 1800 & 1 & vacuum \\
\hline RDKRS-Z041 & 13.1 & 2 & 950 & 1 & flowing $\mathrm{He}$ & 20 & 1800 & 1 & vacuum \\
\hline RDKRS-Z042 & 10.0 & 2 & 950 & 1 & flowing $\mathrm{He}$ & 20 & 1800 & 1 & vacuum \\
\hline RDKRS-Z043 & 10.9 & 2 & 950 & 1 & flowing $\mathrm{He}$ & 20 & 1800 & 1 & vacuum \\
\hline RDKRS-Z044 & 9.5 & 2 & 950 & 1 & flowing $\mathrm{He}$ & 20 & 1800 & 1 & vacuum \\
\hline RDKRS-Z045 & 11.6 & 2 & 950 & 1 & flowing $\mathrm{He}$ & 20 & 1800 & 1 & vacuum \\
\hline RDKRS-Z046 & 10.2 & 2 & 950 & 1 & flowing $\mathrm{He}$ & 20 & 1800 & 1 & vacuum \\
\hline RDKRS-Z047 & 9.9 & 2 & 950 & 1 & flowing $\mathrm{He}$ & 20 & 1800 & 1 & vacuum \\
\hline RDKRS-Z048 & 9.5 & 2 & 950 & 1 & flowing $\mathrm{He}$ & 20 & 1800 & 1 & vacuum \\
\hline RDKRS-Z049 & 9.8 & 2 & 950 & 1 & flowing $\mathrm{He}$ & 20 & 1800 & 1 & vacuum \\
\hline RDKRS-Z050 & 10.0 & 2 & 950 & 1 & flowing $\mathrm{He}$ & 20 & 1800 & 1 & vacuum \\
\hline
\end{tabular}




\section{Impurity Analysis of Matrix, Resin, and Graphite}

The AGR-3/4 Fuel Specification (SPC-1214, Rev. 0) specifies maximum limits on the elemental impurities $\mathrm{Al}, \mathrm{Ca}, \mathrm{Ti}+\mathrm{V}, \mathrm{Cr}, \mathrm{Mn}, \mathrm{Fe}, \mathrm{Co}, \mathrm{Ni}$, and $\mathrm{U}$. The natural graphite, synthetic graphite, and thermosetting resin used to make the matrix often contain measurable amounts of these impurities. It is beneficial if the graphite and resin have low concentrations of the impurities of interest to help ensure that the ring blanks made from the graphite/resin blend will be within specification. Part of the compacting development effort was selection of the natural graphite, synthetic graphite, and resin. One criterion for the acceptability of the graphite or resin was if it could produce a ring blank that was within specification on impurities. The selection process included receiving natural graphite and synthetic graphite and testing them via glow discharge mass spectrometry (GDMS) in order to establish their initial impurity concentrations. In addition to the as-received graphite samples, both the resin alone and the graphite/resin blend were carbonized and the carbonized powder was also measured by GDMS.

Table 5 is a summary of the as-received impurity levels for the natural graphite and synthetic graphite that were used to make the RDKRS ring blanks, as well as the impurity levels for the carbonized samples of the resin and the RDKrS-0121211 graphite/resin blend. All the specified impurities for all the components were below the specified limits.

Table 5. Matrix constituents that were used in AGR-3/4 RDKRS ring blanks

\begin{tabular}{|c||c|c|c|c|c|}
\hline \multirow{2}{*}{ Element } & \multicolumn{5}{|c|}{ Impurity concentration (ppm-wt) } \\
\cline { 2 - 6 } & Specification & $\begin{array}{c}\text { Natural Graphite } \\
\text { RD13371 }\end{array}$ & $\begin{array}{c}\text { Synthetic Graphite } \\
\text { KRB2000 }\end{array}$ & $\begin{array}{c}\text { Carbonized Resin } \\
\text { Durite SC-1008 }\end{array}$ & $\begin{array}{c}\text { Carbonized Matrix } \\
\text { RDKrS-0121211 }\end{array}$ \\
\hline \hline $\mathrm{Al}$ & $\leq 20$ & 8.3 & 0.13 & 3.3 & 4.3 \\
\hline $\mathrm{Ca}$ & $\leq 45$ & 10 & 0.31 & 3.1 & 4.8 \\
\hline $\mathrm{Ti}+\mathrm{V}$ & $\leq 85$ & 1.01 & $<0.07$ & 0.14 & 0.29 \\
\hline $\mathrm{Cr}$ & $\leq 10$ & $<0.5$ & $<0.5$ & $<0.5$ & $<0.5$ \\
\hline $\mathrm{Mn}$ & $\leq 10$ & 0.29 & $<0.05$ & 0.12 & 0.35 \\
\hline $\mathrm{Fe}$ & $\leq 20$ & 13 & 0.11 & 0.87 & 12 \\
\hline $\mathrm{Co}$ & $\leq 10$ & $<0.05$ & $<0.05$ & $<0.05$ & $<0.05$ \\
\hline $\mathrm{Ni}$ & $\leq 10$ & 1.4 & 0.12 & 0.33 & $<.18$ \\
\hline $\mathrm{U}$ & $\leq 0.5$ & $<0.05$ & $<0.05$ & $<0.05$ & $<0.05$ \\
\hline
\end{tabular}

The following pages show the impurity analysis reports for the natural graphite, synthetic graphite, resin, and matrix/resin blend samples listed in Table 5. Also attached is an additional certificate of analysis for the resin. Note that an expiration date of 6 months from the manufacture date was set for producing compacts from the resin. The resin manufacture date was 12/04/10 and RDKRS compacting was completed on 2/08/11. 


$\begin{array}{llll}\text { Customer: } & \text { UT-Battelle Oak Ridge } & \text { P.O.\# } & \text { CC } \\ & \text { 1 Bethel Valley Rd, Oak Ridge, TN 37823-6063 USA } & & \\ \text { Date: } & \text { 24-Mar-11 } & \text { Job \# } & \text { S0BCT802 } \\ \text { Customer ID: } \text { C } \text { powder } & \text { Sample ID: } & \text { S110317086 }\end{array}$

\section{RD\# 13371}

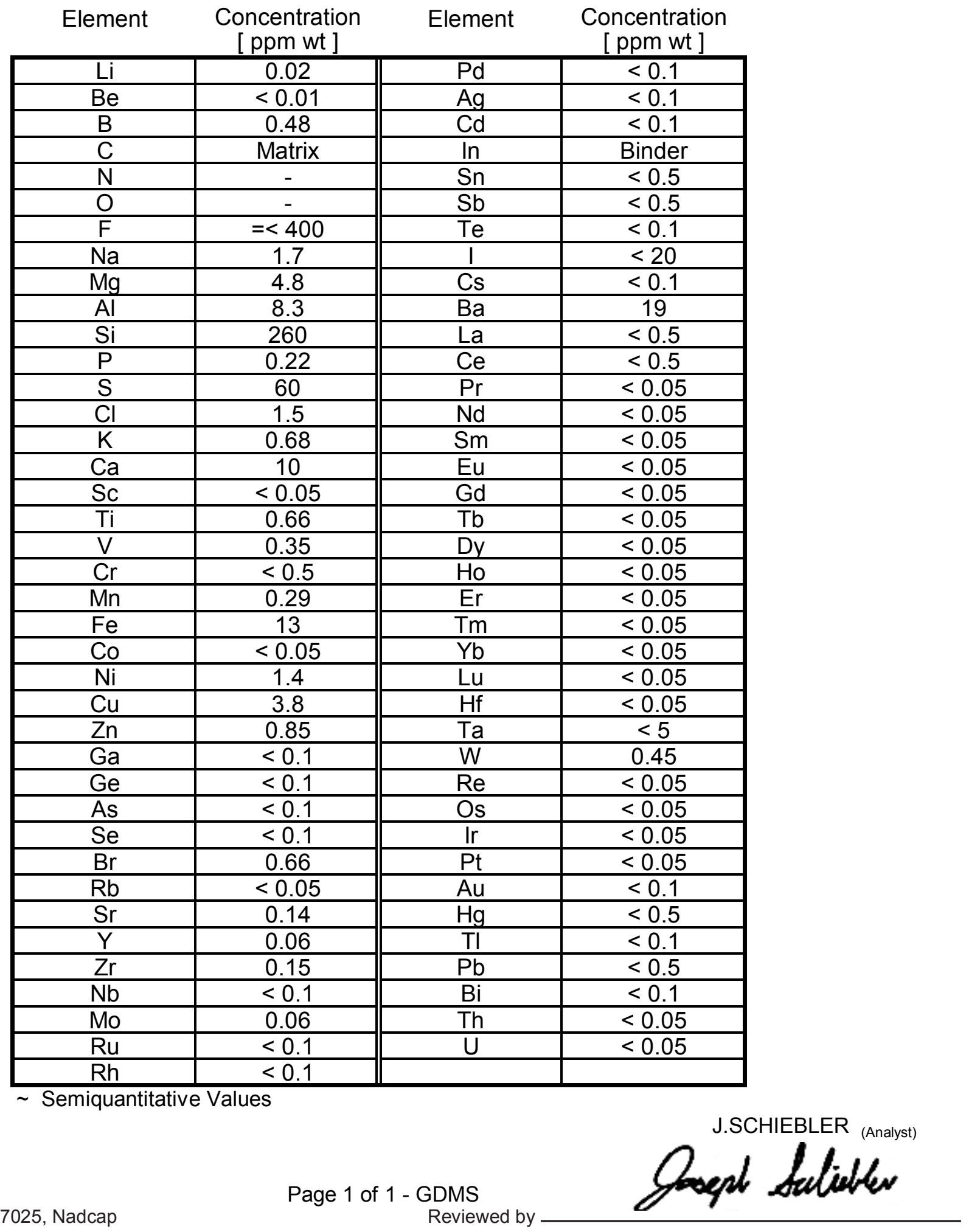




$\begin{array}{llll}\text { Customer: } & \text { UT-Battelle Oak Ridge } & \text { P.O.\# } & \text { CC } \\ & \text { 1 Bethel Valley Rd, Oak Ridge, TN 37823-6063 USA } & & \\ \text { Date: } & \text { 24-Mar-11 } & \text { Job \# } & \text { S0BCT802 } \\ \text { Customer ID: C powder } & \text { Sample ID: } & \text { S110317087 } \\ & \text { KRB 2000 } & & \end{array}$

\begin{tabular}{|c|c|c|c|}
\hline Element & $\begin{array}{c}\text { Concentration } \\
\text { [ ppm wt ] }\end{array}$ & Element & $\begin{array}{c}\text { Concentration } \\
\text { [ppm wt ] }\end{array}$ \\
\hline $\mathrm{Li}$ & $<0.01$ & $\mathrm{Pd}$ & $<0.1$ \\
\hline $\mathrm{Be}$ & $<0.01$ & $\mathrm{Ag}$ & $<0.1$ \\
\hline $\mathrm{B}$ & 0.19 & $\mathrm{Cd}$ & $<0.1$ \\
\hline C & Matrix & $\ln$ & Binder \\
\hline $\mathrm{N}$ & - & Sn & $<0.5$ \\
\hline $\mathrm{O}$ & - & $\mathrm{Sb}$ & $<0.5$ \\
\hline $\mathrm{F}$ & $=<10$ & $\mathrm{Te}$ & $<0.1$ \\
\hline $\mathrm{Na}$ & 0.92 & 1 & $<20$ \\
\hline $\mathrm{Mg}$ & $<0.5$ & Cs & $<0.1$ \\
\hline $\mathrm{Al}$ & 0.13 & $\mathrm{Ba}$ & $<0.1$ \\
\hline Si & 3.1 & $\mathrm{La}$ & $<0.5$ \\
\hline $\mathrm{P}$ & $<0.1$ & $\mathrm{Ce}$ & $<0.5$ \\
\hline$S$ & 5.4 & $\mathrm{Pr}$ & $<0.05$ \\
\hline $\mathrm{Cl}$ & 9.3 & $\mathrm{Nd}$ & $<0.05$ \\
\hline $\mathrm{K}$ & $<0.1$ & $\mathrm{Sm}$ & $<0.05$ \\
\hline $\mathrm{Ca}$ & 0.31 & $\mathrm{Eu}$ & $<0.05$ \\
\hline$\overline{S c}$ & $<0.05$ & $\mathrm{Gd}$ & $<0.05$ \\
\hline $\mathrm{Ti}$ & 0.06 & $\mathrm{~Tb}$ & $<0.05$ \\
\hline $\mathrm{V}$ & $<0.01$ & Dy & $<0.05$ \\
\hline $\mathrm{Cr}$ & $<0.5$ & $\mathrm{Ho}$ & $<0.05$ \\
\hline $\mathrm{Mn}$ & $<0.05$ & $\mathrm{Er}$ & $<0.05$ \\
\hline $\mathrm{Fe}$ & 0.11 & $\mathrm{Tm}$ & $<0.05$ \\
\hline Co & $<0.05$ & $\mathrm{Yb}$ & $<0.05$ \\
\hline $\mathrm{Ni}$ & 0.12 & $\mathrm{Lu}$ & $<0.05$ \\
\hline $\mathrm{Cu}$ & $<0.1$ & $\mathrm{Hf}$ & $<0.05$ \\
\hline$\overline{Z n}$ & $<0.1$ & $\mathrm{Ta}$ & $<5$ \\
\hline $\mathrm{Ga}$ & $<0.1$ & W & 0.09 \\
\hline $\mathrm{Ge}$ & $<0.1$ & $\mathrm{Re}$ & $<0.05$ \\
\hline As & $<0.1$ & Os & $<0.05$ \\
\hline $\mathrm{Se}$ & $<0.1$ & $\mathrm{Ir}$ & $<0.05$ \\
\hline $\mathrm{Br}$ & $<0.1$ & $\mathrm{Pt}$ & $<0.05$ \\
\hline $\mathrm{Rb}$ & $<0.05$ & $\mathrm{Au}$ & $<0.1$ \\
\hline $\mathrm{Sr}$ & $<0.05$ & $\mathrm{Hg}$ & $<0.5$ \\
\hline $\bar{Y}$ & $<0.05$ & $\mathrm{TI}$ & $<0.1$ \\
\hline $\mathrm{Zr}$ & $<0.05$ & $\mathrm{~Pb}$ & $<0.5$ \\
\hline $\mathrm{Nb}$ & $<0.1$ & $\mathrm{Bi}$ & $<0.1$ \\
\hline Mo & $<0.05$ & Th & $<0.05$ \\
\hline $\mathrm{Ru}$ & $<0.1$ & $\mathrm{U}$ & $<0.05$ \\
\hline $\mathrm{Rh}$ & $<0.1$ & & \\
\hline
\end{tabular}

$\sim$ Semiquantitative Values 


$\begin{array}{llll}\text { Customer: } & \begin{array}{l}\text { UT-Battelle Oak Ridge } \\ \text { 1 Bethel Valley Rd, Oak Ridge, TN 37823-6063 USA }\end{array} & \text { P.O.\# } & \text { CC } \\ \text { Date: } & \text { 24-Mar-11 } & \text { Job\# } & \text { S0BCT802 } \\ \text { Customer ID: } \text { C powder } & \text { Sample ID: } & \text { S110317088 } \\ & \text { Durite SC-1008 } & & \end{array}$

\begin{tabular}{|c|c|c|c|}
\hline Element & $\begin{array}{c}\text { Concentration } \\
\text { [ ppm wt ] }\end{array}$ & Element & $\begin{array}{c}\text { Concentration } \\
\text { [ppm wt ] }\end{array}$ \\
\hline $\mathrm{Li}$ & 0.42 & $\mathrm{Pd}$ & $<0.1$ \\
\hline $\mathrm{Be}$ & $<0.01$ & $\mathrm{Ag}$ & $<0.1$ \\
\hline $\mathrm{B}$ & 0.65 & $\mathrm{Cd}$ & $<0.1$ \\
\hline C & Matrix & $\ln$ & Binder \\
\hline $\mathrm{N}$ & - & Sn & $<0.5$ \\
\hline $\mathrm{O}$ & - & $\mathrm{Sb}$ & $<0.5$ \\
\hline $\mathrm{F}$ & $=<100$ & $\mathrm{Te}$ & $<0.1$ \\
\hline $\mathrm{Na}$ & 13 & I & $=<30$ \\
\hline $\mathrm{Mg}$ & 0.84 & Cs & $<0.1$ \\
\hline $\mathrm{Al}$ & 3.3 & $\mathrm{Ba}$ & 0.15 \\
\hline Si & 14 & $\mathrm{La}$ & $=<1$ \\
\hline $\mathrm{P}$ & 0.69 & $\mathrm{Ce}$ & $<0.5$ \\
\hline$S$ & 2.6 & $\mathrm{Pr}$ & $<0.05$ \\
\hline $\mathrm{Cl}$ & 0.37 & $\mathrm{Nd}$ & $<0.05$ \\
\hline $\mathrm{K}$ & 0.35 & $\mathrm{Sm}$ & $<0.05$ \\
\hline $\mathrm{Ca}$ & 3.1 & $\mathrm{Eu}$ & $<0.05$ \\
\hline$\overline{S c}$ & $<0.05$ & $\mathrm{Gd}$ & $<0.05$ \\
\hline $\mathrm{Ti}$ & 0.07 & $\mathrm{~Tb}$ & $<0.05$ \\
\hline $\mathrm{V}$ & 0.07 & Dy & $<0.05$ \\
\hline $\mathrm{Cr}$ & $<0.5$ & $\mathrm{Ho}$ & $<0.05$ \\
\hline $\mathrm{Mn}$ & 0.12 & $\mathrm{Er}$ & $<0.05$ \\
\hline $\mathrm{Fe}$ & 0.87 & $\mathrm{Tm}$ & $<0.05$ \\
\hline Co & $<0.05$ & $\mathrm{Yb}$ & $<0.05$ \\
\hline $\mathrm{Ni}$ & 0.33 & $\mathrm{Lu}$ & $<0.05$ \\
\hline $\mathrm{Cu}$ & 0.37 & $\mathrm{Hf}$ & $<0.05$ \\
\hline$\overline{Z n}$ & $<0.1$ & $\mathrm{Ta}$ & $<5$ \\
\hline $\mathrm{Ga}$ & $<0.1$ & W & 0.13 \\
\hline $\mathrm{Ge}$ & $<0.1$ & $\mathrm{Re}$ & $<0.05$ \\
\hline As & $<0.1$ & Os & $<0.05$ \\
\hline $\mathrm{Se}$ & $<0.1$ & $\mathrm{Ir}$ & $<0.05$ \\
\hline $\mathrm{Br}$ & $<0.1$ & $\mathrm{Pt}$ & $<0.05$ \\
\hline $\mathrm{Rb}$ & $<0.05$ & $\mathrm{Au}$ & $<0.1$ \\
\hline $\mathrm{Sr}$ & $<0.05$ & $\mathrm{Hg}$ & $<0.5$ \\
\hline $\bar{Y}$ & $<0.05$ & $\mathrm{TI}$ & $<0.1$ \\
\hline $\mathrm{Zr}$ & 0.1 & $\mathrm{~Pb}$ & $<0.5$ \\
\hline $\mathrm{Nb}$ & $<0.1$ & $\mathrm{Bi}$ & $<0.1$ \\
\hline Mo & $<0.05$ & Th & $<0.05$ \\
\hline $\mathrm{Ru}$ & $<0.1$ & $\mathrm{U}$ & $<0.05$ \\
\hline $\mathrm{Rh}$ & $<0.1$ & & \\
\hline
\end{tabular}

$\sim$ Semiquantitative Values 


$\begin{array}{llll}\text { Customer: } & \text { UT-Battelle Oak Ridge } & \text { P.O.\# } & \text { CC } \\ & \text { 1 Bethel Valley Rd, Oak Ridge, TN 37823-6063 USA } & & \\ \text { Date: } & \text { 24-Mar-11 } & \text { Job\# } & \text { S0BCT802 } \\ \text { Customer ID: } \text { C powder } & \text { Sample ID: } & \text { S110317089 } \\ & \text { RDKRS 0121211 } & & \end{array}$

\begin{tabular}{|c|c|c|c|}
\hline Element & $\begin{array}{c}\text { Concentration } \\
\text { [ ppm wt ] }\end{array}$ & Element & $\begin{array}{c}\text { Concentration } \\
\text { [ppm wt ] }\end{array}$ \\
\hline $\mathrm{Li}$ & 0.05 & $\mathrm{Pd}$ & $<0.1$ \\
\hline $\mathrm{Be}$ & $<0.01$ & $\mathrm{Ag}$ & $<0.1$ \\
\hline $\mathrm{B}$ & 0.32 & $\mathrm{Cd}$ & $<0.1$ \\
\hline C & Matrix & $\ln$ & Binder \\
\hline $\mathrm{N}$ & - & Sn & $<0.5$ \\
\hline $\mathrm{O}$ & - & $\mathrm{Sb}$ & $<0.5$ \\
\hline $\mathrm{F}$ & $<5$ & $\mathrm{Te}$ & $<0.1$ \\
\hline $\mathrm{Na}$ & 0.82 & I & $<20$ \\
\hline $\mathrm{Mg}$ & 3.5 & Cs & $<0.1$ \\
\hline $\mathrm{Al}$ & 4.3 & $\mathrm{Ba}$ & 2.9 \\
\hline Si & 190 & $\mathrm{La}$ & $<0.5$ \\
\hline $\mathrm{P}$ & 0.42 & $\mathrm{Ce}$ & $<0.5$ \\
\hline$S$ & 30 & $\mathrm{Pr}$ & $<0.05$ \\
\hline $\mathrm{Cl}$ & 0.89 & $\mathrm{Nd}$ & $<0.05$ \\
\hline $\mathrm{K}$ & $<0.1$ & $\mathrm{Sm}$ & $<0.05$ \\
\hline $\mathrm{Ca}$ & 4.8 & $\mathrm{Eu}$ & $<0.05$ \\
\hline$\overline{S c}$ & $<0.05$ & $\mathrm{Gd}$ & $<0.05$ \\
\hline $\mathrm{Ti}$ & 0.27 & $\mathrm{~Tb}$ & $<0.05$ \\
\hline $\mathrm{V}$ & 0.02 & Dy & $<0.05$ \\
\hline $\mathrm{Cr}$ & $<0.5$ & $\mathrm{Ho}$ & $<0.05$ \\
\hline $\mathrm{Mn}$ & 0.35 & $\mathrm{Er}$ & $<0.05$ \\
\hline $\mathrm{Fe}$ & 12 & $\mathrm{Tm}$ & $<0.05$ \\
\hline Co & $<0.05$ & $\mathrm{Yb}$ & $<0.05$ \\
\hline $\mathrm{Ni}$ & 0.18 & $\mathrm{Lu}$ & $<0.05$ \\
\hline $\mathrm{Cu}$ & 0.28 & $\mathrm{Hf}$ & $<0.05$ \\
\hline$\overline{Z n}$ & $<0.1$ & $\mathrm{Ta}$ & $<5$ \\
\hline $\mathrm{Ga}$ & $<0.1$ & W & 0.18 \\
\hline $\mathrm{Ge}$ & $<0.1$ & $\mathrm{Re}$ & $<0.05$ \\
\hline As & $<0.1$ & Os & $<0.05$ \\
\hline $\mathrm{Se}$ & $<0.1$ & $\mathrm{Ir}$ & $<0.05$ \\
\hline $\mathrm{Br}$ & $<0.1$ & $\mathrm{Pt}$ & $<0.05$ \\
\hline $\mathrm{Rb}$ & $<0.05$ & $\mathrm{Au}$ & $<0.1$ \\
\hline $\mathrm{Sr}$ & $<0.05$ & $\mathrm{Hg}$ & $<0.5$ \\
\hline $\bar{Y}$ & $<0.05$ & $\mathrm{TI}$ & $<0.1$ \\
\hline $\mathrm{Zr}$ & $<0.05$ & $\mathrm{~Pb}$ & $<0.5$ \\
\hline $\mathrm{Nb}$ & $<0.1$ & $\mathrm{Bi}$ & $<0.1$ \\
\hline Mo & 0.05 & Th & $<0.05$ \\
\hline $\mathrm{Ru}$ & $<0.1$ & $\mathrm{U}$ & $<0.05$ \\
\hline $\mathrm{Rh}$ & $<0.1$ & & \\
\hline
\end{tabular}

$\sim$ Semiquantitative Values 


\section{Momentive Specialty Chemicals Inc. Certificate of Analysis}

Customer \#: 32710

Customer Address:

CAPITAL RESIN CORPORATION

324 DERING AVENUE

COLUMBUS DH 43207

USA

$\begin{array}{ll}\text { Ship Date: } & 12 / 06 / 2010 \\ \text { DDN: } & 83099081 \\ \text { Customer - PO\#: } & 901723 \\ \text { Date of MFG: } & 12 / 04 / 2010\end{array}$

$\begin{array}{ll}\text { Product Description: } & \text { Durite(TM) SC-1008 } \\ & \text { DS3271/450\# }\end{array}$ $\begin{array}{ll}\text { Product Description: } & \text { Durite(TM) SC } \\ & \text { DS3271/450\# }\end{array}$
KAY FREY

614-445-7177

$614-445-7290$

305922

Property Value Units Specification Ranges Test Method

Lot Number: I.KOLA7410

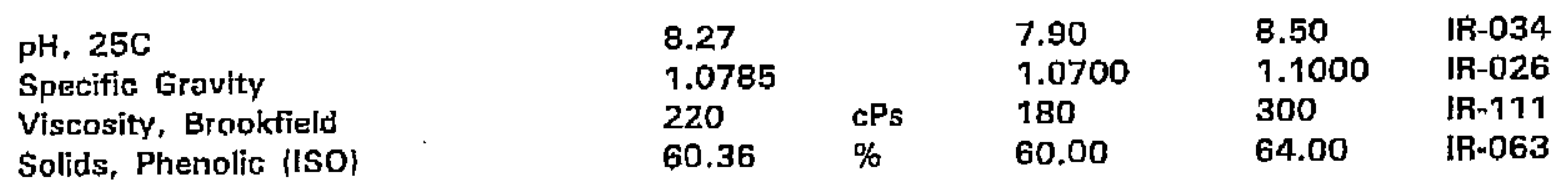

CERTIFICATE OF COMPLIANCE

It is hercby gertified that Hexion's Phenolic Hesin, SC-100B,

shipper in this lot has been produced in accordence with Mllitery specificotion laesin, Plenolic, Laminatingl MlL-F9299C, Grades A and B, deter Dacember 3, 1968. It is recummended that SC-1008 be stored in a cool place. Storage life is materially incrensed by retrigerazed storage. SC-1008 has a useble life of one month at 70 तegrage $F$ and six munths at 40 degrcos $F$.

Jeff A LaDuke

Quality Assurance

Aп 1S09001:2000 Certified Compeny

GHIPPED FRDM: Momentive. Specialty Chemicals 6200 Campground Road Louisville, KY 40216 Phone: 502-449-6563 


\section{Characterization of RDKRS Ring Blanks}

This section contains characterization data for the RDKRS ring blanks. The data was obtained according to product inspection plan AGR-CHAR-PIP-20R1, "Product Inspection Plan for AGR3/4 Matrix Ring Blank Lots." The matrix ring blank lot, RDKRS, was determined to meet the product specifications for compact matrix ring blanks in section 5 of the AGR-3/4 DTF Fuel and Capsule Component Material Specifications (SPC-1214, Rev. 0).

After compacting, carbonization, and heat-treatment, all 50 ring blanks were selected for use from the production batch, RDKRS-G001 through G050. As instructed in product inspection plan PIP-20, these 50 ring blanks were randomized and relabeled as RDKRS-Z001 through Z050. A record of the original G-number for each Z-numbered ring blank can be found on inspection report form IRF-20C, in this section. After relabeling, the ring blanks were characterized for product acceptance according to PIP-20. This plan calls for measurement of length, diameter, mass, matrix density, uranium content, and impurity content. Length, diameter, and matrix density were measured on every ring blank. To measure uranium and other specified impurities, ring blanks were burned in air and the ash was leached twice by boiling nitric acid. The acid was analyzed by inductively-coupled plasma mass spectrometry (ICP-MS) to determine the concentration of each impurity. This burn-leach analysis was performed on 12 ring blanks, in four sample groups with 3 ring blanks in each sample group.

After the completion of burn-leach analysis per AGR-CHAR-DAM-44R0, a white ash residue remained in the leaching vessel. The weight of this residue from each sample group (63-66 $\mathrm{mg}$ ) was noted on DRF-44. The observed residue was expected to be mostly $\mathrm{SiO}_{2}$, which is insoluble in nitric acid. Glow discharge mass spectrometry analysis (Section 3) showed that the carbonized RDKrS-0121211 graphite/resin blend had a relatively high silicon content (190 ppm-wt), which mostly came from the natural graphite. Based on this silicon content in the graphite/resin blend, the expected mass of $\mathrm{SiO}_{2}$ residue after burn-off of 3 ring blanks would be $\sim 66 \mathrm{mg}$. This is consistent with the observed weight of the white ash residue remaining after burn-leach of each sample group of ring blanks. For the purpose of the acceptance testing, this ash residue was ignored. Additional analysis of the residue was performed later and is reported in Section 6. It was verified that $\mathrm{Si}$ was the major element in the residue and inclusion of the data for the specified impurities would not affect the results of the acceptance testing.

The following pages show the inspection report forms (IRF-20A, IRF-20B, and IRF-20C) for the RDKRS ring blanks. Following the inspection report forms are the individual data report forms for the measurements that were performed. Inspection report form IRF-20B summarizes the burn-leach results. Inspection report form IRF-20A summarizes all the analyses and provides the statistically calculated acceptance test values for the impurity analyses. These acceptance test values were calculated using a 95\% confidence Student's-t test. Uranium and other impurities were compared against the specification and all values were found to be well below the specified limits. The overall average diameter, length, and matrix density for the ring blank lot are reported on IRF-20A, but the product compliance to these specified parameters was determined on an individual basis for each ring blank (see DRF-43). All ring blanks were within the specified range for mean diameter, length, and matrix density, therefore the ring blank lot met these specification with $100 \%$ confidence. 
Inspection Report Form IRF-20A: AGR-3/4 Matrix Ring Blanks

Procedure: AGR-CHAR-PIP-20 Rev. 1

Ring blank lot ID: RDKRS

Ring blank lot description: Matrix blend with Hexion Durite SC-1008 resin

\begin{tabular}{|c|c|c|c|c|c|c|c|c|c|}
\hline \multirow[b]{2}{*}{ Property } & \multicolumn{4}{|c|}{ Measured Data } & \multirow{2}{*}{$\begin{array}{l}\text { Specification } \\
\text { INL SPC-1214 } \\
\text { Revision 0 }\end{array}$} & \multirow[b]{2}{*}{ Acceptance Criteria } & \multirow{2}{*}{\begin{tabular}{|l|} 
Acceptance \\
Test Value
\end{tabular}} & \multirow{2}{*}{$\begin{array}{l}\text { Pass } \\
\text { or } \\
\text { fail }\end{array}$} & \multirow{2}{*}{$\begin{array}{c}\text { Data } \\
\text { Records }\end{array}$} \\
\hline & $\begin{array}{c}\text { Mean } \\
(x)\end{array}$ & $\begin{array}{l}\text { Std. Dev. } \\
\text { (s) }\end{array}$ & $\begin{array}{l}\text { Measurements } \\
(n)\end{array}$ & $\begin{array}{c}\text { Student's } \\
\text { t value }\end{array}$ & & & & & \\
\hline \multirow{2}{*}{$\begin{array}{l}\text { Ring blank diameter } \\
(\mathbf{m m})\end{array}$} & \multirow{2}{*}{26.01} & \multirow{2}{*}{0.03} & \multirow{2}{*}{50} & & $\begin{array}{c}\text { mean } \\
26.0 \pm 1.0 \\
\end{array}$ & \multicolumn{2}{|l|}{ see DRF-43 } & pass & \multirow{2}{*}{ DRF-43 } \\
\hline & & & & & $\begin{array}{l}\text { dispersion } \\
0 \%<24.5 \\
\end{array}$ & \multicolumn{2}{|l|}{ see DRF-43 } & pass & \\
\hline $\begin{array}{c}\text { Ring blank length } \\
(\mathbf{m m})\end{array}$ & 62.9 & 0.2 & 50 & & $\begin{array}{c}\text { mean } \\
63.0 \pm 2.0 \\
\end{array}$ & \multicolumn{2}{|l|}{ see DRF-43 } & pass & DRF-43 \\
\hline \multirow{2}{*}{$\begin{array}{l}\text { Ring blank matrix density } \\
\qquad(\mathrm{g} / \mathrm{cm} 3)\end{array}$} & \multirow{2}{*}{1.621} & \multirow{2}{*}{0.010} & \multirow{2}{*}{50} & & $\begin{array}{c}\text { mean } \\
1.60 \pm 0.1 \\
\end{array}$ & \multicolumn{2}{|l|}{ see DRF-43 } & pass & \multirow{2}{*}{ DRF-43 } \\
\hline & & & & & $\begin{array}{l}\text { dispersion } \\
0 \%<1.5 \\
\end{array}$ & \multicolumn{2}{|l|}{ see DRF-43 } & pass & \\
\hline $\begin{array}{l}\text { Iron content } \\
\text { (ppmw) }\end{array}$ & 0.06 & 0.03 & 4 & 2.353 & $\begin{array}{l}\text { mean } \\
\leq 20\end{array}$ & $B=x+t s / \sqrt{ } n \leq 20$ & 0.10 & pass & $\begin{array}{l}\text { IRF-20B } \\
\text { DRF-44 }\end{array}$ \\
\hline $\begin{array}{c}\text { Chromium content } \\
\text { (ppmw Fe) }\end{array}$ & 0.0030 & 0.0003 & 4 & 2.353 & $\begin{array}{c}\text { mean } \\
\leq 10 \\
\end{array}$ & $B=x+t s / \sqrt{ } n \leq 10$ & 0.00 & pass & $\begin{array}{l}\text { IRF-20B } \\
\text { DRF-44 } \\
\end{array}$ \\
\hline $\begin{array}{l}\text { Manganese content } \\
(\text { ppmw Mn) }\end{array}$ & 0.0009 & 0.0001 & 4 & 2.353 & $\begin{array}{l}\text { mean } \\
\leq 10 \\
\end{array}$ & $B=x+t s / \sqrt{ } n \leq 10$ & 0.00 & pass & $\begin{array}{l}\text { IRF-20B } \\
\text { DRF-44 } \\
\end{array}$ \\
\hline $\begin{array}{l}\text { Cobalt content } \\
\text { (ppmw Co) }\end{array}$ & 0.0007 & 0.0001 & 4 & 2.353 & $\begin{array}{l}\text { mean } \\
\leq 10 \\
\end{array}$ & $B=x+t s / \sqrt{ } n \leq 10$ & 0.00 & pass & $\begin{array}{l}\text { IRF-20B } \\
\text { DRF-44 }\end{array}$ \\
\hline $\begin{array}{c}\text { Nickel content } \\
\text { (ppmw Ni) }\end{array}$ & 0.0045 & 0.0002 & 4 & 2.353 & $\begin{array}{l}\text { mean } \\
\leq 10 \\
\end{array}$ & $B=x+t s / \sqrt{ } n \leq 10$ & 0.00 & pass & $\begin{array}{l}\text { IRF-20B } \\
\text { DRF-44 } \\
\end{array}$ \\
\hline $\begin{array}{l}\text { Calcium content } \\
\text { (ppmw Ca) }\end{array}$ & 3.7 & 0.3 & 4 & 2.353 & $\begin{array}{l}\text { mean } \\
\leq 45 \\
\end{array}$ & $B=x+t s / \sqrt{ } n \leq 45$ & 4.05 & pass & $\begin{array}{l}\text { IRF-20B } \\
\text { DRF-44 } \\
\end{array}$ \\
\hline $\begin{array}{c}\text { Aluminum content } \\
\text { (ppmw AI) }\end{array}$ & 1.11 & 0.12 & 4 & 2.353 & $\begin{array}{l}\text { mean } \\
\leq 20 \\
\end{array}$ & $B=x+t s / \sqrt{n} \leq 20$ & 1.25 & pass & $\begin{array}{l}\text { IRF-20B } \\
\text { DRF-44 } \\
\end{array}$ \\
\hline $\begin{array}{l}\text { Titanium plus Vanadium content } \\
(\mathrm{ppmw} \text { total } \mathrm{Ti}+\mathrm{V})\end{array}$ & 1.75 & 0.05 & 4 & 2.353 & $\begin{array}{l}\text { mean } \\
\leq 85 \\
\end{array}$ & $B=x+t s / \sqrt{n} \leq 85$ & 1.81 & pass & $\begin{array}{l}\text { IRF-20B } \\
\text { DRF-44 }\end{array}$ \\
\hline $\begin{array}{c}\text { Uranium contamination } \\
(\mathrm{ppmw} U)\end{array}$ & 0.016 & 0.003 & 4 & 2.353 & $\begin{array}{l}\text { mean } \\
\leq 0.5 \\
\end{array}$ & $B=x+t s / \sqrt{ } n \leq 0.5$ & 0.020 & pass & $\begin{array}{l}\text { IRF-20B } \\
\text { DRF-44 }\end{array}$ \\
\hline
\end{tabular}

\section{when $26 m$ m \\ QC Supervisor}

Accept ring blank lot (Yes or No)?

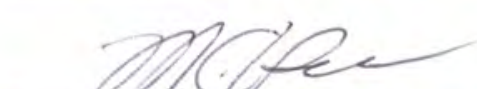

QA Reviewer $5-\mid 6-11$ 


\section{Inspection Report Form IRF-20B: Summary of Impurities in Ring Blanks}

\begin{tabular}{|c|c|}
\hline Procedure: & AGR-CHAR-PIP-20 Rev. 1 \\
\hline Operator: & Fred Montgomery \\
\hline Ring blank lot ID: & RDKRS \\
\hline escription: & ion Durite SC-1008 resin \\
\hline
\end{tabular}

\begin{tabular}{|c|c|c|c|c|c|c|}
\hline Ring blank ID numbers: & $\begin{array}{l}\text { Z026, Z015, } \\
\text { Z014 }\end{array}$ & $\begin{array}{c}\mathrm{z} 003, \mathrm{z} 041, \\
\mathrm{z} 025\end{array}$ & $\begin{array}{c}\text { Z035, Z027, } \\
\text { z040 }\end{array}$ & $\begin{array}{c}\text { Z019, Z036, } \\
\text { z044 }\end{array}$ & Mean & $\begin{array}{l}\text { Standard } \\
\text { Deviation }\end{array}$ \\
\hline \multicolumn{5}{|l|}{ Uranium } & \multicolumn{2}{|c|}{ Uranium } \\
\hline Average impurity content in ring blanks (ppmw) & 0.016 & 0.020 & 0.014 & 0.014 & 0.016 & 0.003 \\
\hline \multicolumn{5}{|l|}{ Iron } & \multicolumn{2}{|c|}{ Iron } \\
\hline Average impurity content in ring blanks (ppmw) & $<0.048$ & $<0.052$ & $<0.041$ & 0.101 & $<0.06$ & 0.03 \\
\hline \multicolumn{5}{|l|}{ Chromium } & \multicolumn{2}{|c|}{ Chromium } \\
\hline Average impurity content in ring blanks (ppmw) & $<0.0031$ & $<0.0034$ & $<0.0026$ & $<0.0029$ & $<0.0030$ & 0.0003 \\
\hline \multicolumn{5}{|l|}{ Manganese } & \multicolumn{2}{|c|}{ Manganese } \\
\hline Average impurity content in ring blanks (ppmw) & $<0.0008$ & $<0.0008$ & $<0.0009$ & $<0.0010$ & $<0.0009$ & 0.0001 \\
\hline \multicolumn{5}{|l|}{ Cobalt } & \multicolumn{2}{|c|}{ Cobalt } \\
\hline Average impurity content in ring blanks (ppmw) & $<0.0007$ & $<0.0007$ & $<0.0007$ & $<0.0008$ & $<0.0007$ & 0.0001 \\
\hline \multicolumn{5}{|l|}{ Nickel } & \multicolumn{2}{|c|}{ Nickel } \\
\hline Average impurity content in ring blanks (ppmw) & $<0.0046$ & $<0.0045$ & $<0.0042$ & $<0.0048$ & $<0.0045$ & 0.0002 \\
\hline \multicolumn{5}{|l|}{ Calcium } & \multicolumn{2}{|c|}{ Calcium } \\
\hline Average impurity content in ring blanks (ppmw) & 3.574 & 3.483 & 3.731 & 4.188 & 3.7 & 0.3 \\
\hline \multicolumn{5}{|l|}{ Aluminum } & \multicolumn{2}{|c|}{ Aluminum } \\
\hline Average impurity content in ring blanks (ppmw) & 1.218 & 1.168 & 0.934 & 1.134 & 1.11 & 0.12 \\
\hline \multicolumn{5}{|l|}{ Titanium } & \multicolumn{2}{|c|}{ Titanium } \\
\hline Average impurity content in ring blanks (ppmw) & 0.567 & 0.551 & 0.576 & 0.614 & 0.58 & 0.03 \\
\hline \multicolumn{5}{|l|}{ Vanadium } & \multicolumn{2}{|c|}{ Vanadium } \\
\hline Average impurity content in ring blanks (ppmw) & 1.149 & 1.164 & 1.152 & 1.215 & 1.17 & 0.03 \\
\hline \multicolumn{5}{|l|}{ Titanium plus Vanadium } & \multicolumn{2}{|c|}{ Titanium plus Vanadium } \\
\hline Average impurity content in ring blanks (ppmw) & 1.715 & 1.716 & 1.728 & 1.829 & 1.75 & 0.05 \\
\hline
\end{tabular}

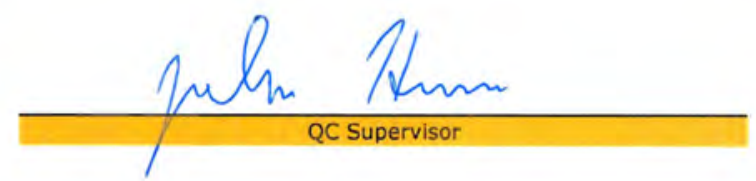

$$
5-16-11
$$




\section{Inspection Report Form IRF-20C: Ring Blank Tracking}

Procedure: AGR-CHAR-PIP-20 Rev. 1

Ring blank lot ID: RDKRS

Ring blank lot description: Matrix blend with Hexion Durite SC-1008 resin

\begin{tabular}{|c|c|}
\hline $\begin{array}{c}\text { Ring Blank } \\
\text { Z Number }\end{array}$ & $\begin{array}{c}\text { Ring Blank } \\
\text { G Number }\end{array}$ \\
\hline \hline Z001 & G016 \\
\hline Z002 & G042 \\
\hline Z003 & G031 \\
\hline Z004 & G036 \\
\hline Z005 & G007 \\
\hline Z006 & G050 \\
\hline Z007 & G017 \\
\hline Z008 & G009 \\
\hline Z009 & G001 \\
\hline Z010 & G039 \\
\hline Z011 & G004 \\
\hline Z012 & G005 \\
\hline Z013 & G021 \\
\hline Z014 & G035 \\
\hline Z015 & G046 \\
\hline
\end{tabular}

\begin{tabular}{|c|c|}
\hline $\begin{array}{c}\text { Ring Blank } \\
\text { Z Number }\end{array}$ & $\begin{array}{c}\text { Ring Blank } \\
\text { G Number }\end{array}$ \\
\hline Z016 & G019 \\
\hline Z017 & G030 \\
\hline Z018 & G026 \\
\hline Z019 & G012 \\
\hline Z020 & G008 \\
\hline Z021 & G032 \\
\hline Z022 & G002 \\
\hline Z023 & G010 \\
\hline Z024 & G011 \\
\hline Z025 & G018 \\
\hline Z026 & G014 \\
\hline Z027 & G049 \\
\hline Z028 & G034 \\
\hline Z029 & G028 \\
\hline Z030 & G022 \\
\hline
\end{tabular}

\begin{tabular}{|c|c|}
\hline $\begin{array}{c}\text { Ring Blank } \\
\text { Z Number }\end{array}$ & $\begin{array}{c}\text { Ring Blank } \\
\text { G Number }\end{array}$ \\
\hline \hline Z031 & G033 \\
\hline Z032 & G044 \\
\hline Z033 & G023 \\
\hline Z034 & G048 \\
\hline Z035 & G038 \\
\hline Z036 & G047 \\
\hline Z037 & G029 \\
\hline Z038 & G040 \\
\hline Z039 & G037 \\
\hline Z040 & G024 \\
\hline Z041 & G045 \\
\hline Z042 & G003 \\
\hline Z043 & G041 \\
\hline Z044 & G025 \\
\hline Z045 & G043 \\
\hline
\end{tabular}

\begin{tabular}{|c|c|}
\hline $\begin{array}{c}\text { Ring Blank } \\
\text { Z Number }\end{array}$ & $\begin{array}{c}\text { Ring Blank } \\
\text { G Number }\end{array}$ \\
\hline Z046 & G013 \\
\hline Z047 & G006 \\
\hline Z048 & G020 \\
\hline Z049 & G027 \\
\hline Z050 & G015 \\
\hline Z051 & \\
\hline Z052 & \\
\hline$Z 053$ & \\
\hline$Z 054$ & \\
\hline$Z 055$ & \\
\hline$Z 056$ & \\
\hline$Z 057$ & \\
\hline$Z 058$ & \\
\hline$Z 059$ & \\
\hline$Z 060$ & \\
\hline
\end{tabular}

Comments

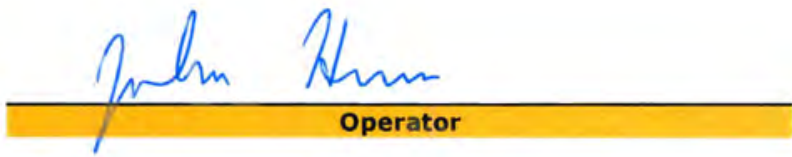

$5-13-11$ 
Data Report Form DRF-43: Matrix Ring Blank Diameter, Length, and Matrix Density

\begin{tabular}{|r|l|}
\hline Procedure: & AGR-CHAR-DAM-43 Rev. 0 \\
\hline Operator: & Dunbar \\
\hline Ring blank lot ID: & RDKRS \\
\hline Ring blank lot description: & Matrix blend with Hexion Durite SC-1008 resin \\
\hline Filename: & IImc-agr\AGRICompactDimensions\RDKRS DRF43R0.xIs \\
\hline
\end{tabular}

\begin{tabular}{|r|l|}
\hline Vertical height gauge calibration due date: & $3 / 22 / 11$ \\
\hline Digital caliper calibration due date: & $5 / 11 / 11$ \\
\hline Gauge blocks calibration due date: & $2 / 27 / 13$ \\
\hline Analytical balance calibration due date: & $11 / 3 / 11$ \\
\hline
\end{tabular}

\begin{tabular}{|r|l|}
\hline Acceptance criteria for ring blank length: & $\geq 61.0$ and $\leq 65.0 \mathrm{~mm}$ \\
\hline Acceptance criteria for ring blank diameter: & $\geq 25.0$ and $\leq 27.0 \mathrm{~mm}$ \\
\hline Acceptance criteria for ring blank matrix density: & $\geq 1.50$ and $\leq 1.70 \mathrm{~g} / \mathrm{cm} 3$ \\
\hline
\end{tabular}

\begin{tabular}{|c|c|c|c|c|c|c|c|c|}
\hline \multirow{2}{*}{$\begin{array}{l}\text { Ring Blank } \\
\text { Z Number }\end{array}$} & \multirow{2}{*}{$\begin{array}{l}\text { Weight } \\
\text { (g) }\end{array}$} & \multirow{2}{*}{$\begin{array}{l}\text { Length } \\
(\mathrm{mm})\end{array}$} & \multicolumn{3}{|c|}{ Diameter $(\mathrm{mm})$} & \multirow{2}{*}{$\begin{array}{l}\text { Volume } \\
(\mathrm{cm} 3)\end{array}$} & \multirow{2}{*}{\begin{tabular}{|c|} 
Matrix Density \\
$(\mathrm{g} / \mathrm{cm} 3)$
\end{tabular}} & \multirow{2}{*}{$\begin{array}{c}\text { Accept? } \\
\text { (pass or fail) }\end{array}$} \\
\hline & & & Middle 1 & Middle 2 & Average & & & \\
\hline Z001 & 54.2801 & 62.746 & 26.00 & 26.02 & 26.01 & 33.339 & 1.63 & pass \\
\hline Z002 & 54.1014 & 62.969 & 26.03 & 26.04 & 26.04 & 33.522 & 1.61 & pass \\
\hline Z003 & 54.2923 & 63.140 & 26.02 & 26.01 & 26.02 & 33.562 & 1.62 & pass \\
\hline Z004 & 54.2285 & 63.125 & 26.01 & 26.01 & 26.01 & 33.541 & 1.62 & pass \\
\hline Z005 & 54.3762 & 62.661 & 26.00 & 26.00 & 26.00 & 33.269 & 1.63 & pass \\
\hline 2006 & 53.9965 & 62.462 & 25.98 & 25.99 & 25.99 & 33.125 & 1.63 & pass \\
\hline Z007 & 54.0788 & 63.007 & 26.00 & 26.00 & 26.00 & 33.452 & 1.62 & pass \\
\hline Z008 & 54.3745 & 62.945 & 26.00 & 26.00 & 26.00 & 33.419 & 1.63 & pass \\
\hline Z009 & 53.9434 & 62.988 & 25.98 & 25.98 & 25.98 & 33.391 & 1.62 & pass \\
\hline$Z 010$ & 53.9241 & 63.157 & 26.03 & 26.04 & 26.04 & 33.622 & 1.60 & pass \\
\hline Z011 & 54.4230 & 63.066 & 25.95 & 25.97 & 25.96 & 33.381 & 1.63 & pass \\
\hline Z012 & 54.3891 & 62.956 & 25.96 & 25.96 & 25.96 & 33.322 & 1.63 & pass \\
\hline Z013 & 53.8372 & 62.769 & 25.99 & 25.97 & 25.98 & 33.275 & 1.62 & pass \\
\hline$Z 014$ & 54.2390 & 62.924 & 26.03 & 26.02 & 26.03 & 33.472 & 1.62 & pass \\
\hline Z015 & 54.2444 & 62.908 & 26.03 & 26.10 & 26.07 & 33.567 & 1.62 & pass \\
\hline Z016 & 53.8742 & 62.927 & 25.99 & 25.99 & 25.99 & 33.384 & 1.61 & pass \\
\hline$Z 017$ & 54.2725 & 62.941 & 26.02 & 26.04 & 26.03 & 33.494 & 1.62 & pass \\
\hline Z018 & 54.0182 & 63.026 & 25.98 & 25.99 & 25.99 & 33.424 & 1.62 & pass \\
\hline Z019 & 54.2684 & 62.462 & 26.00 & 26.00 & 26.00 & 33.163 & 1.64 & pass \\
\hline$Z 020$ & 54.3393 & 63.252 & 25.97 & 25.98 & 25.98 & 33.518 & 1.62 & pass \\
\hline $\mathrm{ZO21}$ & 54.3331 & 62.906 & 26.02 & 26.02 & 26.02 & 33.450 & 1.62 & pass \\
\hline Z022 & 53.9615 & 62.877 & 25.97 & 25.97 & 25.97 & 33.306 & 1.62 & pass \\
\hline Z023 & 54.1593 & 62.857 & 25.98 & 25.98 & 25.98 & 33.321 & 1.63 & pass \\
\hline ZO24 & 54.2871 & 62.717 & 26.00 & 25.99 & 26.00 & 33.285 & 1.63 & pass \\
\hline Z025 & 54.0344 & 62.757 & 26.00 & 26.00 & 26.00 & 33.320 & 1.62 & pass \\
\hline Z026 & 54.2972 & 62.484 & 26.01 & 26.01 & 26.01 & 33.200 & 1.64 & pass \\
\hline$Z 027$ & 54.3196 & 62.972 & 26.05 & 26.05 & 26.05 & 33.562 & 1.62 & pass \\
\hline$Z 028$ & 54.2519 & 62.931 & 26.02 & 26.03 & 26.03 & 33.476 & 1.62 & pass \\
\hline Z029 & 54.2012 & 62.967 & 26.01 & 26.00 & 26.01 & 33.444 & 1.62 & pass \\
\hline Z030 & 53.8570 & 62.967 & 26.00 & 26.00 & 26.00 & 33.431 & 1.61 & pass \\
\hline
\end{tabular}

Comments
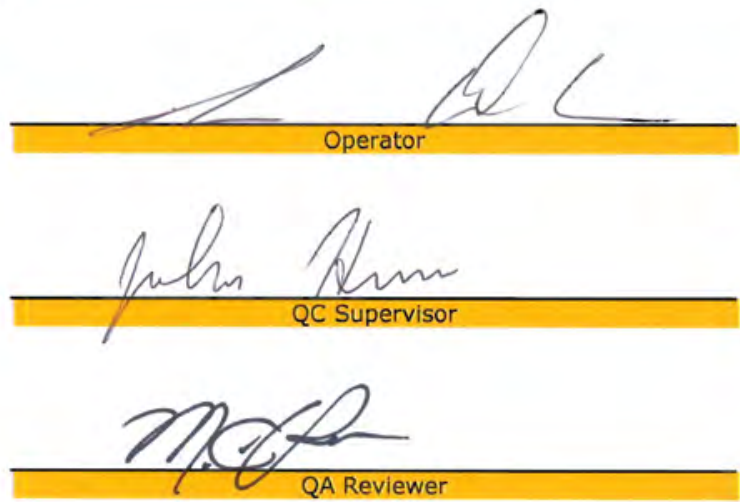

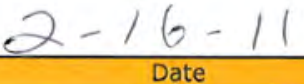

$\frac{2-16-11}{\text { Date }}$

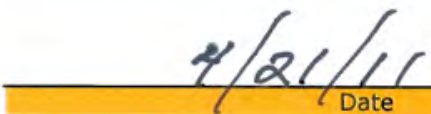


Data Report Form DRF-43: Matrix Ring Blank Diameter, Length, and Matrix Density

\begin{tabular}{|c|c|}
\hline Procedure: & AGR-CHAR-DAM-43 Rev. 0 \\
\hline Operator: & Dunbar \\
\hline Ring blank lot ID: & RDKRS \\
\hline Ring blank lot description: & Matrix blend with Hexion Durite SC-1008 resin \\
\hline Filename: & IImc-agr\AGR\CompactDimensions\RDKRS_DRF43R0.xls \\
\hline Vertical height gauge calibration due date: & $3 / 22 / 11$ \\
\hline Digital caliper calibration due date: & $5 / 11 / 11$ \\
\hline Gauge blocks calibration due date: & $2 / 27 / 13$ \\
\hline Analytical balance calibration due date: & $11 / 3 / 11$ \\
\hline Acceptance criteria for ring blank length: & $\geq 61.0$ and $\leq 65.0 \mathrm{~mm}$ \\
\hline Acceptance criteria for ring blank diameter: & $\geq 25.0$ and $\leq 27.0 \mathrm{~mm}$ \\
\hline tance criteria for ring blank matrix density: & $\geq 1.50$ and $\leq 1.70 \mathrm{~g} / \mathrm{cm} 3$ \\
\hline
\end{tabular}

\begin{tabular}{|c|c|c|c|c|c|c|c|c|}
\hline \multirow{2}{*}{$\begin{array}{l}\text { Ring Blank } \\
\text { Z Number }\end{array}$} & \multirow{2}{*}{$\begin{array}{l}\text { Weight } \\
\text { (g) }\end{array}$} & \multirow{2}{*}{$\begin{array}{l}\text { Length } \\
(\mathrm{mm})\end{array}$} & \multicolumn{3}{|c|}{ Diameter $(\mathrm{mm})$} & \multirow{2}{*}{$\begin{array}{l}\text { Volume } \\
(\mathrm{cm} 3)\end{array}$} & \multirow{2}{*}{\begin{tabular}{|c|} 
Matrix Density \\
$(\mathrm{g} / \mathrm{cm} 3)$
\end{tabular}} & \multirow{2}{*}{$\begin{array}{c}\text { Accept? } \\
\text { (pass or fail) }\end{array}$} \\
\hline & & & Middle 1 & Middle 2 & Average & & & \\
\hline Z031 & 54.2780 & 62.861 & 26.02 & 25.99 & 26.01 & 33.388 & 1.63 & pass \\
\hline Z032 & 53.8416 & 63.079 & 26.03 & 26.02 & 26.03 & 33.555 & 1.60 & pass \\
\hline Z033 & 53.9316 & 63.281 & 26.01 & 26.01 & 26.01 & 33.624 & 1.60 & pass \\
\hline Z034 & 54.1667 & 62.621 & 26.02 & 26.04 & 26.03 & 33.324 & 1.63 & pass \\
\hline$Z 035$ & 54.2288 & 62.923 & 26.03 & 26.05 & 26.04 & 33.511 & 1.62 & pass \\
\hline Z036 & 54.2473 & 62.660 & 25.99 & 26.02 & 26.01 & 33.281 & 1.63 & pass \\
\hline Z037 & 54.1392 & 62.887 & 26.02 & 26.02 & 26.02 & 33.440 & 1.62 & pass \\
\hline$Z 038$ & 54.0811 & 62.927 & 26.03 & 26.03 & 26.03 & 33.487 & 1.61 & pass \\
\hline Z039 & 54.2564 & 62.967 & 25.98 & 26.01 & 26.00 & 33.418 & 1.62 & pass \\
\hline$Z 040$ & 53.9318 & 62.965 & 26.00 & 26.00 & 26.00 & 33.430 & 1.61 & pass \\
\hline Z041 & 54.0419 & 62.782 & 26.01 & 26.01 & 26.01 & 33.358 & 1.62 & pass \\
\hline Z042 & 54.0318 & 62.722 & 25.94 & 25.95 & 25.95 & 33.160 & 1.63 & pass \\
\hline ZO43 & 54.0469 & 63.284 & 26.04 & 26.04 & 26.04 & 33.703 & 1.60 & pass \\
\hline ZO44 & 53.9990 & 63.157 & 26.01 & 26.00 & 26.01 & 33.545 & 1.61 & pass \\
\hline Z045 & 54.3641 & 63.002 & 26.03 & 26.06 & 26.05 & 33.565 & 1.62 & pass \\
\hline Z046 & 54.2171 & 62.411 & 25.97 & 25.97 & 25.97 & 33.059 & 1.64 & pass \\
\hline Z047 & 54.3226 & 62.533 & 25.96 & 25.98 & 25.97 & 33.124 & 1.64 & pass \\
\hline Z048 & 53.8618 & 63.332 & 25.99 & 26.08 & 26.04 & 33.715 & 1.60 & pass \\
\hline Z049 & 53.9414 & 63.009 & 25.99 & 25.99 & 25.99 & 33.428 & 1.61 & pass \\
\hline 2050 & 54.2839 & 62.651 & 26.00 & 26.00 & 26.00 & 33.263 & 1.63 & pass \\
\hline \multicolumn{9}{|l|}{ Z051 } \\
\hline \multicolumn{9}{|l|}{ Z052 } \\
\hline \multicolumn{9}{|l|}{ Z053 } \\
\hline \multirow{2}{*}{\multicolumn{9}{|c|}{$\frac{Z 054}{Z 055}$}} \\
\hline & & & & & & & & \\
\hline \multicolumn{9}{|l|}{ Z056 } \\
\hline \multicolumn{9}{|l|}{ Z057 } \\
\hline \multicolumn{9}{|l|}{ Z058 } \\
\hline$Z 059$ & & & & & & & & \\
\hline Z060 & & & & & & & & \\
\hline
\end{tabular}
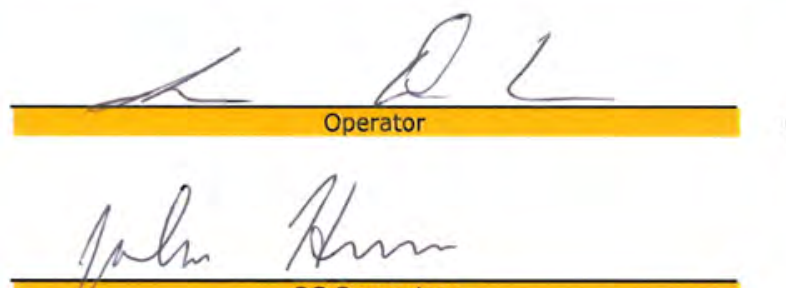

Mope

QA Reviewer

\section{$2-16-11$}

Date

$2-16-11$
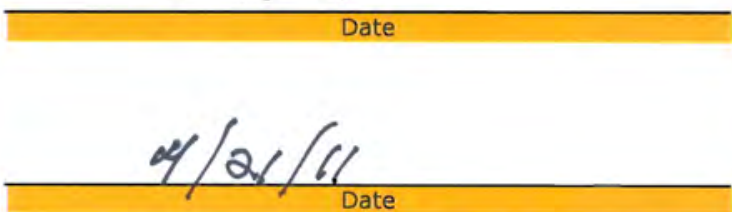
Data Report Form DRF-44: Measurement of Impurities in Matrix Ring Blanks by the Burn-Leach Method

\begin{tabular}{|c|c|}
\hline Procedure: & AGR-CHAR-DAM-44 Rev. 0 \\
\hline Operator: & Fred Montgomery \\
\hline Ring blank lot ID: & RDKRS \\
\hline Ring blank lot description: & Matrix blend with hexion Durite $\mathrm{SC}-1008$ resin \\
\hline Ring blank ID numbers: & $\mathrm{Z} 026, \mathrm{Z} 015, \mathrm{Z} 014$ \\
\hline DRF filename: & IImc-agr|AGRILeac \\
\hline
\end{tabular}

\begin{tabular}{|c|c|c|c|c|}
\hline & & First Leach & Second Leach & Total \\
\hline & Leach solution ID: & BL11030201 & BL11030401 & \\
\hline & Number of ring blanks in sample group: & \multicolumn{2}{|c|}{$\frac{3}{1628}$} & \\
\hline & Total weight of ring blanks in sample group $(\mathrm{g})$ : & \multicolumn{2}{|c|}{162.8} & \\
\hline & Total volume of leach solution $(\mathrm{ml})$ & 36.3 & 35.5 & \\
\hline & Radiochemical laboratory analysis number: & $3580-001$ & $3580-006$ & \\
\hline \multirow{3}{*}{$\mathbf{u}$} & Measured concentration of impurity in solution $(\mu \mathrm{g} / \mathrm{ml})$ : & $6.95 \mathrm{E}-02$ & $2.78 \mathrm{E}-03$ & \multirow[b]{2}{*}{$u$} \\
\hline & Weight of impurity in sample group $(\mu \mathrm{g})$ : & 2.52 & 0.10 & \\
\hline & Average concentration of impurity in ring blanks (ppmw): & 0.0155 & 0.0006 & 0.0161 \\
\hline \multirow{3}{*}{$\mathbf{F e}$} & Measured concentration of impurity in solution $(\mu \mathrm{g} / \mathrm{ml})$ : & $1.74 \mathrm{E}-01$ & $<4.12 \mathrm{E}-02$ & \multirow{2}{*}{$\mathrm{Fe}$} \\
\hline & Weight of impurity in sample group $(\mu \mathrm{g})$ : & 6.32 & $<1.46$ & \\
\hline & Average concentration of impurity in ring blanks (ppmw): & 0.0388 & $<0.0090$ & $<0.0478$ \\
\hline \multirow{3}{*}{$\mathrm{Cr}$} & Measured concentration of impurity in solution $(\mu \mathrm{g} / \mathrm{ml})$ : & $1.18 \mathrm{E}-02$ & $<2.00 \mathrm{E}-03$ & \multirow{2}{*}{$\mathrm{Cr}$} \\
\hline & Weight of impurity in sample group $(\mu \mathrm{g})$ : & 0.43 & $<0.07$ & \\
\hline & Average concentration of impurity in ring blanks (ppmw): & 0.0026 & $<0.0004$ & $<0.0031$ \\
\hline \multirow{3}{*}{ Mn } & Measured concentration of impurity in solution $(\mu \mathrm{g} / \mathrm{ml})$ : & $<1.91 \mathrm{E}-03$ & $<1.91 \mathrm{E}-03$ & \multirow{2}{*}{ Mn } \\
\hline & Weight of impurity in sample group $(\mu \mathrm{g})$ : & $<0.07$ & $<0.07$ & \\
\hline & Average concentration of impurity in ring blanks (ppmw): & $<0.0004$ & $<0.0004$ & $<0.0008$ \\
\hline \multirow{3}{*}{ Co } & Measured concentration of impurity in solution $(\mu \mathrm{g} / \mathrm{ml})$ : & $<1.62 \mathrm{E}-03$ & $<1.62 \mathrm{E}-03$ & \multirow[b]{2}{*}{ Co } \\
\hline & Weight of impurity in sample group $(\mu g)$ : & $<0.06$ & $<0.06$ & \\
\hline & Average concentration of impurity in ring blanks (ppmw): & $<0.0004$ & $<0.0004$ & $<0.0007$ \\
\hline \multirow{3}{*}{$\mathbf{N i}$} & Measured concentration of impurity in solution $(\mu \mathrm{g} / \mathrm{ml})$ : & $1.26 \mathrm{E}-02$ & $<8.00 \mathrm{E}-03$ & \multirow{2}{*}{$\mathrm{Ni}$} \\
\hline & Weight of impurity in sample group $(\mu g)$ : & 0.46 & $<0.28$ & \\
\hline & Average concentration of impurity in ring blanks (ppmw): & 0.0028 & $<0.0017$ & $<0.0046$ \\
\hline \multirow{3}{*}{$\mathbf{C a}$} & Measured concentration of impurity in solution $(\mu \mathrm{g} / \mathrm{ml})$ : & $1.55 E+01$ & $5.37 E-01$ & \multirow{2}{*}{$\mathbf{C a}$} \\
\hline & Weight of impurity in sample group $(\mu \mathrm{g})$ : & 562.65 & 19.06 & \\
\hline & Average concentration of impurity in ring blanks (ppmw): & 3.4565 & 0.1171 & 3.5736 \\
\hline \multirow{3}{*}{ Al } & Measured concentration of impurity in solution $(\mu \mathrm{g} / \mathrm{ml})$ : & $5.20 E+00$ & $2.66 \mathrm{E}-01$ & \multirow{2}{*}{ Al } \\
\hline & Weight of impurity in sample group $(\mu \mathrm{g})$ : & 188.76 & 9.44 & \\
\hline & Average concentration of impurity in ring blanks (ppmw): & 1.1596 & 0.0580 & 1.2176 \\
\hline \multirow{3}{*}{$\mathbf{T i}$} & Measured concentration of impurity in solution $(\mu \mathrm{g} / \mathrm{ml})$ : & $2.51 \mathrm{E}+00$ & $3.28 \mathrm{E}-02$ & \multirow{2}{*}{$\mathrm{Ti}$} \\
\hline & Weight of impurity in sample group $(\mu \mathrm{g})$ : & 91.11 & 1.16 & \\
\hline & Average concentration of impurity in ring blanks (ppmw): & 0.5597 & 0.0072 & 0.5669 \\
\hline \multirow{3}{*}{$\mathbf{v}$} & Measured concentration of impurity in solution $(\mu \mathrm{g} / \mathrm{ml})$ : & $5.14 E+00$ & $1.06 \mathrm{E}-02$ & \multirow[b]{2}{*}{$\mathbf{v}$} \\
\hline & Weight of impurity in sample group $(\mu \mathrm{g})$ : & 186.58 & 0.38 & \\
\hline & Average concentration of impurity in ring blanks (ppmw): & 1.1462 & 0.0023 & 1.1485 \\
\hline
\end{tabular}

Weight of ash after nitric acid leaching was $0.0661 \mathrm{~g}$

\section{Comments}

Data checked against the official results of analyses for RMAL3580 by FCM on 4/21/2011.

\section{Troel C. Montgomeuy}

$$
4-21-2011
$$


Data Report Form DRF-44: Measurement of Impurities in Matrix Ring Blanks by the Burn-Leach Method

\begin{tabular}{|c|c|}
\hline Procedure: & AGR-CHAR-DAM-44 Rev. 0 \\
\hline Operator: & Fred Montgomery \\
\hline Ring blank lot ID: & RDKRS \\
\hline Ring blank lot description: & Matrix blend with hexion Durite SC- 1008 resin \\
\hline Ring blank ID numbers: & Z003, Z041, Z025 \\
\hline DRF filename: & \Imc-agr\AGR\LeachBurnLeach\RDKRS DRF44R0.xls \\
\hline
\end{tabular}

\begin{tabular}{|c|c|c|c|c|}
\hline & & First Leach & Second Leach & Total \\
\hline & Leach solution ID: & BL11030202 & BL11030402 & \\
\hline & Number of ring blanks in sample group: & \multicolumn{2}{|l|}{ 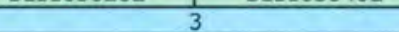 } & \\
\hline & Total weight of ring blanks in sample group & \multicolumn{2}{|c|}{162.4} & \\
\hline & Total volume of leach solution $(\mathrm{ml})$ : & 34.5 & 31.3 & \\
\hline & Radiochemical laboratory analysis number: & $3580-002$ & $3580-007$ & \\
\hline \multirow{3}{*}{$\mathbf{u}$} & Measured concentration of impurity in solution $(\mu \mathrm{g} / \mathrm{ml})$ : & $8.71 \mathrm{E}-02$ & $6.63 E-03$ & \multirow[b]{2}{*}{$\mathbf{u}$} \\
\hline & Weight of impurity in sample group $(\mu \mathrm{g}):$ & 3.00 & 0.21 & \\
\hline & Average concentration of impurity in ring blanks (ppmw): & 0.0185 & 0.0013 & 0.0198 \\
\hline \multirow{3}{*}{$\mathrm{Fe}$} & Measured concentration of impurity in solution $(\mu \mathrm{g} / \mathrm{ml})$ : & $2.08 \mathrm{E}-01$ & $<4.12 \mathrm{E}-02$ & \multirow{2}{*}{$\mathrm{Fe}$} \\
\hline & Weight of impurity in sample group $(\mu \mathrm{g})$ : & 7.18 & $<1.29$ & \\
\hline & Average concentration of impurity in ring blanks (ppmw): & 0.0442 & $<0.0079$ & $<0.0521$ \\
\hline \multirow{3}{*}{$\mathrm{Cr}$} & Measured concentration of impurity in solution $(\mu \mathrm{g} / \mathrm{ml})$ : & $1.41 \mathrm{E}-02$ & $<2.00 \mathrm{E}-03$ & \multirow[b]{2}{*}{$\mathrm{Cr}$} \\
\hline & Weight of impurity in sample group $(\mu \mathrm{g})$ : & 0.49 & $<0.06$ & \\
\hline & Average concentration of impurity in ring blanks (ppmw): & 0.0030 & $<0.0004$ & $<0.0034$ \\
\hline \multirow{3}{*}{ Mn } & Measured concentration of impurity in solution $(\mu \mathrm{g} / \mathrm{ml})$ : & $<1.91 \mathrm{E}-03$ & $<1.91 \mathrm{E}-03$ & \multirow{2}{*}{ Mn } \\
\hline & Weight of impurity in sample group $(\mu \mathrm{g})$ : & $<0.07$ & $<0.06$ & \\
\hline & Average concentration of impurity in ring blanks (ppmw): & $<0.0004$ & $<0.0004$ & $<0.0008$ \\
\hline \multirow{3}{*}{ Co } & Measured concentration of impurity in solution $(\mu \mathrm{g} / \mathrm{ml})$ : & $<1.62 \mathrm{E}-03$ & $<1.62 \mathrm{E}-03$ & \multirow[b]{2}{*}{ Co } \\
\hline & Weight of impurity in sample group $(\mu \mathrm{g}):$ & $<0.06$ & $<0.05$ & \\
\hline & Average concentration of impurity in ring blanks (ppmw): & $<0.0003$ & $<0.0003$ & $<0.0007$ \\
\hline \multirow{3}{*}{$\mathbf{N i}$} & Measured concentration of impurity in solution $(\mu \mathrm{g} / \mathrm{ml})$ : & $1.37 E-02$ & $<8.00 \mathrm{E}-03$ & \multirow{2}{*}{ Ni } \\
\hline & Weight of impurity in sample group $(\mu \mathrm{g}):$ & 0.47 & $<0.25$ & \\
\hline & Average concentration of impurity in ring blanks (ppmw): & 0.0029 & $<0.0015$ & $<0.0045$ \\
\hline \multirow{3}{*}{$\mathrm{Ca}$} & Measured concentration of impurity in solution $(\mu \mathrm{g} / \mathrm{ml})$ : & $1.59 \mathrm{E}+01$ & $5.42 \mathrm{E}-01$ & \multirow[b]{2}{*}{$\mathrm{Ca}$} \\
\hline & Weight of impurity in sample group $(\mu \mathrm{g})$ : & 548.55 & 16.96 & \\
\hline & Average concentration of impurity in ring blanks (ppmw): & 3.3784 & 0.1045 & 3.4829 \\
\hline \multirow{3}{*}{ Al } & Measured concentration of impurity in solution $(\mu \mathrm{g} / \mathrm{ml})$ : & $5.29 E+00$ & $2.28 \mathrm{E}-01$ & \multirow{2}{*}{ Al } \\
\hline & Weight of impurity in sample group $(\mu \mathrm{g}):$ & 182.51 & 7.14 & \\
\hline & Average concentration of impurity in ring blanks (ppmw): & 1.1240 & 0.0440 & 1.1680 \\
\hline \multirow{3}{*}{$\mathbf{T i}$} & Measured concentration of impurity in solution $(\mu \mathrm{g} / \mathrm{ml})$ : & $2.55 E+00$ & 5.01E-02 & \multirow{2}{*}{$\mathrm{Ti}$} \\
\hline & Weight of impurity in sample group $(\mu \mathrm{g}):$ & 87.98 & 1.57 & \\
\hline & Average concentration of impurity in ring blanks (ppmw): & 0.5418 & 0.0097 & 0.5515 \\
\hline \multirow{3}{*}{$\mathbf{v}$} & Measured concentration of impurity in solution $(\mu \mathrm{g} / \mathrm{ml})$ : & $5.46 E+00$ & $2.15 E-02$ & \multirow{2}{*}{ v } \\
\hline & Weight of impurity in sample group $(\mu \mathrm{g})$ : & 188.37 & 0.67 & \\
\hline & Average concentration of impurity in ring blanks (ppmw): & 1.1601 & 0.0041 & 1.1643 \\
\hline
\end{tabular}

Weight of ash after nitric acid leaching was $0.0632 \mathrm{~g}$,

Comments

Data checked against the official results of analyses for RMAL3580 by FCM on 4/21/2011.

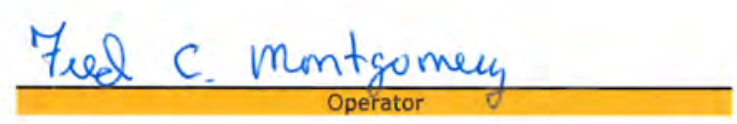

$4-21-2011$ 
Data Report Form DRF-44: Measurement of Impurities in Matrix Ring Blanks by the Burn-Leach Method

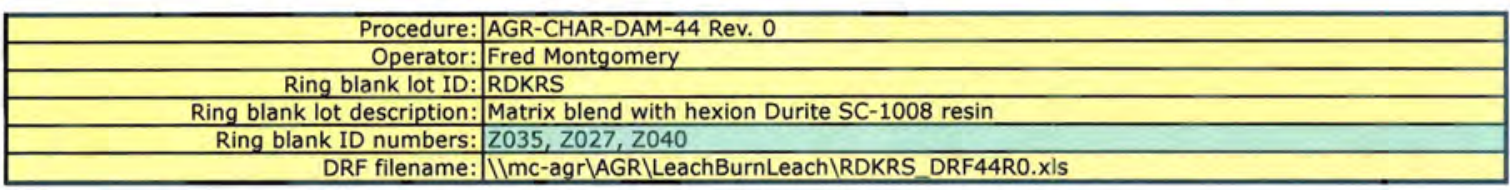

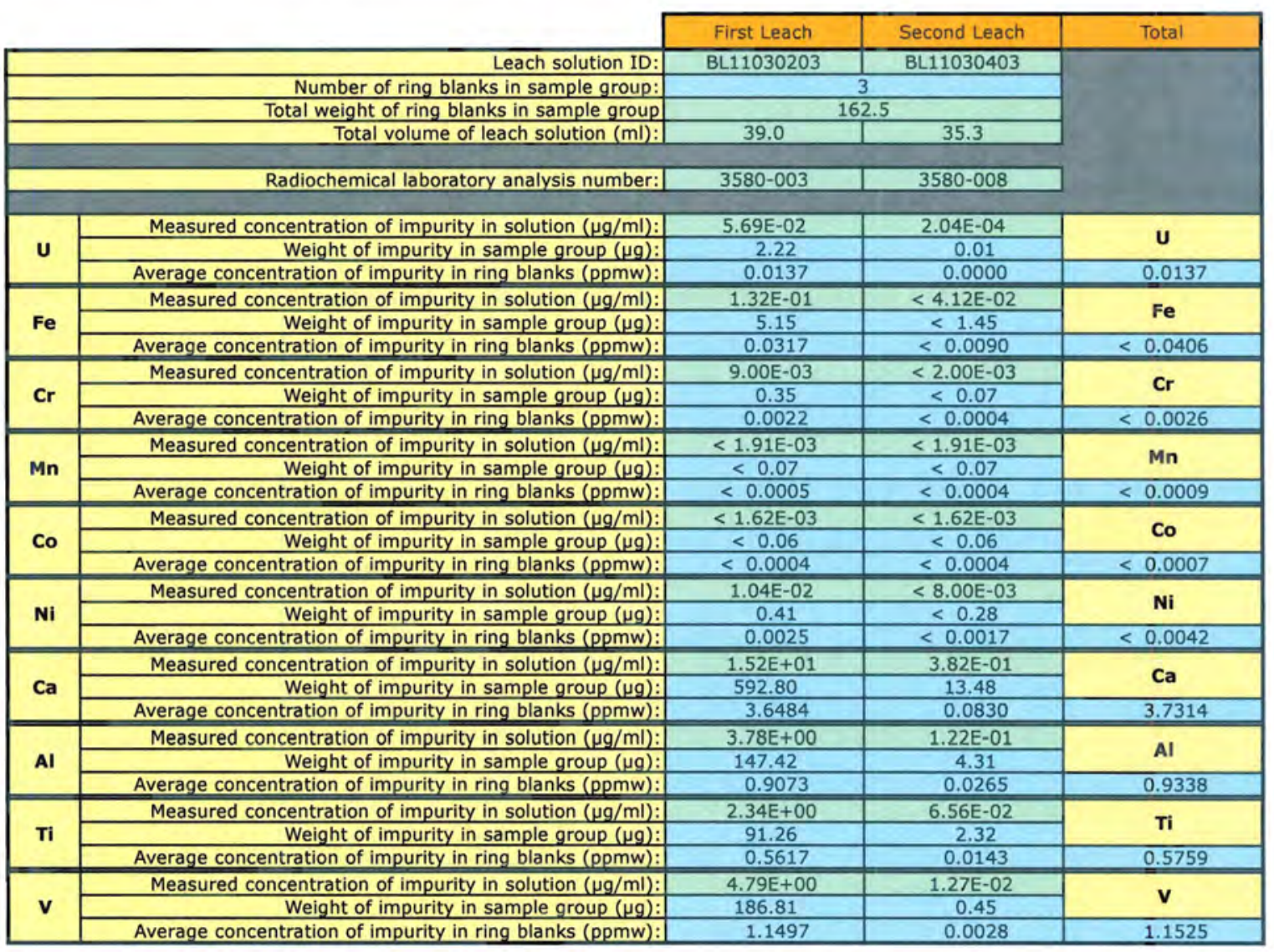

Weight of ash after nitric acid leaching was $0.0656 \mathrm{~g}$.

Comments

Data checked against the official results of analyses for RMAL3580 by FCM on 4/21/2011.

Fued c. montromely
$4-21-2011$

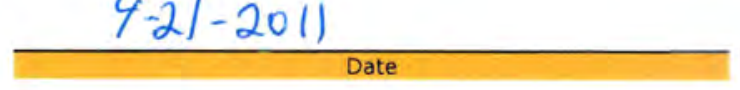


Data Report Form DRF-44: Measurement of Impurities in Matrix Ring Blanks by the Burn-Leach Method

\begin{tabular}{|c|c|}
\hline Procedure: & AGR-CHAR-DAM-44 Rev. 0 \\
\hline Operator: & Fred Montgomery \\
\hline Ring blank lot ID: & RDKRS \\
\hline Ring blank lot description: & Matrix blend with hexion Durite $\mathrm{SC}-1008$ resin \\
\hline Ring blank ID numbers: & $\mathrm{Z019}, \mathrm{Z036}, \mathrm{ZO44}$ \\
\hline DRF filename: & IImc-agr\AGR\LeachBurnLeach \RDKRS_DRF44R0.xls \\
\hline
\end{tabular}

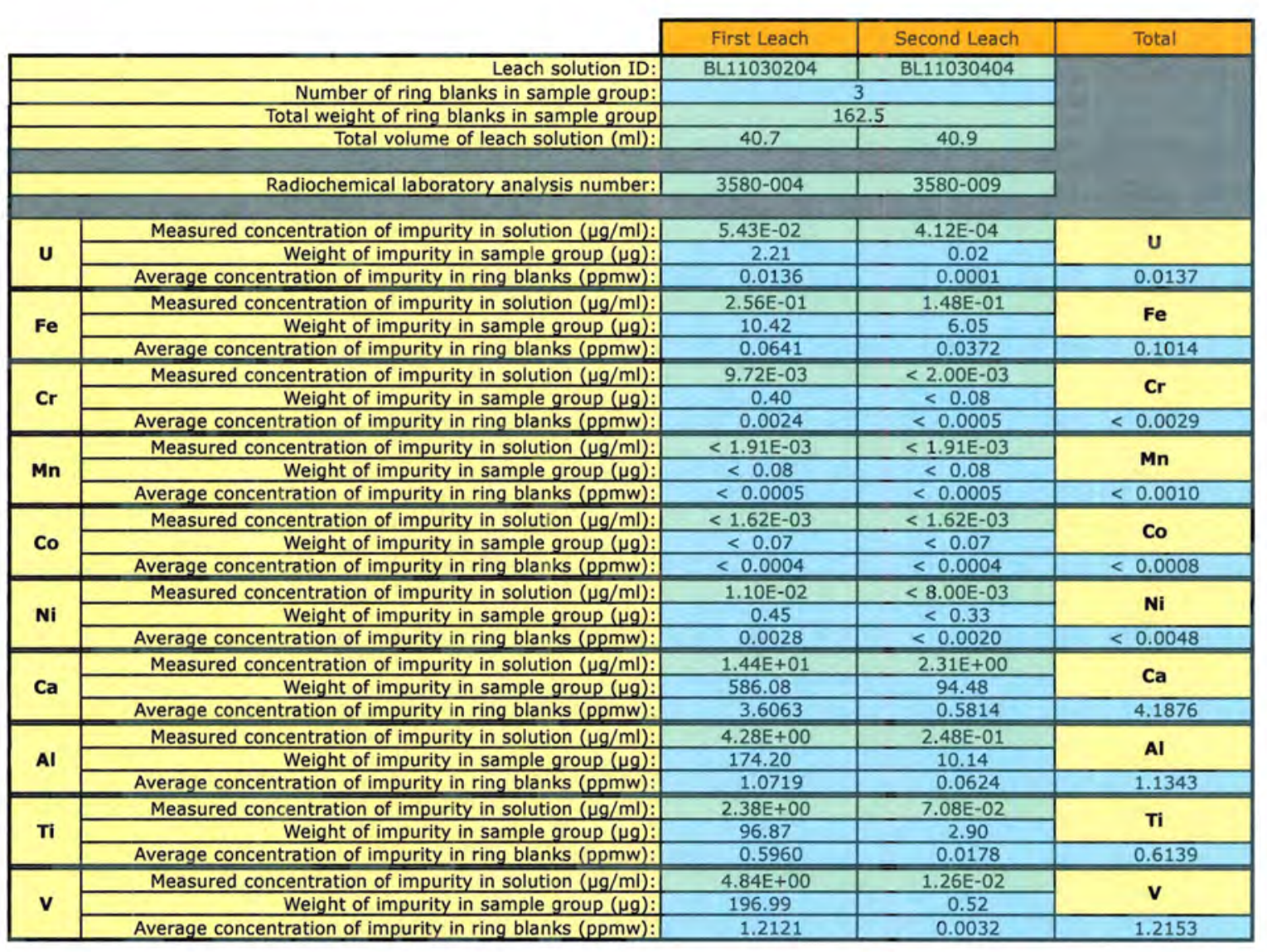

Weight of ash after nitric acid leaching was $0.0630 \mathrm{~g}$.

\section{Fued c. Muntyoneun}

$$
4-21-2011
$$




\section{Data Report Form DRF-44: Measurement of Impurities in Matrix Ring Blanks by the Burn-Leach Method}

\begin{tabular}{|c|c|}
\hline \multirow{2}{*}{\multicolumn{2}{|c|}{$\begin{aligned} & \text { Procedure } \text { AGR-CHAR-DAM-44 Rev. } 0 \\
& \text { Operator: } \text { Fred Montgomery } \\
&\end{aligned}$}} \\
\hline & \\
\hline Ring blank lot ID: & RDKRS \\
\hline Ring blank lot description: & Matrix blend with hexion Durite SC- 1008 resin \\
\hline Ring blank ID numbers: & Blank \\
\hline DRF filename: & IImc-agr\AGR\LeachBurnLeach\RDKRS_DRF44R0.xIs \\
\hline
\end{tabular}

\begin{tabular}{|c|c|c|c|c|}
\hline & 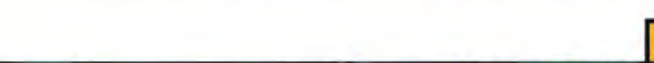 & First Leach & Second Leach & Total \\
\hline & Leach solution ID: & BL11030205 & BL11030405 & \\
\hline & Number of ring blanks in sample group: & & & \\
\hline & Total weight of ring blanks in sample group & & & \\
\hline & Total volume of leach solution $(\mathrm{ml})$ : & 34.7 & 32.7 & \\
\hline & Radiochemical laboratory analysis number: & $3580-005$ & $3580-010$ & \\
\hline \multirow{3}{*}{ u } & Measured concentration of impurity in solution $(\mu \mathrm{g} / \mathrm{ml})$ : & $6.76 \mathrm{E}-03$ & $9.72 \mathrm{E}-04$ & \multirow{3}{*}{ u } \\
\hline & Weight of impurity in sample group $(\mu \mathrm{g})$ : & 0.23 & 0.03 & \\
\hline & Average concentration of impurity in ring blanks (ppmw): & & & \\
\hline \multirow{3}{*}{$\mathbf{F e}$} & Measured concentration of impurity in solution $(\mu \mathrm{g} / \mathrm{ml})$ : & $1.95 \mathrm{E}-01$ & $<4.12 \mathrm{E}-02$ & \multirow{3}{*}{$\mathbf{F e}$} \\
\hline & Weight of impurity in sample group $(\mu g)$ : & 6.77 & $<1.35$ & \\
\hline & Average concentration of impurity in ring blanks (ppmw): & & & \\
\hline \multirow{3}{*}{ Cr } & Measured concentration of impurity in solution $(\mu \mathrm{g} / \mathrm{ml})$ : & $1.17 \mathrm{E}-02$ & $<2.00 \mathrm{E}-03$ & \multirow{3}{*}{ Cr } \\
\hline & Weight of impurity in sample group $(\mu \mathrm{g})$ : & 0.41 & $<0.07$ & \\
\hline & Average concentration of impurity in ring blanks (ppmw): & & & \\
\hline \multirow{3}{*}{ Mn } & Measured concentration of impurity in solution $(\mu \mathrm{g} / \mathrm{ml})$ : & $<1.91 \mathrm{E}-03$ & $<1.91 \mathrm{E}-03$ & \multirow{3}{*}{ Mn } \\
\hline & Weight of impurity in sample group $(\mu \mathrm{g})$ : & $<0.07$ & $<0.06$ & \\
\hline & Average concentration of impurity in ring blanks (ppmw): & & & \\
\hline \multirow{3}{*}{ Co } & Measured concentration of impurity in solution $(\mu \mathrm{g} / \mathrm{ml})$ : & $<1.62 \mathrm{E}-03$ & $<1.62 \mathrm{E}-03$ & \multirow{3}{*}{ Co } \\
\hline & Weight of impurity in sample group $(\mu g)$ : & $<0.06$ & $<0.05$ & \\
\hline & Average concentration of impurity in ring blanks (ppmw): & & & \\
\hline \multirow{3}{*}{$\mathbf{N i}$} & Measured concentration of impurity in solution $(\mu \mathrm{g} / \mathrm{ml})$ : & $8.40 \mathrm{E}-03$ & $<8.00 \mathrm{E}-03$ & \multirow{3}{*}{ Ni } \\
\hline & Weight of impurity in sample group $(\mu \mathrm{g})$ : & 0.29 & $<0.26$ & \\
\hline & Average concentration of impurity in ring blanks (ppmw): & & & \\
\hline \multirow{3}{*}{$\mathbf{C a}$} & Measured concentration of impurity in solution $(\mu \mathrm{g} / \mathrm{ml})$ : & $2.48 \mathrm{E}-01$ & $1.81 \mathrm{E}-01$ & \multirow{3}{*}{ Ca } \\
\hline & Weight of impurity in sample group $(\mu \mathrm{g})$ : & 8.61 & 5.92 & \\
\hline & Average concentration of impurity in ring blanks (ppmw): & & & \\
\hline \multirow{3}{*}{ Al } & Measured concentration of impurity in solution $(\mu \mathrm{g} / \mathrm{ml})$ : & $1.78 \mathrm{E}-01$ & $7.28 \mathrm{E}-02$ & \multirow{3}{*}{ Al } \\
\hline & Weight of impurity in sample group $(\mu \mathrm{g})$ : & 6.18 & 2.38 & \\
\hline & Average concentration of impurity in ring blanks (ppmw): & & & \\
\hline \multirow{3}{*}{$\mathbf{T i}$} & Measured concentration of impurity in solution $(\mu \mathrm{g} / \mathrm{ml})$ : & $4.62 \mathrm{E}-02$ & $9.60 \mathrm{E}-03$ & \multirow{3}{*}{$\mathbf{T i}$} \\
\hline & Weight of impurity in sample group $(\mu g)$ : & 1.60 & 0.31 & \\
\hline & Average concentration of impurity in ring blanks (ppmw): & & & \\
\hline \multirow{3}{*}{$\mathbf{v}$} & Measured concentration of impurity in solution $(\mu \mathrm{g} / \mathrm{ml})$ : & $<2.00 \mathrm{E}-03$ & $<2.00 \mathrm{E}-03$ & \multirow{3}{*}{$\mathbf{v}$} \\
\hline & Weight of impurity in sample group $(\mu g)$ : & $<0.07$ & $<0.07$ & \\
\hline & Average concentration of impurity in ring blanks (ppmw): & & & \\
\hline
\end{tabular}




\section{Additional Characterization of RDKRS Ring Blanks for Information Only}

Additional analysis of the burn-leach solutions was performed to measure $\mathrm{Ba}$ and lanthanide impurities. The following pages include a modified version of IRF-20B, which summarizes the results, and the individual modified data report forms (DRF-44) for each sample group. A significant amount (40 ppm-wt) of barium was detected. 


\section{Inspection Report Form IRF-20B: Summary of Impurities in Ring Blanks}

\begin{tabular}{|c|c|}
\hline Procedure: & AGR-CHAR-PIP-20 Rev. 1 \\
\hline Operator: & Fred Montgomery \\
\hline Ring blank lot ID: & RDKRS \\
\hline Ring blank lot description: & Matrix blend with hexion Durite SC-1008 resin \\
\hline
\end{tabular}

\begin{tabular}{|c|c|c|c|c|c|c|}
\hline Ring blank ID numbers: & $\begin{array}{c}\mathrm{Z} 026, \mathrm{Z} 015 \\
\mathrm{z} 014\end{array}$ & $\begin{array}{c}\mathrm{Z} 003, \mathrm{Z041} \\
\mathrm{Z} 025\end{array}$ & $\begin{array}{c}\text { Z035, Z027 } \\
\text { z040 }\end{array}$ & $\begin{array}{c}\text { Z019, z036, } \\
\text { z044 }\end{array}$ & Mean & $\begin{array}{l}\text { Standard } \\
\text { Deviation }\end{array}$ \\
\hline Barium & & & & & \multicolumn{2}{|c|}{ Barium } \\
\hline Average impurity content in ring blanks (ppmw) & 49.6 & 41.0 & 32.0 & 36.3 & 39.7 & 7.6 \\
\hline Lanthanum & & & & & \multicolumn{2}{|c|}{ Lanthanum } \\
\hline Average impurity content in ring blanks (ppmw) & 0.586 & 0.581 & 0.581 & 0.599 & 0.587 & 0.009 \\
\hline Cerium & & & & & \multicolumn{2}{|c|}{ Cerium } \\
\hline \begin{tabular}{|l|} 
Average impurity content in ring blanks (ppmw) \\
\end{tabular} & 0.205 & 0.204 & 0.203 & 0.209 & 0.205 & 0.003 \\
\hline Neodymium & & & & & \multicolumn{2}{|c|}{ Neodymium } \\
\hline Europium & & & & & \multicolumn{2}{|c|}{ Europium } \\
\hline Average impurity content in ring blanks (ppmw) & 0.0198 & 0.0191 & 0.0198 & 0.0192 & 0.0195 & 0.0004 \\
\hline Gadolinium & & & & & \multicolumn{2}{|c|}{ Gadolinium } \\
\hline Average impurity content in ring blanks (ppmw) & 0.056 & 0.049 & 0.042 & 0.049 & 0.049 & 0.006 \\
\hline Dysprosium & & & & & \multicolumn{2}{|c|}{ Dysprosium } \\
\hline Average impurity content in ring blanks (ppmw) & $<0.0097$ & $<0.0096$ & $<0.0099$ & $<0.0100$ & $<0.0098$ & 0.0002 \\
\hline Erbium & & & & & \multicolumn{2}{|c|}{ Erbium } \\
\hline Average impurity content in ring blanks (ppmw) & $<0.0075$ & $<0.0074$ & $<0.0076$ & $<0.0077$ & $<0.0075$ & 0.0001 \\
\hline Average impurity content in ring blanks (ppmw) & $<0.0013$ & $<0.0013$ & $<0.0014$ & $<0.0014$ & $<0.00136$ & 0.00003 \\
\hline
\end{tabular}

\section{Comments}

Additional analysis of $\mathrm{Ba}$ and Lanthanides was performed for information only.

After burning and leaching with nitric acid, a white ash remained in the leach vessel. This ash was probably mostly silica and will be analyzed separately.

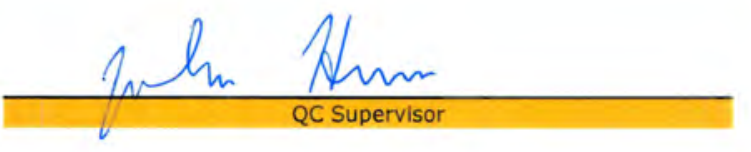

$$
5-16-11
$$




\begin{tabular}{|c|c|}
\hline Procedure: & AGR-CHAR-DAM-44 Rev. 0 \\
\hline Operator: & Fred Montgomery \\
\hline Ring blank lot ID: & RDKRS \\
\hline Ring blank lot description: & Matrix blend with hexion Durite SC-1008 resin \\
\hline Ring blank ID numbers: & $\mathrm{Z} 026, \mathrm{Z} 015, \mathrm{Z} 014$ \\
\hline DRF filename: & 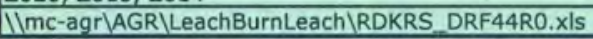 \\
\hline
\end{tabular}

\begin{tabular}{|c|c|c|c|c|}
\hline & & First Leach & Second Leach & Total \\
\hline & Leach solution ID: & BL11030201 & BL11030401 & \\
\hline & Number of ring blanks in sample group: & \multicolumn{2}{|l|}{ 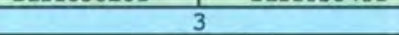 } & \\
\hline & Total weight of ring blanks in sample group $(\mathrm{g})$ : & \multicolumn{2}{|c|}{162.8} & \\
\hline & Total volume of leach solution $(\mathrm{ml}):$ & 36.3 & 35.5 & \\
\hline & Radiochemical laboratory analysis number: & $3580-001$ & $3580-006$ & \\
\hline \multirow{3}{*}{$\mathbf{B a}$} & Measured concentration of impurity in solution $(\mu \mathrm{g} / \mathrm{ml})$ : & $1.13 E+02$ & $1.12 \mathrm{E}+02$ & \multirow[b]{2}{*}{ Ba } \\
\hline & Weight of impurity in sample group $(\mu \mathrm{g})$ : & 4101.90 & 3976.00 & \\
\hline & Average concentration of impurity in ring blanks (ppmw): & 25.1987 & 24.4253 & 49.6240 \\
\hline \multirow{3}{*}{ La } & Measured concentration of impurity in solution $(\mu \mathrm{g} / \mathrm{ml})$ : & $2.62 E+00$ & $9.84 \mathrm{E}-03$ & \multirow{2}{*}{ La } \\
\hline & Weight of impurity in sample group $(\mu \mathrm{g})$ : & 95.11 & 0.35 & \\
\hline & Average concentration of impurity in ring blanks (ppmw): & 0.5843 & 0.0021 & 0.5864 \\
\hline \multirow{3}{*}{$\mathrm{Ce}$} & Measured concentration of impurity in solution $(\mu \mathrm{g} / \mathrm{ml})$ : & $9.16 \mathrm{E}-01$ & $2.60 \mathrm{E}-03$ & \multirow{2}{*}{$\mathrm{Ce}$} \\
\hline & Weight of impurity in sample group $(\mu \mathrm{g}):$ & 33.25 & 0.09 & \\
\hline & Average concentration of impurity in ring blanks (ppmw): & 0.2043 & 0.0006 & 0.2048 \\
\hline \multirow{3}{*}{ Nd } & Measured concentration of impurity in solution $(\mu \mathrm{g} / \mathrm{ml})$ : & $4.76 \mathrm{E}-01$ & $9.20 \mathrm{E}-04$ & \multirow{2}{*}{ Nd } \\
\hline & Weight of impurity in sample group $(\mu \mathrm{g})$ : & 17.28 & 0.03 & \\
\hline & Average concentration of impurity in ring blanks (ppmw): & 0.1061 & 0.0002 & 0.1063 \\
\hline \multirow{3}{*}{ Eu } & Measured concentration of impurity in solution $(\mu \mathrm{g} / \mathrm{ml})$ : & $8.76 E-02$ & $1.28 \mathrm{E}-03$ & \multirow{2}{*}{ Eu } \\
\hline & Weight of impurity in sample group $(\mu \mathrm{g})$ : & 3.18 & 0.05 & \\
\hline & Average concentration of impurity in ring blanks (ppmw): & 0.0195 & 0.0003 & 0.0198 \\
\hline \multirow{3}{*}{ Gd } & Measured concentration of impurity in solution $(\mu \mathrm{g} / \mathrm{ml})$ : & $1.23 \mathrm{E}-01$ & $1.32 E-01$ & \multirow{2}{*}{ Gd } \\
\hline & Weight of impurity in sample group $(\mu \mathrm{g})$ : & 4.46 & 4.69 & \\
\hline & Average concentration of impurity in ring blanks (ppmw): & 0.0274 & 0.0288 & 0.0562 \\
\hline \multirow{3}{*}{ Dy } & Measured concentration of impurity in solution $(\mu \mathrm{g} / \mathrm{ml})$ : & 4.35E-02 & $<2.00 \mathrm{E}-04$ & \multirow{2}{*}{ Dy } \\
\hline & Weight of impurity in sample group $(\mu \mathrm{g})$ : & 1.58 & $<0.01$ & \\
\hline & Average concentration of impurity in ring blanks (ppmw): & 0.0097 & $<0.0000$ & $<0.0097$ \\
\hline \multirow{3}{*}{ Er } & Measured concentration of impurity in solution $(\mu \mathrm{g} / \mathrm{ml})$ : & $3.34 \mathrm{E}-02$ & $<2.00 \mathrm{E}-04$ & \multirow{2}{*}{ Er } \\
\hline & Weight of impurity in sample group $(\mu g)$ : & 1.21 & $<0.01$ & \\
\hline & Average concentration of impurity in ring blanks (ppmw): & 0.0074 & $<0.0000$ & $<0.0075$ \\
\hline \multirow{3}{*}{ Lu } & Measured concentration of impurity in solution $(\mu \mathrm{g} / \mathrm{ml})$ : & $5.84 E-03$ & $<2.00 \mathrm{E}-04$ & \multirow{2}{*}{ Lu } \\
\hline & Weight of impurity in sample group $(\mu \mathrm{g})$ : & 0.21 & $<0.01$ & \\
\hline & Average concentration of impurity in ring blanks (ppmw): & 0.0013 & $<0.0000$ & $<0.0013$ \\
\hline
\end{tabular}

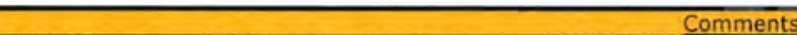

Additional analysis of Ba and Lanthanides was performed for information only.

Weight of ash after nitric acid leaching was $0.0661 \mathrm{~g}$.

Data checked by FCM against the official results of analyses for RMAL3580 on 4/21/2011.

Feed C. Montsomey

$$
4-21-2011
$$


Data Report Form DRF-44: Measurement of Impurities in Matrix Rina Blanks by the Burn-Leach Method

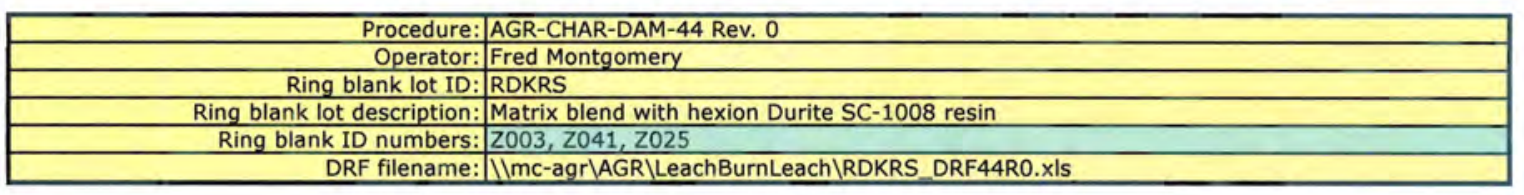

\begin{tabular}{|c|c|c|c|c|}
\hline & & First Leach & Second Leach & Total \\
\hline & Leach solution ID: & BL11030202 & BL.11030402 & \\
\hline & Number of ring blanks in sample group: & \multicolumn{2}{|r|}{ 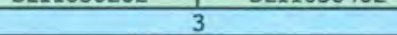 } & \\
\hline & Total weight of ring blanks in sample group & \multicolumn{2}{|c|}{162.4} & \\
\hline & Total volume of leach solution $(\mathrm{ml})$ : & 34.5 & 31.3 & \\
\hline & Radiochemical laboratory analysis number: & $3580-002$ & $3580-007$ & \\
\hline \multirow{3}{*}{$\mathbf{B a}$} & Measured concentration of impurity in solution $(\mu \mathrm{g} / \mathrm{ml})$ : & $1.12 E+02$ & $8.94 E+01$ & \multirow{2}{*}{$\mathbf{B a}$} \\
\hline & Weight of impurity in sample group $(\mu g)$ : & 3864.00 & 2798.22 & \\
\hline & Average concentration of impurity in ring blanks (ppmw): & 23.7976 & 17.2337 & 41.0312 \\
\hline \multirow{3}{*}{ La } & Measured concentration of impurity in solution $(\mu \mathrm{g} / \mathrm{ml})$ : & $2.72 E+00$ & $1.57 \mathrm{E}-02$ & \multirow[b]{2}{*}{ La } \\
\hline & Weight of impurity in sample group $(\mu \mathrm{g})$ : & 93.84 & 0.49 & \\
\hline & Average concentration of impurity in ring blanks (ppmw): & 0.5779 & 0.0030 & 0.5810 \\
\hline \multirow{3}{*}{ Ce } & Measured concentration of impurity in solution $(\mu \mathrm{g} / \mathrm{ml})$ : & $9.56 \mathrm{E}-01$ & $4.24 \mathrm{E}-03$ & \multirow{2}{*}{$\mathrm{Ce}$} \\
\hline & Weight of impurity in sample group $(\mu \mathrm{g})$ : & 32.98 & 0.13 & \\
\hline & Average concentration of impurity in ring blanks (ppmw): & 0.2031 & 0.0008 & 0.2039 \\
\hline \multirow{3}{*}{ Nd } & Measured concentration of impurity in solution $(\mu \mathrm{g} / \mathrm{ml})$ : & $4.84 E-01$ & $1.60 \mathrm{E}-03$ & \multirow{2}{*}{ Nd } \\
\hline & Weight of impurity in sample group $(\mu g)$ : & 16.70 & 0.05 & \\
\hline & Average concentration of impurity in ring blanks (ppmw): & 0.1028 & 0.0003 & 0.1031 \\
\hline \multirow{3}{*}{ Eu } & Measured concentration of impurity in solution $(\mu \mathrm{g} / \mathrm{ml})$ : & $8.90 \mathrm{E}-02$ & $1.24 \mathrm{E}-03$ & \multirow{2}{*}{ Eu } \\
\hline & Weight of impurity in sample group $(\mu \mathrm{g})$ : & 3.07 & 0.04 & \\
\hline & Average concentration of impurity in ring blanks (ppmw): & 0.0189 & 0.0002 & 0.0191 \\
\hline \multirow{3}{*}{ Gd } & Measured concentration of impurity in solution $(\mu \mathrm{g} / \mathrm{ml})$ : & $1.27 \mathrm{E}-01$ & $1.16 \mathrm{E}-01$ & \multirow[b]{2}{*}{ Gd } \\
\hline & Weight of impurity in sample group $(\mu \mathrm{g})$ : & 4.38 & 3.63 & \\
\hline & Average concentration of impurity in ring blanks (ppmw): & 0.0270 & 0.0224 & 0.0493 \\
\hline \multirow{3}{*}{ Dy } & Measured concentration of impurity in solution $(\mu \mathrm{g} / \mathrm{ml})$ : & $4.52 E-02$ & $<2.00 \mathrm{E}-04$ & \multirow{2}{*}{ Dy } \\
\hline & Weight of impurity in sample group $(\mu \mathrm{g})$ : & 1.56 & $<0.01$ & \\
\hline & Average concentration of impurity in ring blanks (ppmw): & 0.0096 & $<0.0000$ & $<0.0096$ \\
\hline \multirow{3}{*}{ Er } & Measured concentration of impurity in solution $(\mu \mathrm{g} / \mathrm{ml})$ : & $3.45 \mathrm{E}-02$ & $<2.00 \mathrm{E}-04$ & \multirow{2}{*}{ Er } \\
\hline & Weight of impurity in sample group $(\mu \mathrm{g})$ : & 1.19 & $<0.01$ & \\
\hline & Average concentration of impurity in ring blanks (ppmw): & 0.0073 & $<0.0000$ & $<0.0074$ \\
\hline \multirow{3}{*}{ Lu } & Measured concentration of impurity in solution $(\mu \mathrm{g} / \mathrm{ml})$ : & $6.08 \mathrm{E}-03$ & $<2.00 \mathrm{E}-04$ & \multirow{2}{*}{ Lu } \\
\hline & Weight of impurity in sample group $(\mu \mathrm{g})$ : & 0.21 & $<0.01$ & \\
\hline & Average concentration of impurity in ring blanks (ppmw): & 0.0013 & $<0.0000$ & $<0.0013$ \\
\hline
\end{tabular}

\section{Fied c. montgomen}

$4-21-2011$ 
Data Report Form DRF-44: Measurement of Impurities in Matrix Ring Blanks by the Burn-Leach Method

\begin{tabular}{|c|c|}
\hline \multirow{2}{*}{$\begin{array}{l}\text { Procedure } \\
\text { Operator }\end{array}$} & AGR-CHAR-DAM-44 Rev. 0 \\
\hline & Fred Montgomery \\
\hline Ring blank lot ID: & RDKRS \\
\hline Ring blank lot description: & Matrix blend with hexion Durite SC- 1008 resin \\
\hline Ring blank ID numbers: & $2035, Z 027, Z 040$ \\
\hline DRF filename: & 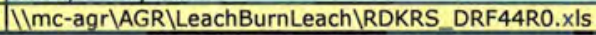 \\
\hline
\end{tabular}

\begin{tabular}{|c|c|c|c|c|}
\hline & & First Leach & Second Leach & Total \\
\hline & Leach solution ID: & BL11030203 & BL11030403 & \\
\hline & Number of ring blanks in sample group: & & \\
\hline & Total weight of ring blanks in sample group & \multicolumn{2}{|c|}{162.5} & \\
\hline & Total volume of leach solution $(\mathrm{ml})$ : & 39.0 & 35.3 & \\
\hline & Radiochemical laboratory analysis number: & $3580-003$ & $3580-008$ & \\
\hline \multirow{3}{*}{ Ba } & Measured concentration of impurity in solution $(\mu \mathrm{g} / \mathrm{ml})$ : & $7.74 E+01$ & $6.18 \mathrm{E}+01$ & \multirow{2}{*}{ Ba } \\
\hline & Weight of impurity in sample group $(\mu \mathrm{g})$ : & 3018.60 & 2181.54 & \\
\hline & Average concentration of impurity in ring blanks (ppmw): & 18.5782 & 13.4264 & 32.0046 \\
\hline \multirow{3}{*}{ La } & Measured concentration of impurity in solution $(\mu \mathrm{g} / \mathrm{ml})$ : & $2.41 \mathrm{E}+00$ & $1.23 \mathrm{E}-02$ & \multirow[b]{2}{*}{ La } \\
\hline & Weight of impurity in sample group $(\mu \mathrm{g})$ : & 93.99 & 0.43 & \\
\hline & Average concentration of impurity in ring blanks (ppmw): & 0.5785 & 0.0027 & 0.5811 \\
\hline \multirow{3}{*}{ Ce } & Measured concentration of impurity in solution $(\mu \mathrm{g} / \mathrm{ml})$ : & $8.44 \mathrm{E}-01$ & $2.64 \mathrm{E}-03$ & \multirow{2}{*}{$\mathrm{Ce}$} \\
\hline & Weight of impurity in sample group $(\mu \mathrm{g})$ : & 32.92 & 0.09 & \\
\hline & Average concentration of impurity in ring blanks (ppmw): & 0.2026 & 0.0006 & 0.2032 \\
\hline \multirow{3}{*}{ Nd } & Measured concentration of impurity in solution $(\mu \mathrm{g} / \mathrm{ml})$ : & $4.32 \mathrm{E}-01$ & $8.40 \mathrm{E}-04$ & \multirow{2}{*}{ Nd } \\
\hline & Weight of impurity in sample group $(\mu g)$ : & 16.85 & 0.03 & \\
\hline & Average concentration of impurity in ring blanks (ppmw): & 0.1037 & 0.0002 & 0.1039 \\
\hline \multirow{3}{*}{ Eu } & Measured concentration of impurity in solution $(\mu \mathrm{g} / \mathrm{ml})$ : & $8.19 \mathrm{E}-02$ & $8.00 \mathrm{E}-04$ & \multirow{2}{*}{ Eu } \\
\hline & Weight of impurity in sample group $(\mu g)$ : & 3.19 & 0.03 & \\
\hline & Average concentration of impurity in ring blanks (ppmw): & 0.0197 & 0.0002 & 0.0198 \\
\hline \multirow{3}{*}{ Gd } & Measured concentration of impurity in solution $(\mu \mathrm{g} / \mathrm{ml})$ : & $1.04 \mathrm{E}-01$ & $8.03 E-02$ & \multirow{2}{*}{ Gd } \\
\hline & Weight of impurity in sample group $(\mu \mathrm{g})$ : & 4.06 & 2.83 & \\
\hline & Average concentration of impurity in ring blanks (ppmw): & 0.0250 & 0.0174 & 0.0424 \\
\hline \multirow{3}{*}{ Dy } & Measured concentration of impurity in solution $(\mu \mathrm{g} / \mathrm{ml})$ : & $4.12 \mathrm{E}-02$ & $<2.00 \mathrm{E}-04$ & \multirow{2}{*}{ Dy } \\
\hline & Weight of impurity in sample group $(\mu \mathrm{g})$ : & 1.61 & $<0.01$ & \\
\hline & Average concentration of impurity in ring blanks (ppmw): & 0.0099 & $<0.0000$ & $<0.0099$ \\
\hline \multirow{3}{*}{ Er } & Measured concentration of impurity in solution $(\mu \mathrm{g} / \mathrm{ml})$ : & $3.15 \mathrm{E}-02$ & $<2.00 \mathrm{E}-04$ & \multirow{2}{*}{ Er } \\
\hline & Weight of impurity in sample group $(\mu \mathrm{g})$ : & 1.23 & $<0.01$ & \\
\hline & Average concentration of impurity in ring blanks (ppmw): & 0.0076 & $<0.0000$ & $<0.0076$ \\
\hline \multirow{3}{*}{ Lu } & Measured concentration of impurity in solution $(\mu \mathrm{g} / \mathrm{ml})$ : & $5.56 \mathrm{E}-03$ & $<2.00 \mathrm{E}-04$ & \multirow{2}{*}{ Lu } \\
\hline & Weight of impurity in sample group $(\mu \mathrm{g})$ : & 0.22 & $<0.01$ & \\
\hline & Average concentration of impurity in ring blanks (ppmw): & 0.0013 & $<0.0000$ & $<0.0014$ \\
\hline
\end{tabular}

Additional analysis of Ba and Lanthanides was performed for information only.

Weight of ash after nitric acid leaching was $0.0656 \mathrm{~g}$.

Data checked by FCM against the official results of analyses for RMAL3580 on 4/21/2011.

\section{Foedc. Mentromem Operator}

$4-21-2011$

Date 
Data Report Form DRF-44: Measurement of Impurities in Matrix Ring Blanks by the Burn-Leach Method

\begin{tabular}{|r|l|}
\hline Procedure: & AGR-CHAR-DAM-44 Rev. 0 \\
\hline Operator: & Fred Montgomery \\
\hline Ring blank lot ID: & RDKRS \\
\hline Ring blank lot description: & Matrix blend with hexion Durite SC-1008 resin \\
\hline Ring blank ID numbers: & Z019, Z036, Z044 \\
\hline DRF filename: & $\backslash$ Imc-agr\AGR\LeachBurnLeach \RDKRS DRF44R0.xIs \\
\hline
\end{tabular}

\begin{tabular}{|c|c|c|c|c|}
\hline & & First Leach & Second Leach & Total \\
\hline & Leach solution ID: & BL11030204 & BL11030404 & \\
\hline & Number of ring blanks in sample group: & \multicolumn{2}{|c|}{3} & \\
\hline & Total weight of ring blanks in sample group & \multicolumn{2}{|c|}{162.5} & \\
\hline & Total volume of leach solution $(\mathrm{ml})$ : & 40.7 & 40.9 & \\
\hline & Radiochemical laboratory analysis number: & $3580-004$ & $3580-009$ & \\
\hline \multirow{3}{*}{ Ba } & Measured concentration of impurity in solution $(\mu \mathrm{g} / \mathrm{ml})$ : & $6.74 \mathrm{E}+01$ & $7.71 E+01$ & \multirow[b]{2}{*}{$\mathbf{B a}$} \\
\hline & Weight of impurity in sample group $(\mu \mathrm{g})$ : & 2743.18 & 3153.39 & \\
\hline & Average concentration of impurity in ring blanks (ppmw): & 16.8794 & 19.4035 & 36.2830 \\
\hline \multirow{3}{*}{ La } & Measured concentration of impurity in solution $(\mu \mathrm{g} / \mathrm{ml})$ : & $2.38 \mathrm{E}+00$ & $1.26 \mathrm{E}-02$ & \multirow{2}{*}{ La } \\
\hline & Weight of impurity in sample group $(\mu \mathrm{g}):$ & 96.87 & 0.52 & \\
\hline & Average concentration of impurity in ring blanks (ppmw): & 0.5960 & 0.0032 & 0.5992 \\
\hline \multirow{3}{*}{$\mathrm{Ce}$} & Measured concentration of impurity in solution $(\mu \mathrm{g} / \mathrm{ml})$ : & $8.32 \mathrm{E}-01$ & $3.08 \mathrm{E}-03$ & \multirow{2}{*}{$\mathrm{Ce}$} \\
\hline & Weight of impurity in sample group $(\mu \mathrm{g}):$ & 33.86 & 0.13 & \\
\hline & Average concentration of impurity in ring blanks (ppmw): & 0.2084 & 0.0008 & 0.2091 \\
\hline \multirow{3}{*}{ Nd } & Measured concentration of impurity in solution $(\mu \mathrm{g} / \mathrm{ml})$ : & $4.32 \mathrm{E}-01$ & $1.04 \mathrm{E}-03$ & \multirow{2}{*}{ Nd } \\
\hline & Weight of impurity in sample group $(\mu \mathrm{g}):$ & 17.58 & 0.04 & \\
\hline & Average concentration of impurity in ring blanks (ppmw): & 0.1082 & 0.0003 & 0.1085 \\
\hline \multirow{3}{*}{ Eu } & Measured concentration of impurity in solution $(\mu \mathrm{g} / \mathrm{ml})$ : & 7.57E-02 & $9.60 \mathrm{E}-04$ & \multirow{2}{*}{ Eu } \\
\hline & Weight of impurity in sample group $(\mu g)$ : & 3.08 & 0.04 & \\
\hline & Average concentration of impurity in ring blanks (ppmw): & 0.0190 & 0.0002 & 0.0192 \\
\hline \multirow{3}{*}{ Gd } & Measured concentration of impurity in solution $(\mu \mathrm{g} / \mathrm{ml})$ : & $9.52 E-02$ & 9.87E-02 & \multirow{2}{*}{ Gd } \\
\hline & Weight of impurity in sample group $(\mu \mathrm{g})$ : & 3.87 & 4.04 & \\
\hline & Average concentration of impurity in ring blanks (ppmw): & 0.0238 & 0.0248 & 0.0487 \\
\hline \multirow{3}{*}{ Dy } & Measured concentration of impurity in solution $(\mu \mathrm{g} / \mathrm{ml})$ : & $3.97 E-02$ & $<2.00 \mathrm{E}-04$ & \multirow{2}{*}{ Dy } \\
\hline & Weight of impurity in sample group $(\mu \mathrm{g})$ : & 1.62 & $<0.01$ & \\
\hline & Average concentration of impurity in ring blanks (ppmw): & 0.0099 & $<0.0001$ & $<0.0100$ \\
\hline \multirow{3}{*}{ Er } & Measured concentration of impurity in solution $(\mu \mathrm{g} / \mathrm{ml})$ : & $3.04 \mathrm{E}-02$ & $<2.00 \mathrm{E}-04$ & \multirow{2}{*}{ Er } \\
\hline & Weight of impurity in sample group $(\mu \mathrm{g}):$ & 1.24 & $<0.01$ & \\
\hline & Average concentration of impurity in ring blanks ( $\mathrm{ppmw}$ ): & 0.0076 & $<0.0001$ & $<0.0077$ \\
\hline \multirow{3}{*}{ Lu } & Measured concentration of impurity in solution $(\mu \mathrm{g} / \mathrm{ml})$ : & $5.36 \mathrm{E}-03$ & $<2.00 E-04$ & \multirow{2}{*}{ Lu } \\
\hline & Weight of impurity in sample group $(\mu \mathrm{g})$ : & 0.22 & $<0.01$ & \\
\hline & Average concentration of impurity in ring blanks (ppmw): & 0.0013 & $<0.0001$ & $<0.0014$ \\
\hline
\end{tabular}

\section{Comments}

Additional analysis of Ba and Lanthanides was performed for information only.

Weight of ash after nitric acid leaching was $0.0630 \mathrm{~g}$.

Data checked by FCM against the official results of analyses for RMAL 3580 on 4/21/2011.

Feed c. mindonmey
$4-21-2011$

Date 
Data Report Form DRF-44: Measurement of Impurities in Matrix Ring Blanks by the Burn-Leach Method

\begin{tabular}{|c|c|}
\hline Procedure: & AGR-CHAR-DAM-44 Rev. 0 \\
\hline Operator: & Fred Montgomery \\
\hline Ring blank lot ID: & RDKRS \\
\hline Ring blank lot description: & Matrix blend with hexion Durite SC- 1008 resin \\
\hline Ring blank ID numbers: & Blank \\
\hline DRF filename: & 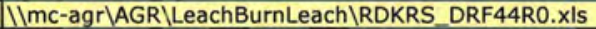 \\
\hline
\end{tabular}

\begin{tabular}{|c|c|c|c|c|}
\hline & & First Leach & Second Leach & Total \\
\hline & Leach solution ID: & BL11030205 & BL11030405 & \\
\hline & Number of ring blanks in sample group: & \multicolumn{2}{|l|}{ 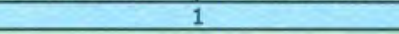 } & \\
\hline \multicolumn{2}{|r|}{ Total weight of ring blanks in sample group } & & & \\
\hline \multicolumn{2}{|r|}{ Total volume of leach solution $(\mathrm{ml})$ : } & 34.7 & 32.7 & \\
\hline \multicolumn{2}{|r|}{ Radiochemical laboratory analysis number: } & $3580-005$ & $3580-010$ & \\
\hline \multirow{3}{*}{ Ba } & Measured concentration of impurity in solution $(\mu \mathrm{g} / \mathrm{ml})$ : & $<2.00 \mathrm{E}-02$ & $<2.00 \mathrm{E}-02$ & \multirow{3}{*}{ Ba } \\
\hline & Weight of impurity in sample group $(\mu \mathrm{g})$ : & $<0.69$ & $<0.65$ & \\
\hline & Average concentration of impurity in ring blanks (ppmw): & & & \\
\hline \multirow{3}{*}{ La } & Measured concentration of impurity in solution $(\mu \mathrm{g} / \mathrm{ml})$ : & $<2.00 \mathrm{E}-04$ & $2.40 \mathrm{E}-04$ & \multirow{3}{*}{ La } \\
\hline & Weight of impurity in sample group $(\mu \mathrm{g})$ : & $<0.01$ & 0.01 & \\
\hline & Average concentration of impurity in ring blanks (ppmw): & & & \\
\hline \multirow{3}{*}{$\mathrm{Ce}$} & Measured concentration of impurity in solution $(\mu \mathrm{g} / \mathrm{ml})$ : & $<2.00 \mathrm{E}-04$ & $<2.00 \mathrm{E}-04$ & \multirow{3}{*}{$\mathrm{Ce}$} \\
\hline & Weight of impurity in sample group $(\mu \mathrm{g})$ : & $<0.01$ & $<0.01$ & \\
\hline & Average concentration of impurity in ring blanks (ppmw): & & & \\
\hline \multirow{3}{*}{ Nd } & Measured concentration of impurity in solution $(\mu \mathrm{g} / \mathrm{ml})$ : & $<2.00 \mathrm{E}-04$ & $<2.00 \mathrm{E}-04$ & \multirow{3}{*}{ Nd } \\
\hline & Weight of impurity in sample group $(\mu \mathrm{g})$ : & $<0.01$ & $<0.01$ & \\
\hline & Average concentration of impurity in ring blanks (ppmw): & & & \\
\hline \multirow{3}{*}{ Eu } & Measured concentration of impurity in solution $(\mu \mathrm{g} / \mathrm{ml})$ : & $<2.00 \mathrm{E}-04$ & $<2.00 \mathrm{E}-04$ & \multirow{3}{*}{ Eu } \\
\hline & Weight of impurity in sample group $(\mu \mathrm{g})$ : & $<0.01$ & $<0.01$ & \\
\hline & Average concentration of impurity in ring blanks (ppmw): & & & \\
\hline \multirow{3}{*}{ Gd } & Measured concentration of impurity in solution $(\mu \mathrm{g} / \mathrm{ml})$ : & $<2.00 \mathrm{E}-04$ & $<2.00 \mathrm{E}-04$ & \multirow{3}{*}{ Gd } \\
\hline & Weight of impurity in sample group $(\mu \mathrm{g})$ : & $<0.01$ & $<0.01$ & \\
\hline & Average concentration of impurity in ring blanks (ppmw): & & & \\
\hline \multirow{3}{*}{ Dy } & Measured concentration of impurity in solution $(\mu \mathrm{g} / \mathrm{ml})$ : & $<2.00 \mathrm{E}-04$ & $<2.00 \mathrm{E}-04$ & \multirow{3}{*}{ Dy } \\
\hline & Weight of impurity in sample group $(\mu \mathrm{g})$ : & $<0.01$ & $<0.01$ & \\
\hline & Average concentration of impurity in ring blanks (ppmw): & & & \\
\hline \multirow{3}{*}{ Er } & Measured concentration of impurity in solution $(\mathrm{\mu g} / \mathrm{ml})$ : & $<2.00 \mathrm{E}-04$ & $<2.00 \mathrm{E}-04$ & \multirow{3}{*}{ Er } \\
\hline & Weight of impurity in sample group $(\mu \mathrm{g})$ : & $<0.01$ & $<0.01$ & \\
\hline & Average concentration of impurity in ring blanks (ppmw): & & & \\
\hline \multirow{3}{*}{ Lu } & Measured concentration of impurity in solution $(\mu \mathrm{g} / \mathrm{ml})$ : & $<2.00 \mathrm{E}-04$ & $<2.00 E-04$ & \multirow{3}{*}{ Lu } \\
\hline & Weight of impurity in sample group $(\mu \mathrm{g})$ : & $<0.01$ & $<0.01$ & \\
\hline & Average concentration of impurity in ring blanks (ppmw): & & & \\
\hline
\end{tabular}

\section{Foed C. Mntomeney}

$$
4-21-2011
$$




\section{Analysis of Ash Residue Remaining After Burn-Leach}

As reported in Section 4, a white ash residue remained in the leaching vessel after the completion of burn-leach analysis. This ash residue was analyzed by ICP-MS after microwave digestion and the results are reported on the following pages. IRF-20B Supplemental summarizes the analysis for the list of specified impurities and adds the data from the microwave digestion analysis of the ash residue to that obtained from the standard burn-leach analysis using boiling nitric acid. Following the inspection report form are the individual data report forms for the measurements that were performed. The impurity content detected in the ash residue was essentially insignificant (typically around $10 \%$ of what was detected by the burn-leach analysis), with the exception of $\mathrm{Ti}$ (where the total impurity content increased by $\sim 50 \%$ ). However, the magnitude of the Ti impurity content is still very low compared to what is allowed by the specification.

As expected, the dominant metallic impurity in the ash residue was silicon $(96-98 \mathrm{wt} \%$ of the impurities detected). Based on the amount of $\mathrm{Si}$ detected in the ash residue, the average $\mathrm{Si}$ impurity content in the ring blanks was around 100 ppmw. A measureable amount of Ba was also detected in the ash residue (1-4 ppmw), which was $\leq 10 \%$ of what was detected by the burnleach analysis reported in Section 5. 
Inspection Report Form IRF-20B Supplemental: Summary of Impurities in Ring Blanks Including Analysis of Ash Residue

\begin{tabular}{|r|l|}
\hline Procedure: & AGR-CHAR-PIP-20 Rev. 1 \\
\hline Operator: & Fred Montgomery \\
\hline Ring blank lot ID: & RDKRS \\
\hline Ring blank lot description: & Matrix blend with hexion Durite SC-1008 resin \\
\hline
\end{tabular}

\begin{tabular}{|c|c|c|c|c|c|c|}
\hline Ring blank ID numbers: & $\begin{array}{c}\text { Z026, zo15, } \\
\text { z014 }\end{array}$ & $\begin{array}{c}\mathrm{z} 2003, \mathrm{z} 2041 \\
\mathrm{z} 025\end{array}$ & $\begin{array}{c}\text { Z035, zo27, } \\
\text { z040 }\end{array}$ & $\begin{array}{c}\text { Z019, Z036, } \\
\text { Z044 }\end{array}$ & Mean & $\begin{array}{l}\text { Standard } \\
\text { Deviation }\end{array}$ \\
\hline \multicolumn{5}{|l|}{ Uranium } & \multicolumn{2}{|c|}{ Uranium } \\
\hline Impurity content detected by acid leach (ppmw) & 0.016 & 0.020 & 0.014 & 0.014 & 0.016 & 0.003 \\
\hline Impurity content detected in ash (ppmw) & 0.0009 & 0.0014 & 0.0015 & 0.0008 & 0.0011 & 0.0003 \\
\hline Total impurity content in ring blanks (ppmw) & 0.017 & 0.021 & 0.015 & 0.015 & 0.017 & 0.003 \\
\hline \multicolumn{5}{|l|}{ Iron } & \multicolumn{2}{|c|}{ Iron } \\
\hline Impurity content detected by acid leach (ppmw) & $<0.048$ & $<0.052$ & $<0.041$ & 0.101 & $<0.06$ & 0.03 \\
\hline Impurity content detected in ash (ppmw) & 0.001 & 0.001 & 0.012 & 0.023 & 0.009 & 0.010 \\
\hline Total impurity content in ring blanks (ppmw) & $<0.049$ & $<0.053$ & $<0.053$ & 0.124 & $<0.07$ & 0.04 \\
\hline \multicolumn{5}{|l|}{ Chromium } & \multicolumn{2}{|c|}{ Chromium } \\
\hline Impurity content detected by acid leach (ppmw) & $<0.0031$ & $<0.0034$ & $<0.0026$ & $<0.0029$ & $<0.0030$ & 0.0003 \\
\hline Impurity content detected in ash (ppmw) & $<0.0004$ & $<0.0005$ & $<0.0005$ & $<0.0005$ & $<0.0005$ & 0.0000 \\
\hline Total impurity content in ring blanks (ppmw) & $<0.0035$ & $<0.0039$ & $<0.0031$ & $<0.0034$ & $<0.0035$ & 0.0003 \\
\hline \multicolumn{5}{|l|}{ Manganese } & \multicolumn{2}{|c|}{ Manganese } \\
\hline Impurity content detected by acid leach (ppmw) & $<0.0008$ & $<0.0008$ & $<0.0009$ & $<0.0010$ & $<0.0009$ & 0.0001 \\
\hline Impurity content detected in ash (ppmw) & $<0.0004$ & $<0.0005$ & $<0.0005$ & $<0.0005$ & $<0.0005$ & 0.0000 \\
\hline Total impurity content in ring blanks (ppmw) & $<0.0013$ & $<0.0013$ & $<0.0014$ & $<0.0015$ & $<0.0013$ & 0.0001 \\
\hline \multicolumn{5}{|l|}{ Cobalt } & \multicolumn{2}{|c|}{ Cobalt } \\
\hline Impurity content detected by acid leach (ppmw) & $<0.0007$ & $<0.0007$ & $<0.0007$ & $<0.0008$ & $<0.0007$ & 0.0001 \\
\hline Impurity content detected in ash (ppmw) & 0.0002 & $<0.0002$ & $<0.0002$ & $<0.0002$ & $<0.0002$ & 0.0000 \\
\hline Total impurity content in ring blanks (ppmw) & $<0.0009$ & $<0.0008$ & $<0.0009$ & $<0.0010$ & $<0.0009$ & 0.0001 \\
\hline \multicolumn{5}{|l|}{ Nickel } & \multicolumn{2}{|c|}{ Nickel } \\
\hline Impurity content detected by acid leach (ppmw) & $<0.0046$ & $<0.0045$ & $<0.0042$ & $<0.0048$ & $<0.0045$ & 0.0002 \\
\hline Impurity content detected in ash (ppmw) & $<0.0006$ & $<0.0006$ & $<0.0006$ & $<0.0006$ & $<0.0006$ & 0.0000 \\
\hline Total impurity content in ring blanks (ppmw) & $<0.0051$ & $<0.0051$ & $<0.0048$ & $<0.0054$ & $<0.0051$ & 0.0002 \\
\hline \multicolumn{5}{|l|}{ Calcium } & \multicolumn{2}{|c|}{ Calcium } \\
\hline Impurity content detected by acid leach (ppmw) & 3.574 & 3.483 & 3.731 & 4.188 & 3.7 & 0.3 \\
\hline Impurity content detected in ash (ppmw) & 0.133 & 0.138 & 0.133 & 0.146 & 0.138 & 0.006 \\
\hline Total impurity content in ring blanks (ppmw) & 3.706 & 3.621 & 3.864 & 4.334 & 3.9 & 0.3 \\
\hline \multicolumn{5}{|l|}{ Aluminum } & \multicolumn{2}{|c|}{ Aluminum } \\
\hline Impurity content detected by acid leach (ppmw) & 1.218 & 1.168 & 0.934 & 1.134 & 1.11 & 0.12 \\
\hline Impurity content detected in ash (ppmw) & 0.212 & 0.242 & 0.206 & 0.204 & 0.216 & 0.018 \\
\hline Total impurity content in ring blanks (ppmw) & 1.430 & 1.410 & 1.140 & 1.338 & 1.33 & 0.13 \\
\hline \multicolumn{5}{|l|}{ Titanium } & \multicolumn{2}{|c|}{ Titanium } \\
\hline Impurity content detected by acid leach (ppmw) & 0.567 & 0.551 & 0.576 & 0.614 & 0.58 & 0.03 \\
\hline Impurity content detected in ash (ppmw) & 0.311 & 0.356 & 0.190 & 0.287 & 0.29 & 0.07 \\
\hline Total impurity content in ring blanks (ppmw) & 0.878 & 0.907 & 0.766 & 0.901 & 0.86 & 0.07 \\
\hline \multicolumn{5}{|l|}{ Vanadium } & \multicolumn{2}{|c|}{ Vanadium } \\
\hline Impurity content detected by acid leach (ppmw) & 1.149 & 1.164 & 1.152 & 1.215 & 1.17 & 0.03 \\
\hline Impurity content in residual ash (ppmw) & 0.0239 & 0.0254 & 0.0239 & 0.0223 & 0.0239 & 0.0013 \\
\hline Total impurity content in ring blanks (ppmw) & 1.172 & 1.190 & 1.176 & 1.238 & 1.19 & 0.03 \\
\hline \multicolumn{5}{|l|}{\begin{tabular}{|l} 
Titanium plus Vanadium \\
\end{tabular}} & \multicolumn{2}{|c|}{ Titanium plus Vanadium } \\
\hline Impurity content detected by acid leach (ppmw) & 1.715 & 1.716 & 1.728 & 1.829 & 1.75 & 0.05 \\
\hline Impurity content detected in ash (ppmw) & 0.335 & 0.381 & 0.214 & 0.309 & 0.31 & 0.07 \\
\hline Total impurity content in ring blanks (ppmw) & 2.051 & 2.097 & 1.943 & 2.138 & 2.06 & 0.08 \\
\hline
\end{tabular}

Comments

Total impurity content in this table is the sum of the impurities detected by the standard burn-leach procedure plus the impurities detected by microwave digestion of the ash residue remaining after the standard burn-leach in nitric acid. Most of the residual ash was consumed by the microwave digestion. In addition to the impurities reported in the table, appreciable amounts of Si and Ba were also detected in the ash.
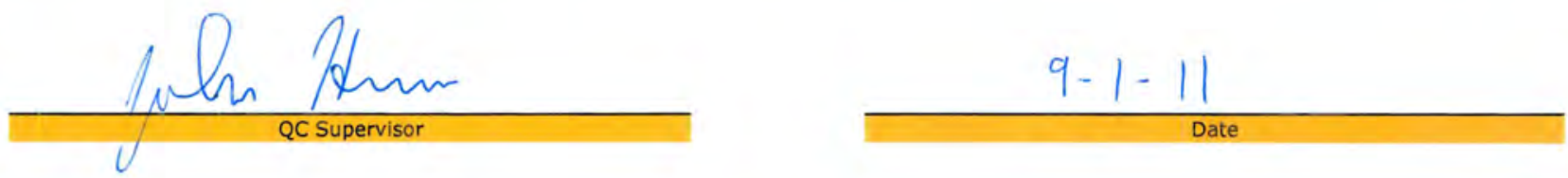


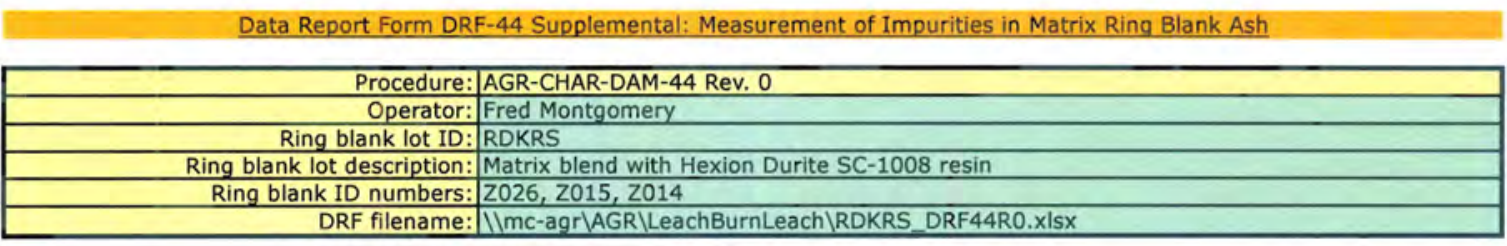

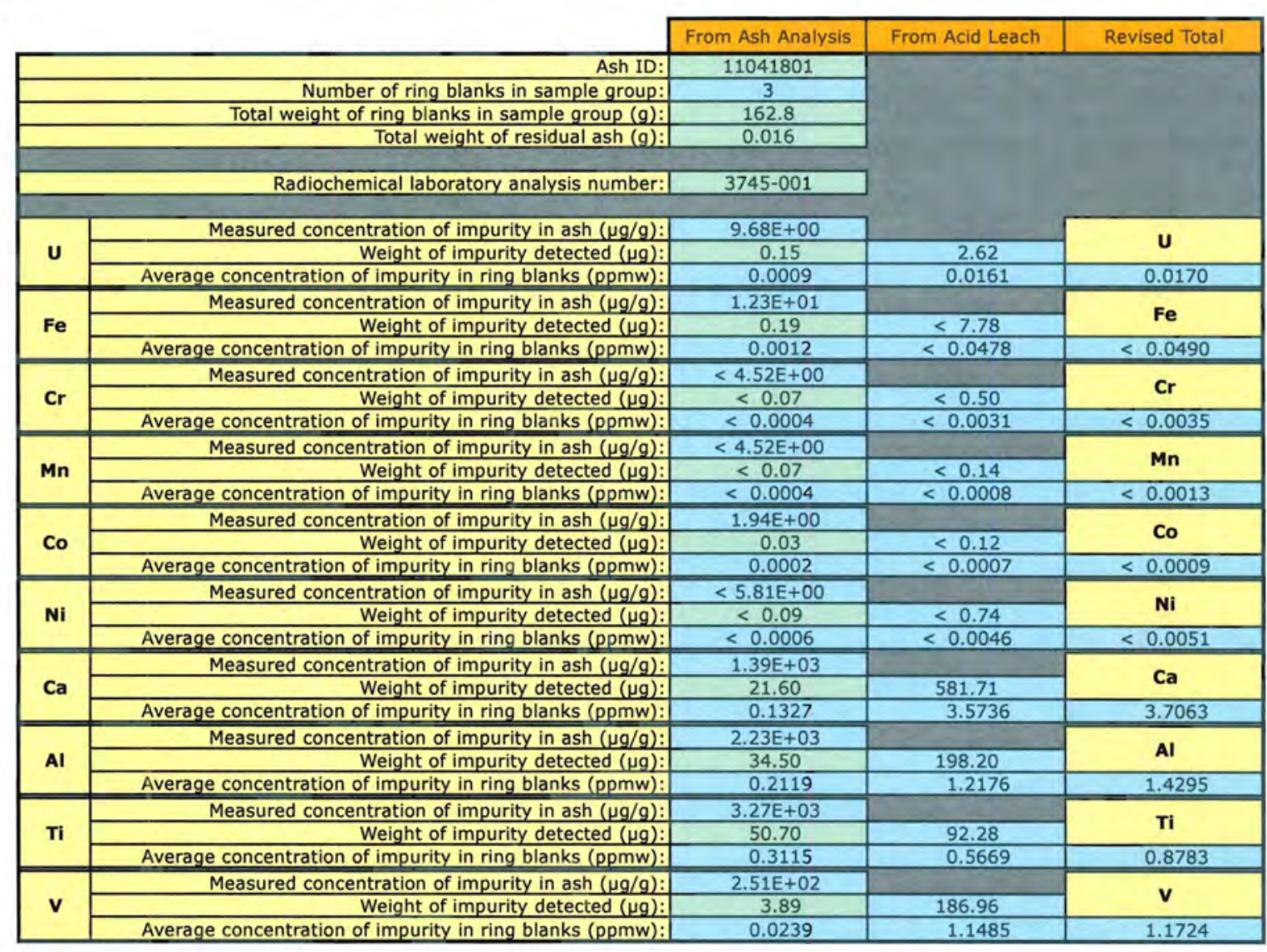

\section{Comments}

Additional ring blank impurities greater than about 1 ppmw detected in ash: 93 ppmw $\mathrm{Si}_{1} 1$ ppmw Ba.

Data checked by FCM against the official results of analyses for RMAL3745 on 8/19/2011.

\section{Fiederiek C. Mentgomeey}

$9-1-2011$ 
Data Report Form DRF-44 Supplemental: Measurement of Impurities in Matrix Ring Blank Ash

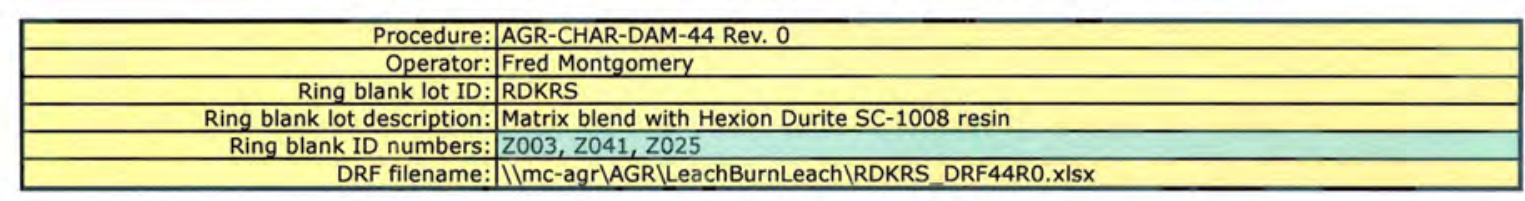

\begin{tabular}{|c|c|c|c|c|}
\hline \multirow{2}{*}{\multicolumn{2}{|c|}{ Ash ID: }} & From Ash Analysis & From Acid Leach & \multirow[t]{2}{*}{ Revised Total } \\
\hline & & 11041802 & & \\
\hline \multicolumn{2}{|r|}{ Number of ring blanks in sample group: } & 3 & & \\
\hline \multirow{2}{*}{\multicolumn{2}{|c|}{$\begin{array}{r}\text { Total weight of ring blanks in sample group }(\mathrm{g}) \text { : } \\
\text { Total weight of residual ash }(\mathrm{g}) \text { : }\end{array}$}} & 162.4 & & \\
\hline & & 0.018 & & \\
\hline \multicolumn{2}{|r|}{ Radiochemical laboratory analysis number: } & $3745-002$ & & \multirow{3}{*}{$\mathbf{u}$} \\
\hline \multirow{3}{*}{$\mathbf{u}$} & Measured concentration of impurity in ash $(\mu \mathrm{g} / \mathrm{g})$ : & $1.24 \mathrm{E}+01$ & & \\
\hline & Weight of impurity detected $(\mu \mathrm{g})$ : & 0.22 & 3.21 & \\
\hline & Average concentration of impurity in ring blanks (ppmw): & 0.0014 & 0.0198 & 0.0211 \\
\hline \multirow{3}{*}{$\mathbf{F e}$} & Measured concentration of impurity in ash $(\mu \mathrm{g} / \mathrm{g})$ : & $1.24 E+01$ & & \multirow{2}{*}{$\mathrm{Fe}$} \\
\hline & Weight of impurity detected $(\mu \mathrm{g})$ : & 0.22 & $<8.47$ & \\
\hline & Average concentration of impurity in ring blanks (ppmw): & 0.0014 & $<0.0521$ & $<0.0535$ \\
\hline \multirow{3}{*}{$\mathrm{Cr}$} & Measured concentration of impurity in ash $(\mu \mathrm{g} / \mathrm{g})$ : & $<4.49 E+00$ & & \multirow[b]{2}{*}{$\mathbf{C r}$} \\
\hline & Weight of impurity detected $(\mu \mathrm{g}):$ & $<0.08$ & $<0.55$ & \\
\hline & Average concentration of impurity in ring blanks (ppmw): & $<0.0005$ & $<0.0034$ & $<0.0039$ \\
\hline \multirow{3}{*}{ Mn } & Measured concentration of impurity in ash $(\mu \mathrm{g} / \mathrm{g})$ : & $<4.49 \mathrm{E}+00$ & & \multirow{2}{*}{ Mn } \\
\hline & Weight of impurity detected $(\mu g):$ & $<0.08$ & $<0.13$ & \\
\hline & Average concentration of impurity in ring blanks (ppmw): & $<0.0005$ & $<0.0008$ & $<0.0013$ \\
\hline \multirow{3}{*}{ Co } & Measured concentration of impurity in ash $(\mu \mathrm{g} / \mathrm{g})$ : & $<1.69 \mathrm{E}+00$ & & \multirow{2}{*}{ Co } \\
\hline & Weight of impurity detected $(\mu \mathrm{g}):$ & $<0.03$ & $<0.11$ & \\
\hline & Average concentration of impurity in ring blanks (ppmw): & $<0.0002$ & $<0.0007$ & $<0.0008$ \\
\hline \multirow{3}{*}{$\mathbf{N i}$} & Measured concentration of impurity in ash $(\mu \mathrm{g} / \mathrm{g})$ : & $<5.62 E+00$ & & \multirow{2}{*}{ Ni } \\
\hline & Weight of impurity detected $(\mu \mathrm{g})$ : & $<0.10$ & $<0.72$ & \\
\hline & Average concentration of impurity in ring blanks (ppmw): & $<0.0006$ & $<0.0045$ & $<0.0051$ \\
\hline \multirow{3}{*}{$\mathbf{C a}$} & Measured concentration of impurity in ash $(\mu \mathrm{g} / \mathrm{g})$ : & $1.26 \mathrm{E}+03$ & & \multirow[b]{2}{*}{$\mathbf{C a}$} \\
\hline & Weight of impurity detected $(\mu \mathrm{g}):$ & 22.40 & 565.51 & \\
\hline & Average concentration of impurity in ring blanks (ppmw): & 0.1380 & 3.4829 & 3.6208 \\
\hline \multirow{3}{*}{ Al } & Measured concentration of impurity in ash $(\mu \mathrm{g} / \mathrm{g})$ : & $2.21 \mathrm{E}+03$ & & \multirow{2}{*}{ Al } \\
\hline & Weight of impurity detected $(\mu g)$ : & 39.30 & 189.64 & \\
\hline & Average concentration of impurity in ring blanks (ppmw): & 0.2420 & 1.1680 & 1.4100 \\
\hline \multirow{3}{*}{$\mathbf{T i}$} & Measured concentration of impurity in ash $(\mu \mathrm{g} / \mathrm{g})$ : & $3.25 E+03$ & & \multirow{2}{*}{$\mathrm{Ti}$} \\
\hline & Weight of impurity detected $(\mu \mathrm{g})$ : & 57.80 & 89.54 & \\
\hline & Average concentration of impurity in ring blanks (ppmw): & 0.3560 & 0.5515 & 0.9075 \\
\hline \multirow{3}{*}{$\mathbf{v}$} & Measured concentration of impurity in ash $(\mu \mathrm{g} / \mathrm{g})$ : & $2.32 E+02$ & & \multirow{2}{*}{$\mathbf{v}$} \\
\hline & Weight of impurity detected $(\mu \mathrm{g})$ : & 4.13 & 189.04 & \\
\hline & Average concentration of impurity in ring blanks (ppmw): & 0.0254 & 1.1643 & 1.1897 \\
\hline
\end{tabular}

\section{Comments}

Additional ring blank impurities greater than about 1 ppmw detected in ash: $106 \mathrm{ppmw} \mathrm{Si,} 3$ ppmw Ba. Data checked by FCM against the official results of analyses for RMAL3745 on 8/19/2011.

Fredeuck C. Montgomery
$9-1-2011$ Date 


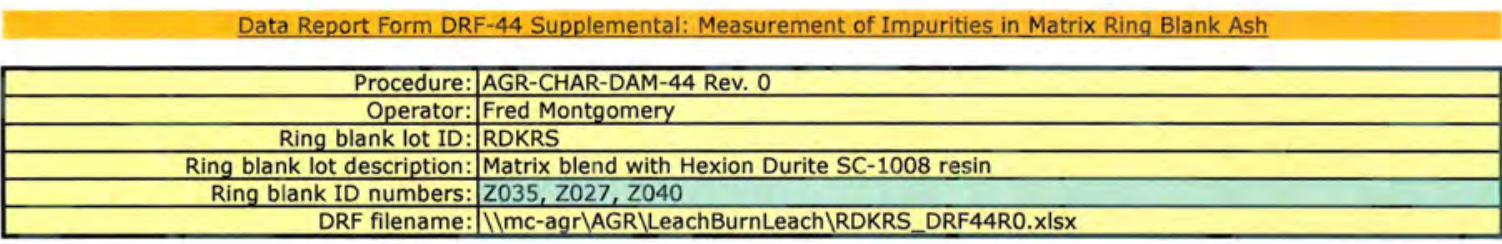

\begin{tabular}{|c|c|c|c|c|}
\hline \multirow{2}{*}{\multicolumn{2}{|c|}{ Ash ID: }} & From Ash Analysis & From Acid Leach & Revised Total \\
\hline & & 11041803 & & \\
\hline \multicolumn{2}{|r|}{ Number of ring blanks in sample group: } & 3 & & \\
\hline \multirow{2}{*}{\multicolumn{2}{|c|}{$\begin{array}{r}\text { Total weight of ring blanks in sample group }(\mathrm{g}) \text { : } \\
\text { Total weight of residual ash }(\mathrm{g}) \text { : }\end{array}$}} & 162.5 & & \\
\hline & & 0.018 & & \\
\hline \multicolumn{2}{|r|}{ Radiochemical laboratory analysis number: } & $3745-003$ & & \\
\hline \multirow{3}{*}{$\mathbf{u}$} & Measured concentration of impurity in ash $(\mu \mathrm{g} / \mathrm{g})$ : & $1.33 E+01$ & & \multirow[b]{2}{*}{$\mathbf{u}$} \\
\hline & Weight of impurity detected $(\mu \mathrm{g})$ : & 0.24 & 2.23 & \\
\hline & Average concentration of impurity in ring blanks (ppmw): & 0.0015 & 0.0137 & 0.0152 \\
\hline \multirow{3}{*}{$\mathbf{F e}$} & Measured concentration of impurity in ash $(\mu \mathrm{g} / \mathrm{g})$ : & $1.10 E+02$ & & \multirow{2}{*}{$\mathrm{Fe}$} \\
\hline & Weight of impurity detected $(\mu \mathrm{g})$ : & 2.00 & $<6.60$ & \\
\hline & Average concentration of impurity in ring blanks (ppmw): & 0.0123 & $<0.0406$ & $<0.0529$ \\
\hline \multirow{3}{*}{$\mathbf{C r}$} & Measured concentration of impurity in ash $(\mu \mathrm{g} / \mathrm{g})$ : & $<4.42 E+00$ & & \multirow{2}{*}{ Cr } \\
\hline & Weight of impurity detected $(\mu \mathrm{g})$ : & $<0.08$ & $<0.42$ & \\
\hline & Average concentration of impurity in ring blanks (ppmw): & $<0.0005$ & $<0.0026$ & $<0.0031$ \\
\hline \multirow{3}{*}{ Mn } & Measured concentration of impurity in ash $(\mu \mathrm{g} / \mathrm{g})$ : & $<4.42 E+00$ & & \multirow{2}{*}{ Mn } \\
\hline & Weight of impurity detected $(\mu \mathrm{g})$ : & $<0.08$ & $<0.14$ & \\
\hline & Average concentration of impurity in ring blanks (ppmw): & $<0.0005$ & $<0.0009$ & $<0.0014$ \\
\hline \multirow{3}{*}{ Co } & Measured concentration of impurity in ash $(\mu g / g)$ : & $<1.66 \mathrm{E}+00$ & & \multirow[b]{2}{*}{ Co } \\
\hline & Weight of impurity detected $(\mu \mathrm{g})$ : & $<0.03$ & $<0.12$ & \\
\hline & Average concentration of impurity in ring blanks (ppmw): & $<0.0002$ & $<0.0007$ & $<0.0009$ \\
\hline \multirow{3}{*}{$\mathbf{N i}$} & Measured concentration of impurity in ash $(\mu \mathrm{g} / \mathrm{g})$ : & $<5.52 E+00$ & & \multirow{2}{*}{$\mathbf{N i}$} \\
\hline & Weight of impurity detected $(\mu \mathrm{g})$ : & $<0.10$ & $<0.69$ & \\
\hline & Average concentration of impurity in ring blanks (ppmw): & $<0.0006$ & $<0.0042$ & $<0.0048$ \\
\hline \multirow{3}{*}{$\mathbf{C a}$} & Measured concentration of impurity in ash $(\mu \mathrm{g} / \mathrm{g})$ : & $1.19 \mathrm{E}+03$ & & \multirow[b]{2}{*}{ Ca } \\
\hline & Weight of impurity detected $(\mu \mathrm{g})$ : & 21.60 & 606.28 & \\
\hline & Average concentration of impurity in ring blanks (ppmw): & 0.1329 & 3.7314 & 3.8644 \\
\hline \multirow{3}{*}{ Al } & Measured concentration of impurity in ash $(\mu \mathrm{g} / \mathrm{g})$ : & $1.85 E+03$ & & \multirow[b]{2}{*}{ Al } \\
\hline & Weight of impurity detected $(\mu g)$ : & 33.50 & 151.73 & \\
\hline & Average concentration of impurity in ring blanks (ppmw): & 0.2062 & 0.9338 & 1.1400 \\
\hline \multirow{3}{*}{$\mathbf{T i}$} & Measured concentration of impurity in ash $(\mu \mathrm{g} / \mathrm{g})$ : & $1.71 \mathrm{E}+03$ & & \multirow{2}{*}{ Ti } \\
\hline & Weight of impurity detected $(\mu \mathrm{g})$ : & 30.90 & 93.58 & \\
\hline & Average concentration of impurity in ring blanks (ppmw): & 0.1902 & 0.5759 & 0.7661 \\
\hline \multirow{3}{*}{$\mathbf{v}$} & Measured concentration of impurity in ash $(\mu \mathrm{g} / \mathrm{g})$ : & $2.15 E+02$ & & \multirow{2}{*}{$\mathbf{v}$} \\
\hline & Weight of impurity detected $(\mu \mathrm{g})$ : & 3.89 & 187.26 & \\
\hline & Average concentration of impurity in ring blanks (ppmw): & 0.0239 & 1.1525 & 1.1764 \\
\hline
\end{tabular}


Data Report Form DRF-44 Supplemental: Measurement of Impurities in Matrix Rina Blank Ash

\begin{tabular}{|c|c|}
\hline Procedure: & AGR-CHAR-DAM-44 Rev. 0 \\
\hline Operator: & Fred Montgomery \\
\hline Ring blank lot ID: & RDKRS \\
\hline Ring blank lot description: & Matrix blend with Hexion Durite SC- 1008 resin \\
\hline Ring blank ID numbers: & Z019, Z036, Z044 \\
\hline DRF filename: & 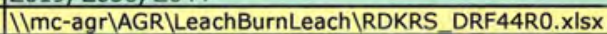 \\
\hline
\end{tabular}

\begin{tabular}{|c|c|c|c|c|}
\hline & \multirow[b]{2}{*}{ Ash ID: } & From Ash Analysis & \multirow[t]{2}{*}{ From Acid Leach } & Revised Total \\
\hline & & 11041804 & & \\
\hline & Number of ring blanks in sample group: & 3 & & \\
\hline & Total weight of ring blanks in sample group $(g)$ : & 162.5 & & \\
\hline & Total weight of residual ash $(g):$ & 0.017 & & \\
\hline & Radiochemical laboratory analysis number: & $3745-004$ & & \\
\hline & Measured concentration of impurity in ash $(\mu \mathrm{g} / \mathrm{g})$ : & $7.60 E+00$ & & \\
\hline $\mathbf{u}$ & Weight of impurity detected $(\mu \mathrm{g}):$ & 0.13 & 2.23 & u \\
\hline & Average concentration of impurity in ring blanks (ppmw): & 0.0008 & 0.0137 & 0.0145 \\
\hline & Measured concentration of impurity in ash $(\mu \mathrm{g} / \mathrm{g})$ : & $2.18 \mathrm{E}+02$ & & \\
\hline $\mathbf{F e}$ & Weight of impurity detected $(\mu \mathrm{g})$ : & 3.73 & 16.47 & $\mathbf{F e}$ \\
\hline & Average concentration of impurity in ring blanks (ppmw): & 0.0230 & 0.1014 & 0.1243 \\
\hline & Measured concentration of impurity in ash $(\mu \mathrm{g} / \mathrm{g})$ : & $<4.68 \mathrm{E}+00$ & & $\mathrm{Cr}_{\mathrm{r}}$ \\
\hline $\mathbf{C r}$ & Weight of impurity detected $(\mu \mathrm{g})$ : & $<0.08$ & $<0.48$ & $\mathrm{Cr}$ \\
\hline & Average concentration of impurity in ring blanks (ppmw): & $<0.0005$ & $<0.0029$ & $<0.0034$ \\
\hline & Measured concentration of impurity in ash $(\mu \mathrm{g} / \mathrm{g})$ : & $<4.68 \mathrm{E}+00$ & & \\
\hline Mn & Weight of impurity detected $(\mu \mathrm{g})$ : & $<0.08$ & $<0.16$ & Mn \\
\hline & Average concentration of impurity in ring blanks (ppmw): & $<0.0005$ & $<0.0010$ & $<0.0015$ \\
\hline & Measured concentration of impurity in ash $(\mu \mathrm{g} / \mathrm{g})$ : & $<1.75 E+00$ & & \\
\hline Co & Weight of impurity detected $(\mu \mathrm{g})$ : & $<0.03$ & $<0.13$ & Co \\
\hline & Average concentration of impurity in ring blanks (ppmw): & $<0.0002$ & $<0.0008$ & $<0.0010$ \\
\hline & Measured concentration of impurity in ash $(\mu \mathrm{g} / \mathrm{g})$ : & $<5.85 E+00$ & & \\
\hline $\mathbf{N i}$ & Weight of impurity detected $(\mu \mathrm{g})$ : & $<0.10$ & $<0.77$ & $\mathbf{N i}$ \\
\hline & Average concentration of impurity in ring blanks (ppmw): & $<0.0006$ & $<0.0048$ & $<0.0054$ \\
\hline & Measured concentration of impurity in ash $(\mu \mathrm{g} / \mathrm{g})$ : & $1.39 \mathrm{E}+03$ & & C \\
\hline $\mathrm{Ca}$ & Weight of impurity detected $(\mu \mathrm{g})$ : & 23.80 & 680.56 & Ca \\
\hline & Average concentration of impurity in ring blanks (ppmw): & 0.1464 & 4.1876 & 4.3341 \\
\hline & Measured concentration of impurity in ash $(\mu \mathrm{g} / \mathrm{g})$ : & $1.94 \mathrm{E}+03$ & & Al \\
\hline Al & Weight of impurity detected $(\mu \mathrm{g})$ : & 33.10 & 184.34 & Al \\
\hline & Average concentration of impurity in ring blanks (ppmw): & 0.2037 & 1.1343 & 1.3380 \\
\hline & Measured concentration of impurity in ash $(\mu \mathrm{g} / \mathrm{g})$ : & $2.73 E+03$ & & \\
\hline $\mathbf{T i}$ & Weight of impurity detected $(\mu \mathrm{g})$ : & 46.60 & 99.76 & $\mathrm{Ti}$ \\
\hline & Average concentration of impurity in ring blanks (ppmw): & 0.2867 & 0.6139 & 0.9006 \\
\hline & Measured concentration of impurity in ash $(\mu \mathrm{g} / \mathrm{g})$ : & $2.12 \mathrm{E}+02$ & & \\
\hline v & Weight of impurity detected $(\mu \mathrm{g})$ : & 3.62 & 197.50 & $\mathbf{v}$ \\
\hline & Average concentration of impurity in ring blanks (ppmw): & 0.0223 & 1.2153 & 1.2376 \\
\hline
\end{tabular}

\section{Comments}

Additional ring blank impurities greater than about 1 ppmw detected in ash: 102 ppmw Si, 2 ppmw Ba. Data checked by FCM against the official results of analyses for RMAL3745 on 8/19/2011.

Fuderick C. Montgomely
$9-(-201)$ 


\section{Analysis of Impurity Content in Machined Matrix Rings}

Of the 30 ring blanks sent to INL (Table 3), 6 were returned to ORNL after machining for analysis of impurity content. The $26 \mathrm{~mm}$ diameter, $63 \mathrm{~mm}$ long solid cylindrical ring blanks were machined down to approximately $24.4 \mathrm{~mm}$ diameter, $50.8 \mathrm{~mm}$ long cylinders. An approximately $12.5 \mathrm{~mm}$ diameter hole was drilled down the axis to receive the fuel compacts. Thermocouple and melt wire cylindrical wells were also drilled into the shoulder of the rings.

Machined matrix rings were burned in air and the ash was leached twice by boiling nitric acid. Ash residue remaining after the standard burn-leach analysis was analyzed by ICP-MS after microwave digestion. The analysis was performed on the 6 machined matrix rings, in three sample groups with 2 rings in each sample group. The following pages are copies of the inspection report form IRF-20B, which summarizes the results of the burn-leach analysis, and IRF-20B Supplemental, which adds data from microwave digestion of the residual ash to the results of the burn-leach analysis. Following the inspection report forms are the individual data report forms for the measurements that were performed.

Table 6 compares the results of the machined matrix ring impurity analysis to the impurity analysis of the ring blanks reported in Section 4 and Section 6. The values reported in Table 6 include data from both burn-leach of the machined matrix rings and subsequent microwave digestion of the ash residue. There was no significant change in the uranium content. Other impurities showed a small but measurable increase, which could be related to contamination from the machining or extra handling. All of these values are still well below the specified limits, so the additional contamination should not present an issue.

Table 6. Impurity content (ppmw) before and after machining

\begin{tabular}{|l|c|c|c|c|c|}
\hline \multirow{2}{*}{ Specified Impurity } & \multirow{2}{*}{$\begin{array}{c}\text { Upper Limit at } \\
\text { 95\% confidence }\end{array}$} & \multicolumn{2}{c|}{$\begin{array}{c}\text { RDRS Ring } \\
\text { Blanks }\end{array}$} & \multicolumn{2}{c|}{$\begin{array}{c}\text { Machined Matrix } \\
\text { Rings }\end{array}$} \\
\cline { 3 - 6 } & & mean & std. dev. & mean & std. dev. \\
\hline Uranium & $\leq 0.5$ & 0.017 & 0.003 & 0.013 & 0.005 \\
\hline Iron & $\leq 20$ & $<0.07$ & 0.04 & $<0.44$ & 0.18 \\
\hline Chromium & $\leq 10$ & $<0.0035$ & 0.0003 & $<0.035$ & 0.011 \\
\hline Manganese & $\leq 10$ & $<0.0013$ & 0.0001 & $<0.0059$ & 0.0009 \\
\hline Cobalt & $\leq 10$ & $<0.0009$ & 0.0001 & $<0.0057$ & 0.0016 \\
\hline Nickel & $\leq 10$ & $<0.0051$ & 0.0002 & $<0.025$ & 0.002 \\
\hline Calcium & $\leq 45$ & 3.9 & 0.3 & 5.6 & 0.9 \\
\hline Aluminum & $\leq 20$ & 1.33 & 0.13 & 2.4 & 0.3 \\
\hline Titanium & NA & 0.86 & 0.07 & 1.2 & 0.2 \\
\hline Vanadium & NA & 1.19 & 0.03 & 1.82 & 0.11 \\
\hline Titanium plus Vanadium & $\leq 85$ & 2.06 & 0.08 & 3.0 & 0.3 \\
\hline
\end{tabular}




\section{Inspection Report Form IRF-20B: Summary of Impurities in Matrix Rings}

\begin{tabular}{|r|l|}
\hline Procedure: & AGR-CHAR-PIP-20 Rev. 1 \\
\hline Operator: & Fred Montgomery \\
\hline Ring blank lot ID: & RDKRS-Machined \\
\hline Ring blank lot description: & Rings machined from blanks made using matrix blend with Hexion Durite SC-1008 resin \\
\hline
\end{tabular}

\begin{tabular}{|c|c|c|c|c|c|}
\hline Ring blank ID numbers: & zoos, z010 & 2022,2023 & Z037, Z039 & Mean & $\begin{array}{l}\text { Standard } \\
\text { Deviation }\end{array}$ \\
\hline \multicolumn{4}{|l|}{ Uranium } & \multicolumn{2}{|c|}{ Uranium } \\
\hline Average impurity content in ring blanks (ppmw) & $<0.009$ & 0.009 & 0.018 & $<0.012$ & 0.005 \\
\hline \multicolumn{4}{|l|}{ Iron } & \multicolumn{2}{|c|}{ Iron } \\
\hline Average impurity content in ring blanks (ppmw) & $<0.287$ & 0.602 & 0.355 & $<0.41$ & 0.17 \\
\hline \multicolumn{4}{|l|}{ Chromium } & \multicolumn{2}{|c|}{ Chromium } \\
\hline Average impurity content in ring blanks (ppmw) & $<0.026$ & 0.045 & 0.030 & $<0.033$ & 0.010 \\
\hline \multicolumn{4}{|l|}{ Manganese } & \multicolumn{2}{|c|}{ Manganese } \\
\hline Average impurity content in ring blanks (ppmw) & $<0.0044$ & 0.0061 & 0.0048 & $<0.0051$ & 0.0009 \\
\hline \multicolumn{4}{|l|}{ Cobalt } & \multicolumn{2}{|c|}{ Cobalt } \\
\hline Average impurity content in ring blanks (ppmw) & $<0.0041$ & 0.0068 & 0.0042 & $<0.0050$ & 0.0016 \\
\hline \multicolumn{4}{|l|}{ Nickel } & \multicolumn{2}{|c|}{ Nickel } \\
\hline Average impurity content in ring blanks (ppmw) & $<0.0199$ & 0.0239 & 0.0195 & $<0.021$ & 0.002 \\
\hline \multicolumn{4}{|l|}{ Calcium } & \multicolumn{2}{|c|}{ Calcium } \\
\hline Average impurity content in ring blanks (ppmw) & 5.576 & 4.528 & 6.277 & 5.5 & 0.9 \\
\hline \multicolumn{4}{|l|}{ Aluminum } & \multicolumn{2}{|c|}{ Aluminum } \\
\hline Average impurity content in ring blanks (ppmw) & 1.843 & 2.527 & 1.927 & 2.1 & 0.4 \\
\hline \multicolumn{4}{|l|}{ Titanium } & \multicolumn{2}{|c|}{ Titanium } \\
\hline Average impurity content in ring blanks (ppmw) & 0.710 & 0.817 & 0.740 & 0.76 & 0.05 \\
\hline \multicolumn{4}{|l|}{ Vanadium } & \multicolumn{2}{|c|}{ Vanadium } \\
\hline Average impurity content in ring blanks (ppmw) & 1.739 & 1.928 & 1.725 & 1.80 & 0.11 \\
\hline \multicolumn{4}{|l|}{ Titanium plus Vanadium } & \multicolumn{2}{|c|}{ Titanium plus Vanadium } \\
\hline Average impurity content in ring blanks (ppmw) & 2.449 & 2.745 & 2.465 & 2.55 & 0.17 \\
\hline
\end{tabular}

Six ring blanks were machined by INL into matrix rings and returned to ORNL for analysis. After burning and leaching with nitric acid, a white ash remained in the leach vessel. These impurity analysis results do not include content of the ash. This ash was mostly silica.

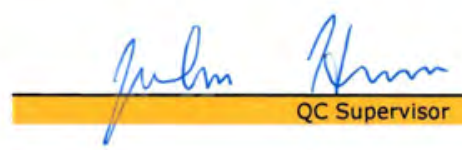

$$
9-7-11
$$


Inspection Report Form IRF-20B Supplemental: Summary of Impurities in Matrix Rings Including Analysis of Ash Residue

\begin{tabular}{|r|l|}
\hline Procedure: & AGR-CHAR-PIP-20 Rev. 1 \\
\hline Operator: & Fred Montgomery \\
\hline Ring blank lot ID: & RDKRS-Machined \\
\hline Ring blank lot description: & Rings machined from blanks made using matrix blend with Hexion Durite SC-1008 resin \\
\hline
\end{tabular}

\begin{tabular}{|c|c|c|c|c|c|}
\hline Ring blank ID numbers: & Z005, Z010 & Z022, Z023 & Z037, Z039 & Mean & $\begin{array}{l}\text { Standard } \\
\text { Deviation }\end{array}$ \\
\hline \multicolumn{4}{|l|}{ Uranium } & \multicolumn{2}{|c|}{ Uranium } \\
\hline Impurity content detected by acid leach (ppmw) & $<0.009$ & 0.009 & 0.018 & $<0.012$ & 0.005 \\
\hline Impurity content detected in ash (ppmw) & 0.0009 & 0.0008 & 0.0014 & 0.0010 & 0.0003 \\
\hline Total impurity content in ring blanks (ppmw) & 0.010 & 0.010 & 0.019 & 0.013 & 0.005 \\
\hline \multicolumn{4}{|l|}{ Iron } & \multicolumn{2}{|c|}{ Iron } \\
\hline Impurity content detected by acid leach (ppmw) & $<0.287$ & 0.602 & 0.355 & $<0.41$ & 0.17 \\
\hline Impurity content detected in ash (ppmw) & $<0.018$ & 0.041 & $<0.018$ & $<0.026$ & 0.013 \\
\hline Total impurity content in ring blanks (ppmw) & $<0.305$ & 0.643 & $<0.373$ & $<0.44$ & 0.18 \\
\hline \multicolumn{4}{|l|}{ Chromium } & \multicolumn{2}{|c|}{ Chromium } \\
\hline Impurity content detected by acid leach (ppmw) & $<0.026$ & 0.045 & 0.030 & $<0.033$ & 0.010 \\
\hline Impurity content detected in ash (ppmw) & 0.0010 & 0.0029 & 0.0017 & 0.0019 & 0.0010 \\
\hline Total impurity content in ring blanks (ppmw) & $<0.027$ & 0.048 & 0.032 & $<0.035$ & 0.011 \\
\hline \multicolumn{4}{|l|}{ Manganese } & \multicolumn{2}{|c|}{ Manganese } \\
\hline Impurity content detected by acid leach (ppmw) & $<0.0044$ & 0.0061 & 0.0048 & $<0.0051$ & 0.0009 \\
\hline Impurity content detected in ash (ppmw) & $<0.0008$ & $<0.0008$ & $<0.0008$ & $<0.0008$ & 0.0000 \\
\hline Total impurity content in ring blanks (ppmw) & $<0.0052$ & $<0.0070$ & $<0.0056$ & $<0.0059$ & 0.0009 \\
\hline \multicolumn{4}{|l|}{ Cobalt } & \multicolumn{2}{|c|}{ Cobalt } \\
\hline Impurity content detected by acid leach (ppmw) & $<0.0041$ & 0.0068 & 0.0042 & $<0.0050$ & 0.0016 \\
\hline Impurity content detected in ash (ppmw) & $<0.0007$ & $<0.0007$ & $<0.0007$ & $<0.0007$ & 0.0000 \\
\hline Total impurity content in ring blanks (ppmw) & $<0.0048$ & $<0.0075$ & $<0.0049$ & $<0.0057$ & 0.0016 \\
\hline \multicolumn{4}{|l|}{ Nickel } & \multicolumn{2}{|c|}{ Nickel } \\
\hline Impurity content detected by acid leach (ppmw) & $<0.0199$ & 0.0239 & 0.0195 & $<0.021$ & 0.002 \\
\hline Impurity content detected in ash (ppmw) & $<0.0036$ & $<0.0035$ & $<0.0035$ & $<0.0035$ & 0.0000 \\
\hline Total impurity content in ring blanks (ppmw) & $<0.0235$ & $<0.0274$ & $<0.0230$ & $<0.025$ & 0.002 \\
\hline \multicolumn{4}{|l|}{ Calcium } & \multicolumn{2}{|c|}{ Calcium } \\
\hline Impurity content detected by acid leach (ppmw) & 5.576 & 4.528 & 6.277 & 5.5 & 0.9 \\
\hline Impurity content detected in ash (ppmw) & 0.146 & 0.112 & 0.097 & 0.12 & 0.03 \\
\hline Total impurity content in ring blanks (ppmw) & 5.722 & 4.640 & 6.374 & 5.6 & 0.9 \\
\hline \multicolumn{4}{|l|}{ Aluminum } & \multicolumn{2}{|c|}{ Aluminum } \\
\hline Impurity content detected by acid leach (ppmw) & 1.843 & 2.527 & 1.927 & 2.1 & 0.4 \\
\hline Impurity content detected in ash (ppmw) & 0.372 & 0.301 & 0.328 & 0.33 & 0.04 \\
\hline Total impurity content in ring blanks (ppmw) & 2.216 & 2.829 & 2.254 & 2.4 & 0.3 \\
\hline \multicolumn{4}{|l|}{ Titanium } & \multicolumn{2}{|c|}{ Titanium } \\
\hline Impurity content detected by acid leach (ppmw) & 0.710 & 0.817 & 0.740 & 0.76 & 0.05 \\
\hline Impurity content detected in ash (ppmw) & 0.655 & 0.492 & 0.210 & 0.5 & 0.2 \\
\hline Total impurity content in ring blanks (ppmw) & 1.365 & 1.309 & 0.950 & 1.2 & 0.2 \\
\hline \multicolumn{4}{|l|}{ Vanadium } & \multicolumn{2}{|c|}{ Vanadium } \\
\hline Impurity content detected by acid leach (ppmw) & 1.739 & 1.928 & 1.725 & 1.80 & 0.11 \\
\hline Impurity content in residual ash (ppmw) & 0.031 & 0.026 & 0.020 & 0.026 & 0.005 \\
\hline Total impurity content in ring blanks (ppmw) & 1.770 & 1.954 & 1.745 & 1.82 & 0.11 \\
\hline \multicolumn{4}{|l|}{ Titanium plus Vanadium } & \multicolumn{2}{|c|}{ Titanium plus Vanadium } \\
\hline Impurity content detected by acid leach (ppmw) & 2.449 & 2.745 & 2.465 & 2.55 & 0.17 \\
\hline Impurity content detected in ash (ppmw) & 0.685 & 0.518 & 0.230 & 0.5 & 0.2 \\
\hline Total impurity content in ring blanks (ppmw) & 3.135 & 3.263 & 2.695 & 3.0 & 0.3 \\
\hline
\end{tabular}

Comments

Total impurity content in this table is the sum of the impurities detected by the standard burn-leach procedure plus the impurities detected by microwave digestion of the ash residue remaining after the standard burn-leach in nitric acid. Most of the residual ash was consumed by the microwave digestion. In addition to the impurities reported in the table, appreciable amounts of Si and Ba were also detected in the ash.

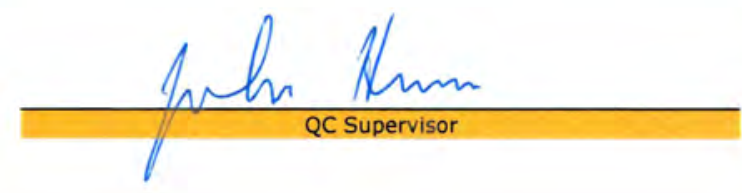

$$
9-7-11
$$


Data Report Form DRF-44: Measurement of Impurities in Matrix Ring Blanks by the Burn-Leach Method

\begin{tabular}{|c|c|}
\hline Procedure: & AGR-CHAR-DAM-44 Rev. 0 \\
\hline Operator: & Fred Montgomery \\
\hline Ring blank lot ID: & RDKRS-Machined \\
\hline Ring blank lot description: & Rings machined from blanks made using matrix blend with Hexion Durite SC- 1008 resin \\
\hline Ring ID numbers: & $\mathrm{Z} 005, \mathrm{Z} 010$ \\
\hline DRF filename: & \Imc-agr|AGR\LeachBurnLeach|RDKRS-Machined DRF44R0,x/s \\
\hline
\end{tabular}

\begin{tabular}{|c|c|c|c|c|}
\hline & & \\
\hline & & First Leach & Second Leach & Total \\
\hline & Leach solution ID: & BL11071501 & BL11071801 & \\
\hline & Number of ring blanks in sample group: & \multicolumn{2}{|l|}{50251202} & \\
\hline & Total weight of ring blanks in sample group $(\mathrm{g})$ : & \multicolumn{2}{|c|}{56.2} & \\
\hline & Total volume of leach solution $(\mathrm{ml}):$ & 39.9 & 38.0 & \\
\hline & Radiochemical laboratory analysis number: & $3864-001$ & $3864-005$ & \\
\hline & & & & \\
\hline \multirow{3}{*}{$\mathbf{u}$} & Measured concentration of impurity in solution $(\mu \mathrm{g} / \mathrm{ml})$ : & $1.23 E-02$ & $<2.00 \mathrm{E}-04$ & \multirow{2}{*}{$\mathbf{U}$} \\
\hline & Weight of impurity in sample group $(\mu \mathrm{g}):$ & 0.49 & $<0.01$ & \\
\hline & Average concentration of impurity in ring blanks (ppmw): & 0.0087 & $<0.0001$ & $<0.0089$ \\
\hline \multirow{3}{*}{$\mathbf{F e}$} & Measured concentration of impurity in solution $(\mu \mathrm{g} / \mathrm{ml})$ : & $3.64 \mathrm{E}-01$ & $<4.12 \mathrm{E}-02$ & \multirow{2}{*}{$\mathrm{Fe}$} \\
\hline & Weight of impurity in sample group $(\mu g)$ : & 14.52 & $<1.57$ & \\
\hline & Average concentration of impurity in ring blanks (ppmw): & 0.2586 & $<0.0279$ & $<0.2865$ \\
\hline \multirow{3}{*}{$\mathbf{C r}$} & Measured concentration of impurity in solution $(\mu \mathrm{g} / \mathrm{ml})$ : & $3.40 \mathrm{E}-02$ & $<2.00 \mathrm{E}-03$ & \multirow[b]{2}{*}{$\mathrm{Cr}$} \\
\hline & Weight of impurity in sample group $(\mu \mathrm{g})$ : & 1.36 & $<0.08$ & \\
\hline & Average concentration of impurity in ring blanks (ppmw): & 0.0242 & $<0.0014$ & $<0.0255$ \\
\hline \multirow{3}{*}{ Mn } & Measured concentration of impurity in solution $(\mu \mathrm{g} / \mathrm{ml})$ : & 4.33E-03 & $<1.91 \mathrm{E}-03$ & \multirow{2}{*}{ Mn } \\
\hline & Weight of impurity in sample group $(\mu g)$ : & 0.17 & $<0.07$ & \\
\hline & Average concentration of impurity in ring blanks (ppmw): & 0.0031 & $<0.0013$ & $<0.0044$ \\
\hline \multirow{3}{*}{ Co } & Measured concentration of impurity in solution $(\mu \mathrm{g} / \mathrm{ml})$ : & $4.19 E-03$ & $<1.62 \mathrm{E}-03$ & \multirow{2}{*}{ Co } \\
\hline & Weight of impurity in sample group $(\mu \mathrm{g})$ : & 0.17 & $<0.06$ & \\
\hline & Average concentration of impurity in ring blanks (ppmw): & 0.0030 & $<0.0011$ & $<0.0041$ \\
\hline \multirow{3}{*}{$\mathbf{N i}$} & Measured concentration of impurity in solution $(\mu \mathrm{g} / \mathrm{ml})$ : & 2.04E-02 & $<8.00 E-03$ & \multirow{2}{*}{$\mathrm{Ni}$} \\
\hline & Weight of impurity in sample group $(\mu g)$ : & 0.81 & $<0.30$ & \\
\hline & Average concentration of impurity in ring blanks (ppmw): & 0.0145 & $<0.0054$ & $<0.0199$ \\
\hline \multirow{3}{*}{$\mathbf{C a}$} & Measured concentration of impurity in solution $(\mu \mathrm{g} / \mathrm{ml})$ : & $7.35 E+00$ & $5.22 \mathrm{E}-01$ & \multirow{2}{*}{$\mathrm{Ca}$} \\
\hline & Weight of impurity in sample group $(\mu \mathrm{g})$ : & 293.27 & 19.84 & \\
\hline & Average concentration of impurity in ring blanks ( $\mathrm{ppmw}$ ): & 5.2223 & 0.3532 & 5.5756 \\
\hline \multirow{3}{*}{ Al } & Measured concentration of impurity in solution $(\mu \mathrm{g} / \mathrm{ml})$ : & $2.44 E+00$ & $1.62 \mathrm{E}-01$ & \multirow{2}{*}{ Al } \\
\hline & Weight of impurity in sample group $(\mu \mathrm{g})$ : & 97.36 & 6.16 & \\
\hline & Average concentration of impurity in ring blanks (ppmw): & 1.7337 & 0.1096 & 1.8433 \\
\hline \multirow{3}{*}{$\mathbf{T i}$} & Measured concentration of impurity in solution $(\mu \mathrm{g} / \mathrm{ml})$ : & $8.99 E-01$ & $1.06 \mathrm{E}-01$ & \multirow{2}{*}{$\mathrm{Ti}$} \\
\hline & Weight of impurity in sample group $(\mu \mathrm{g})$ : & 35.87 & 4.03 & \\
\hline & Average concentration of impurity in ring blanks (ppmw): & 0.6388 & 0.0717 & 0.7105 \\
\hline \multirow{3}{*}{$\mathbf{v}$} & Measured concentration of impurity in solution $(\mu \mathrm{g} / \mathrm{ml})$ : & $2.43 E+00$ & $1.80 \mathrm{E}-02$ & \multirow{2}{*}{$\mathbf{v}$} \\
\hline & Weight of impurity in sample group $(\mu \mathrm{g})$ : & 96.96 & 0.68 & \\
\hline & Average concentration of impurity in ring blanks (ppmw): & 1.7266 & 0.0122 & 1.7387 \\
\hline
\end{tabular}

Weight of ash after nitric acid leaching was $0.0134 \mathrm{~g}$.

Comments

Data checked by FCM against the official results of analyses for RMAL3864 on 8/22/2011.

Fudvick C. Monteroney
$9-1-2011$

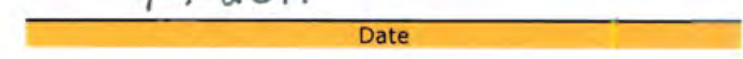


Data Report Form DRF-44: Measurement of Impurities in Matrix Ring Blanks by the Burn-Leach Method

\begin{tabular}{|c|c|}
\hline Procedure: & AGR-CHAR-DAM-44 Rev. 0 \\
\hline Operator: & Fred Montgomery \\
\hline Ring blank lot ID: & RDKRS-Machined \\
\hline Ring blank lot description: & Rings machined from blanks made using matrix blend with Hexion Durite $\mathrm{SC}-1008$ resin \\
\hline Ring ID numbers: & $2022, Z 023$ \\
\hline DRF filename: & ach \RDKRS-Machined_DRF44R0.xls \\
\hline
\end{tabular}

\begin{tabular}{|c|c|c|c|c|}
\hline & & First Leach & Second Leach & Total \\
\hline & Leach solution ID: & BL11071502 & BL11071802 & \\
\hline & Number of ring blanks in sample group: & & \\
\hline & Total weight of ring blanks in sample group & \multicolumn{2}{|c|}{57.0} & \\
\hline & Total volume of leach solution $(\mathrm{ml})$ : & 28.8 & 24.5 & \\
\hline & Radiochemical laboratory analysis number: & $3864-002$ & $3864-006$ & \\
\hline \multirow{3}{*}{$\mathbf{u}$} & Measured concentration of impurity in solution $(\mu \mathrm{g} / \mathrm{ml})$ : & $1.79 \mathrm{E}-02$ & $2.80 \mathrm{E}-04$ & \multirow{2}{*}{ u } \\
\hline & $\begin{array}{l}\text { Weight of impurity in sample group }(\mu \mathrm{g}) \text { : } \\
\text {. }\end{array}$ & 0.52 & 0.01 & \\
\hline & Average concentration of impurity in ring blanks ( $\mathrm{ppmw}$ ): & 0.0090 & 0.0001 & 0.0092 \\
\hline \multirow{3}{*}{$\mathbf{F e}$} & Measured concentration of impurity in solution $(\mu \mathrm{g} / \mathrm{ml})$ : & $1.15 E+00$ & $4.76 \mathrm{E}-02$ & \multirow{2}{*}{$\mathrm{Fe}$} \\
\hline & Weight of impurity in sample group $(\mu \mathrm{g})$ : & 33.12 & 1.17 & \\
\hline & Average concentration of impurity in ring blanks (ppmw): & 0.5814 & 0.0205 & 0.6019 \\
\hline \multirow{3}{*}{$\mathrm{Cr}$} & Measured concentration of impurity in solution $(\mu \mathrm{g} / \mathrm{ml})$ : & $7.96 \mathrm{E}-02$ & $1.03 E-02$ & \multirow{2}{*}{$\mathrm{Cr}$} \\
\hline & Weight of impurity in sample group $(\mu g)$ : & 2.29 & 0.25 & \\
\hline & Average concentration of impurity in ring blanks (ppmw): & 0.0402 & 0.0044 & 0.0447 \\
\hline \multirow{3}{*}{ Mn } & Measured concentration of impurity in solution $(\mu \mathrm{g} / \mathrm{ml})$ : & $1.05 \mathrm{E}-02$ & $<1.91 \mathrm{E}-03$ & \multirow{2}{*}{ Mn } \\
\hline & Weight of impurity in sample group $(\mu g)$ : & 0.30 & $<0.05$ & \\
\hline & Average concentration of impurity in ring blanks (ppmw): & 0.0053 & $<0.0008$ & 0.0061 \\
\hline \multirow{3}{*}{ Co } & Measured concentration of impurity in solution $(\mu \mathrm{g} / \mathrm{ml})$ : & $1.21 \mathrm{E}-02$ & $<1.62 \mathrm{E}-03$ & \multirow{2}{*}{ Co } \\
\hline & Weight of impurity in sample group $(\mu \mathrm{g})$ : & 0.35 & $<0.04$ & \\
\hline & Average concentration of impurity in ring blanks (ppmw): & 0.0061 & $<0.0007$ & 0.0068 \\
\hline \multirow{3}{*}{$\mathbf{N i}$} & Measured concentration of impurity in solution $(\mu \mathrm{g} / \mathrm{ml})$ : & $4.05 E-02$ & $<8.00 \mathrm{E}-03$ & \multirow{2}{*}{$\mathrm{Ni}$} \\
\hline & Weight of impurity in sample group $(\mu \mathrm{g})$ : & 1.17 & $<0.20$ & \\
\hline & Average concentration of impurity in ring blanks (ppmw): & 0.0205 & $<0.0034$ & 0.0239 \\
\hline \multirow{3}{*}{ Ca } & Measured concentration of impurity in solution $(\mu \mathrm{g} / \mathrm{ml})$ : & $8.48 E+00$ & $5.60 \mathrm{E}-01$ & \multirow{2}{*}{$\mathrm{Ca}$} \\
\hline & Weight of impurity in sample group $(\mu \mathrm{g})$ : & 244.22 & 13.72 & \\
\hline & Average concentration of impurity in ring blanks (ppmw): & 4.2872 & 0.2408 & 4.5281 \\
\hline \multirow{3}{*}{ Al } & Measured concentration of impurity in solution $(\mu \mathrm{g} / \mathrm{ml})$ : & $4.77 E+00$ & $2.69 \mathrm{E}-01$ & \multirow{2}{*}{ Al } \\
\hline & Weight of impurity in sample group $(\mu \mathrm{g})$ : & 137.38 & 6.59 & \\
\hline & Average concentration of impurity in ring blanks (ppmw): & 2.4116 & 0.1157 & 2.5273 \\
\hline \multirow{3}{*}{$\mathbf{T i}$} & Measured concentration of impurity in solution $(\mu \mathrm{g} / \mathrm{ml})$ : & $1.35 E+00$ & $3.12 \mathrm{E}-01$ & \multirow[b]{2}{*}{$\mathrm{Ti}$} \\
\hline & Weight of impurity in sample group $(\mu \mathrm{g})$ : & 38.88 & 7.64 & \\
\hline & Average concentration of impurity in ring blanks (ppmw): & 0.6825 & 0.1342 & 0.8167 \\
\hline \multirow{3}{*}{$\mathbf{v}$} & Measured concentration of impurity in solution $(\mu \mathrm{g} / \mathrm{ml})$ : & $3.48 \mathrm{E}+00$ & $3.93 \mathrm{E}-01$ & \multirow{2}{*}{$\mathbf{v}$} \\
\hline & Weight of impurity in sample group $(\mu \mathrm{g})$ : & 100.22 & 9.63 & \\
\hline & Average concentration of impurity in ring blanks (ppmw): & 1.7594 & 0.1690 & $1 . \overline{9284}$ \\
\hline
\end{tabular}

Weight of ash after nitric acid leaching was $0.0131 \mathrm{~g}$.

Comments

Data checked by FCM against the official results of analyses for RMAL3864 on 8/22/2011.

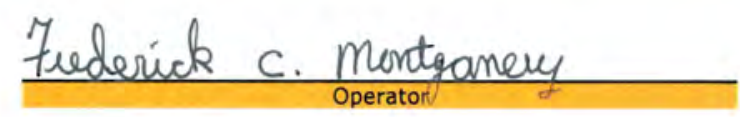

$9-1 \cdot 2011$ Operator

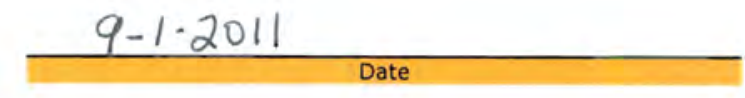


Data Report Form DRF-44: Measurement of Impurities in Matrix Ring Blanks by the Burn-Leach Method

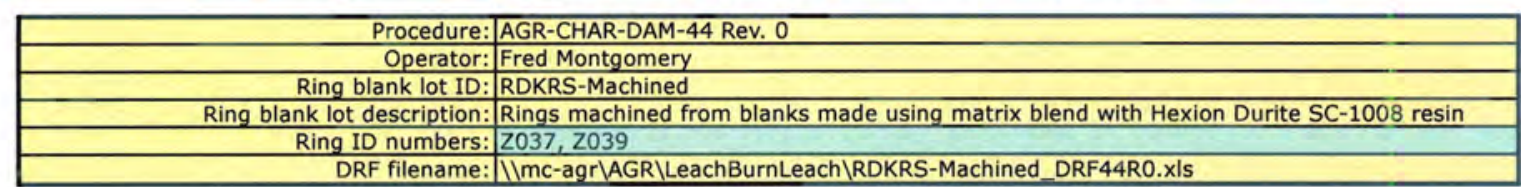

\begin{tabular}{|c|c|c|c|c|}
\hline & & First Leach & Second Leach & Total \\
\hline & Leach solution ID: & BL11071503 & BL11071803 & \\
\hline & Number of ring blanks in sample group: & & \\
\hline & Total weight of ring blanks in sample group & \multicolumn{2}{|c|}{57.0} & \\
\hline & Total volume of leach solution $(\mathrm{ml})$ : & 30.0 & 25.0 & \\
\hline & Radiochemical laboratory analysis number: & $3864-003$ & 3864-007 & \\
\hline \multirow{3}{*}{ u } & Measured concentration of impurity in solution $(\mu \mathrm{g} / \mathrm{ml})$ : & $2.53 \mathrm{E}-02$ & $9.72 \mathrm{E}-03$ & \multirow{2}{*}{$\mathbf{u}$} \\
\hline & Weight of impurity in sample group $(\mu \mathrm{g})$ : & 0.76 & 0.24 & \\
\hline & Average concentration of impurity in ring blanks (ppmw): & 0.0133 & 0.0043 & 0.0176 \\
\hline \multirow{3}{*}{$\mathbf{F e}$} & Measured concentration of impurity in solution $(\mu \mathrm{g} / \mathrm{ml})$ : & $6.40 \mathrm{E}-01$ & $<4.12 \mathrm{E}-02$ & \multirow{2}{*}{$\mathrm{Fe}$} \\
\hline & Weight of impurity in sample group $(\mu \mathrm{g})$ : & 19.20 & $<1.03$ & \\
\hline & Average concentration of impurity in ring blanks (ppmw): & 0.3369 & $<0.0181$ & 0.3550 \\
\hline \multirow{3}{*}{$\mathbf{C r}$} & Measured concentration of impurity in solution $(\mu \mathrm{g} / \mathrm{ml})$ : & $5.33 \mathrm{E}-02$ & $5.01 \mathrm{E}-03$ & \multirow{2}{*}{$\mathrm{Cr}$} \\
\hline & Weight of impurity in sample group $(\mu \mathrm{g})$ : & 1.60 & 0.13 & \\
\hline & Average concentration of impurity in ring blanks (ppmw): & 0.0281 & 0.0022 & 0.0303 \\
\hline \multirow{3}{*}{ Mn } & Measured concentration of impurity in solution $(\mu \mathrm{g} / \mathrm{ml})$ : & $7.51 \mathrm{E}-03$ & $<1.91 \mathrm{E}-03$ & \multirow{2}{*}{ Mn } \\
\hline & Weight of impurity in sample group $(\mu \mathrm{g})$ : & 0.23 & $<0.05$ & \\
\hline & Average concentration of impurity in ring blanks (ppmw): & 0.0040 & $<0.0008$ & 0.0048 \\
\hline \multirow{3}{*}{ Co } & Measured concentration of impurity in solution $(\mu \mathrm{g} / \mathrm{ml})$ : & $6.55 \mathrm{E}-03$ & $<1.62 \mathrm{E}-03$ & \multirow{2}{*}{ Co } \\
\hline & Weight of impurity in sample group $(\mu \mathrm{g})$ : & 0.20 & $<0.04$ & \\
\hline & Average concentration of impurity in ring blanks (ppmw): & 0.0034 & $<0.0007$ & 0.0042 \\
\hline \multirow{3}{*}{$\mathbf{N i}$} & Measured concentration of impurity in solution $(\mu \mathrm{g} / \mathrm{ml})$ : & $3.03 E-02$ & $<8.00 \mathrm{E}-03$ & \multirow{2}{*}{$\mathrm{Ni}$} \\
\hline & Weight of impurity in sample group $(\mu \mathrm{g})$ : & 0.91 & $<0.20$ & \\
\hline & Average concentration of impurity in ring blanks (ppmw): & 0.0160 & $<0.0035$ & 0.0195 \\
\hline \multirow{3}{*}{$\mathbf{C a}$} & Measured concentration of impurity in solution $(\mu \mathrm{g} / \mathrm{ml})$ : & $1.12 \mathrm{E}+01$ & $8.69 \mathrm{E}-01$ & \multirow{2}{*}{$\mathrm{Ca}$} \\
\hline & Weight of impurity in sample group $(\mu \mathrm{g})$ : & 336.00 & 21.73 & \\
\hline & Average concentration of impurity in ring blanks (ppmw): & 5.8960 & 0.3812 & 6.2772 \\
\hline \multirow{3}{*}{ AI } & Measured concentration of impurity in solution $(\mu \mathrm{g} / \mathrm{ml})$ : & $3.47 E+00$ & $2.28 \mathrm{E}-01$ & \multirow[b]{2}{*}{ AI } \\
\hline & Weight of impurity in sample group $(\mu g)$ : & 104.10 & 5.70 & \\
\hline & Average concentration of impurity in ring blanks (ppmw): & 1.8267 & 0.1000 & 1.9267 \\
\hline \multirow{3}{*}{$\mathbf{T i}$} & Measured concentration of impurity in solution $(\mu \mathrm{g} / \mathrm{ml})$ : & $1.39 \mathrm{E}+00$ & $1.93 \mathrm{E}-02$ & \multirow{2}{*}{ Ti } \\
\hline & Weight of impurity in sample group $(\mu \mathrm{g})$ : & 41.70 & 0.48 & \\
\hline & Average concentration of impurity in ring blanks (ppmw): & 0.7317 & 0.0085 & $0 . \overline{7402}$ \\
\hline \multirow{3}{*}{$\mathbf{v}$} & Measured concentration of impurity in solution $(\mu \mathrm{g} / \mathrm{ml})$ : & $3.27 E+00$ & 7.87E-03 & \multirow[b]{2}{*}{$\mathbf{v}$} \\
\hline & Weight of impurity in sample group $(\mu g)$ : & 98.10 & 0.20 & \\
\hline & Average concentration of impurity in ring blanks (ppmw): & 1.7214 & 0.0035 & 1.7249 \\
\hline \multicolumn{5}{|c|}{ Comments } \\
\hline \multicolumn{5}{|c|}{$\begin{array}{l}\text { Weight of ash after nitric acid leaching was } 0.0161 \mathrm{~g} \text {. } \\
\text { Data checked by FCM against the official results of analyses for RMAL3864 on 8/22/2011. }\end{array}$} \\
\hline
\end{tabular}

Zuelaick c. mintomuy
$9-|-20| \mid$

Date 
Data Report Form DRF-44 Supplemental: Measurement of Impurities in Matrix Ring Blank Ash

\begin{tabular}{|c|c|}
\hline Procedure: & AGR-CHAR-DAM-44 Rev. 0 \\
\hline Operator: & Fred Montgomery \\
\hline Ring blank lot ID: & RDKRS-Machined \\
\hline Ring blank lot description: & Rings machined from blanks made using matrix blend with Hexion Durite SC- 1008 resin \\
\hline Ring blank ID numbers: & 2005,2010 \\
\hline DRF filename: & chlRDKRS-Machined_DRF44R0.xis \\
\hline
\end{tabular}

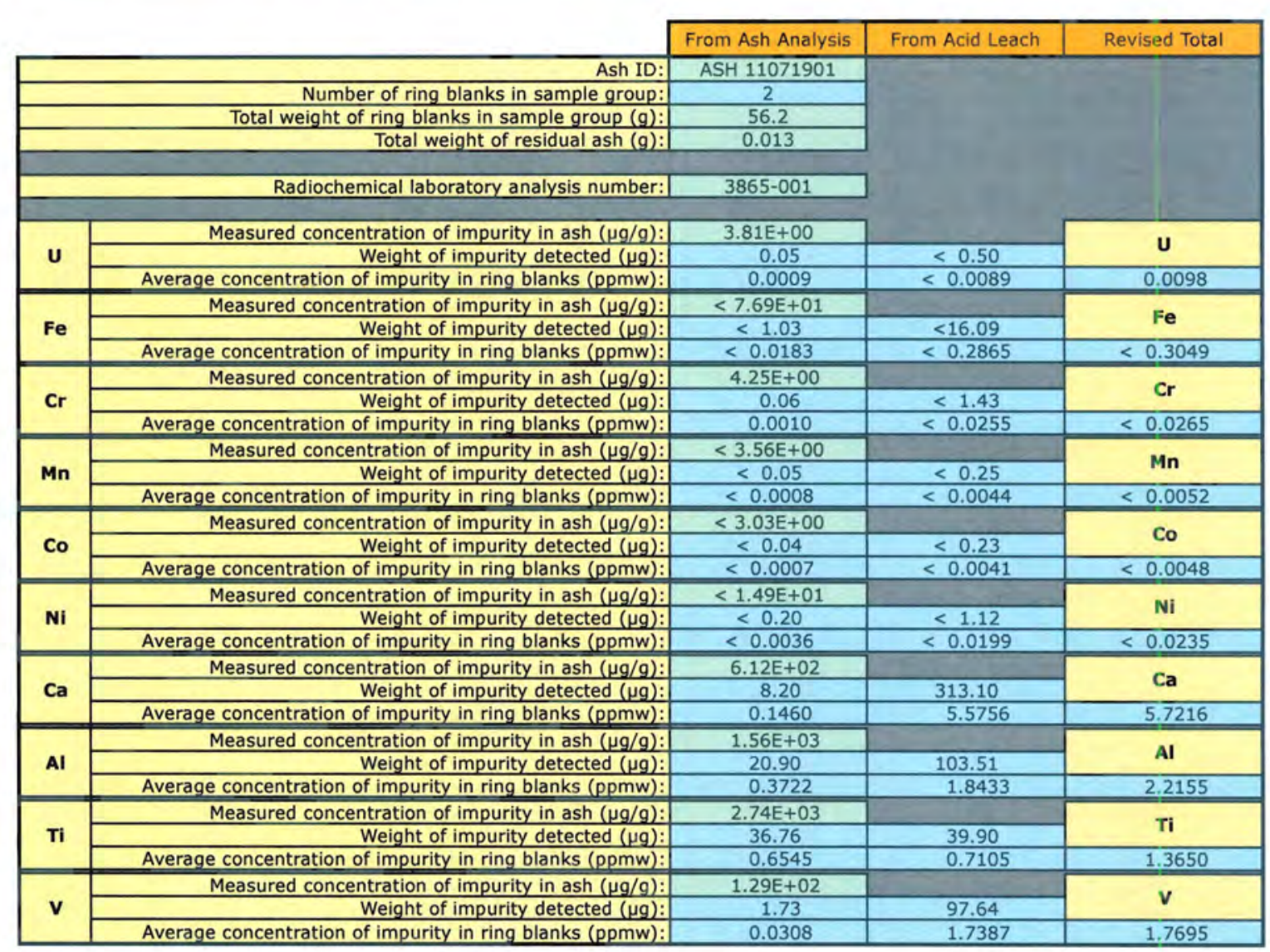

Additional ring blank impurities greater than about 1 ppmw detected in ash: 78 ppmw Si, 4.5 ppmw Ba. Data checked by FCM against the official results of analyses for RMAL3865 on 8/22/2011.

Fied c. montromery
$9-(-201)$ 
Data Report Form DRF-44 Supplemental: Measurement of Impurities in Matrix Ring Blank Ash

\begin{tabular}{|r|l|}
\hline Procedure: & AGR-CHAR-DAM-44 Rev. 0 \\
\hline Operator: & Fred Montgomery \\
\hline Ring blank lot ID: & RDKRS-Machined \\
\hline Ring blank lot description: & Rings machined from blanks made using matrix blend with Hexion Durite SC-1008 resin \\
\hline Ring blank ID numbers: & Z022, Z023 \\
\hline DRF filename: & \Imc-agr|AGRILeachBurnLeach \RDKRS-Machined_DRF44R0.xls \\
\hline
\end{tabular}

\begin{tabular}{|c|c|c|c|c|}
\hline & From Ash Analysis & From Acid Leach & Revised Total \\
\hline \multicolumn{2}{|r|}{ Ash ID: } & ASH 11071902 & & \\
\hline \multicolumn{2}{|r|}{ Number of ring blanks in sample group: } & 2 & & \\
\hline \multirow{2}{*}{\multicolumn{2}{|c|}{$\begin{array}{r}\text { Total weight of ring blanks in sample group }(\mathrm{g}) \text { : } \\
\text { Total weight of residual ash }(\mathrm{g}):\end{array}$}} & 57.0 & & \\
\hline & & 0.013 & & \\
\hline \multicolumn{2}{|r|}{ Radiochemical laboratory analysis number: } & $3865-002$ & & \\
\hline \multirow{3}{*}{$\mathbf{u}$} & & & & \multirow{2}{*}{ u } \\
\hline & $\frac{\text { Measured concentration of impurity in ash }(\mu \mathrm{g} / \mathrm{g}) \text { : }}{\text { Weiaht of impurity detected }(\mu \mathrm{q}):}$ & $\frac{3.28 \mathrm{E}+00}{0.04}$ & & \\
\hline & Average concentration of impurity in ring blanks (ppmw): & 0.0008 & 0.0092 & 0.0099 \\
\hline \multirow{3}{*}{$\mathbf{F e}$} & Measured concentration of impurity in ash $(\mu \mathrm{g} / \mathrm{g})$ : & $1.77 E+02$ & & \multirow{2}{*}{$\mathrm{Fe}$} \\
\hline & Weight of impurity detected $(\mu g)$ : & 2.32 & 34.29 & \\
\hline & Average concentration of impurity in ring blanks (ppmw): & 0.0407 & 0.6019 & 0.6426 \\
\hline \multirow{3}{*}{$\mathrm{Cr}$} & Measured concentration of impurity in ash $(\mu \mathrm{g} / \mathrm{g})$ : & $1.26 \mathrm{E}+01$ & & \multirow{2}{*}{$\mathrm{Cr}$} \\
\hline & Weight of impurity detected $(\mu \mathrm{g})$ : & 0.17 & 2.54 & \\
\hline & Average concentration of impurity in ring blanks (ppmw): & 0.0029 & 0.0447 & 0.0476 \\
\hline \multirow{3}{*}{ Mn } & Measured concentration of impurity in ash $(\mu \mathrm{g} / \mathrm{g})$ : & $<3.64 E+00$ & & \multirow[b]{2}{*}{ Mn } \\
\hline & Weight of impurity detected $(\mu \mathrm{g})$ : & $<0.05$ & $<0.35$ & \\
\hline & Average concentration of impurity in ring blanks (ppmw): & $<0.0008$ & $<0.0061$ & $<0.0070$ \\
\hline \multirow{3}{*}{ Co } & Measured concentration of impurity in ash $(\mu g / g)$ : & $<3.10 E+00$ & & \multirow[b]{2}{*}{ Co } \\
\hline & Weight of impurity detected $(\mu \mathrm{g})$ : & $<0.04$ & $<0.39$ & \\
\hline & Average concentration of impurity in ring blanks (ppmw): & $<0.0007$ & $<0.0068$ & $<0.0075$ \\
\hline \multirow{3}{*}{$\mathbf{N i}$} & Measured concentration of impurity in ash $(\mu \mathrm{g} / \mathrm{g})$ : & $<1.53 E+01$ & & \multirow{2}{*}{ Ni } \\
\hline & Weight of impurity detected $(\mu \mathrm{g})$ : & $<0.20$ & $<1.36$ & \\
\hline & Average concentration of impurity in ring blanks (ppmw): & $<0.0035$ & $<0.0239$ & $<0.0274$ \\
\hline \multirow{3}{*}{$\mathbf{C a}$} & Measured concentration of impurity in ash $(\mu \mathrm{g} / \mathrm{g})$ : & $4.85 E+02$ & & \multirow[b]{2}{*}{ Ca } \\
\hline & Weight of impurity detected $(\mu \mathrm{g})$ : & 6.35 & 257.94 & \\
\hline & Average concentration of impurity in ring blanks (ppmw): & 0.1115 & 4.5281 & 4.6396 \\
\hline \multirow{3}{*}{ Al } & Measured concentration of impurity in ash $(\mu \mathrm{g} / \mathrm{g})$ : & $1.31 \mathrm{E}+03$ & & \multirow[b]{2}{*}{ A! } \\
\hline & Weight of impurity detected $(\mu \mathrm{g})$ : & 17.16 & 143.97 & \\
\hline & Average concentration of impurity in ring blanks (ppmw): & 0.3013 & 2.5273 & 2.8285 \\
\hline \multirow{3}{*}{$\mathbf{T i}$} & Measured concentration of impurity in ash $(\mu \mathrm{g} / \mathrm{g})$ : & $2.14 E+03$ & & \multirow{2}{*}{$\mathrm{Ti}$} \\
\hline & Weight of impurity detected $(\mu \mathrm{g})$ : & 28.03 & 46.52 & \\
\hline & Average concentration of impurity in ring blanks (ppmw): & 0.4921 & 0.8167 & 1.3088 \\
\hline \multirow{3}{*}{$\mathbf{v}$} & Measured concentration of impurity in ash $(\mu \mathrm{g} / \mathrm{g})$ : & $1.13 E+02$ & & \multirow{2}{*}{ v } \\
\hline & Weight of impurity detected $(\mu \mathrm{g})$ : & 1.48 & 109.85 & \\
\hline & Average concentration of impurity in ring blanks (ppmw): & 0.0260 & 1.9284 & 1.9544 \\
\hline
\end{tabular}

\section{Comments}

Additional ring blank impurities greater than about 1 ppmw detected in ash: $80 \mathrm{ppmw} \mathrm{Si,} 1.8 \mathrm{ppmw} \mathrm{Ba}$. Data checked by FCM against the official results of analyses for RMAL3865 on 8/22/2011.

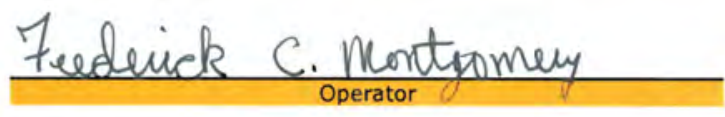


Data Report Form DRF-44 Supplemental: Measurement of Impurities in Matrix Ring Blank Ash

\begin{tabular}{|c|c|}
\hline Procedure: & AGR-CHAR-DAM-44 Rev. 0 \\
\hline Operator: & Fred Montgomery \\
\hline Ring blank lot ID: & RDKRS-Machined \\
\hline Ring blank lot description: & Rings machined from blanks made using matrix blend with Hexion Durite SC- 1008 resin \\
\hline Ring blank ID numbers: & 2037,2039 \\
\hline DRF filename: & IImc-agr\AGR\LeachBurnLeach\RDKRS-Machined_DRF44R0.xls \\
\hline
\end{tabular}

\begin{tabular}{|c|c|c|c|c|}
\hline & \multirow[b]{2}{*}{ Ash ID } & \multirow{2}{*}{$\frac{\text { From Ash Analysis }}{\text { ASH } 11071903}$} & \multirow[t]{2}{*}{ From Acid Leach } & Revised Total \\
\hline & & & & \\
\hline & Number of ring blanks in sample group: & 2 & & \\
\hline & Total weight of ring blanks in sample group (g): & 57.0 & & \\
\hline & Total weight of residual ash $(\mathrm{g})$ : & 0.016 & & \\
\hline \multicolumn{2}{|r|}{ Radiochemical laboratory analysis number: } & $3865-003$ & & \\
\hline \multirow{3}{*}{$\mathbf{u}$} & Measured concentration of impurity in ash $(\mu \mathrm{g} / \mathrm{g})$ : & $4.97 E+00$ & & \multirow[b]{2}{*}{$\mathbf{u}$} \\
\hline & Weight of impurity detected $(\mu \mathrm{g})$ : & 0.08 & 1.00 & \\
\hline & Average concentration of impurity in ring blanks (ppmw): & 0.0014 & 0.0176 & 0.0190 \\
\hline \multirow{3}{*}{$\mathbf{F e}$} & Measured concentration of impurity in ash $(\mu \mathrm{g} / \mathrm{g})$ : & $<6.40 \mathrm{E}+01$ & & \multirow[b]{2}{*}{$\mathrm{Fe}$} \\
\hline & Weight of impurity detected $(\mu \mathrm{g})$ : & $<1.03$ & $<20.23$ & \\
\hline & Average concentration of impurity in ring blanks (ppmw): & $<0.0181$ & $<0.3550$ & $<0.3731$ \\
\hline \multirow{3}{*}{ Cr } & Measured concentration of impurity in ash $(\mu \mathrm{g} / \mathrm{g})$ : & $6.08 \mathrm{E}+00$ & & \multirow{2}{*}{$\mathrm{Cr}$} \\
\hline & Weight of impurity detected $(\mu \mathrm{g})$ : & 0.10 & 1.72 & \\
\hline & Average concentration of impurity in ring blanks (ppmw): & 0.0017 & 0.0303 & 0.0320 \\
\hline \multirow{3}{*}{ Mn } & Measured concentration of impurity in ash $(\mu \mathrm{g} / \mathrm{g})$ : & $<2.96 \mathrm{E}+00$ & & \multirow{2}{*}{ Mn } \\
\hline & Weight of impurity detected $(\mu \mathrm{g})$ : & $<0.05$ & $<0.27$ & \\
\hline & Average concentration of impurity in ring blanks (ppmw): & $<0.0008$ & $<0.0048$ & $<0.0056$ \\
\hline \multirow{3}{*}{ Co } & Measured concentration of impurity in ash $(\mu \mathrm{g} / \mathrm{g})$ : & $<2.52 \mathrm{E}+00$ & & \multirow{2}{*}{ Co } \\
\hline & Weight of impurity detected $(\mu \mathrm{g})$ : & $<0.04$ & $<0.24$ & \\
\hline & Average concentration of impurity in ring blanks (ppmw): & $<0.0007$ & $<0.0042$ & $<0.0049$ \\
\hline \multirow{3}{*}{$\mathbf{N i}$} & Measured concentration of impurity in ash $(\mu \mathrm{g} / \mathrm{g})$ : & $<1.24 \mathrm{E}+01$ & & \multirow{2}{*}{ Ni } \\
\hline & Weight of impurity detected $(\mu \mathrm{g})$ : & $<0.20$ & $<1.11$ & \\
\hline & Average concentration of impurity in ring blanks (ppmw): & $<0.0035$ & $<0.0195$ & $<0.0230$ \\
\hline \multirow{3}{*}{ Ca } & Measured concentration of impurity in ash $(\mu \mathrm{g} / \mathrm{g})$ : & $3.42 \mathrm{E}+02$ & & \multirow{2}{*}{$\mathrm{Ca}$} \\
\hline & Weight of impurity detected $(\mu \mathrm{g})$ : & 5.51 & 357.73 & \\
\hline & Average concentration of impurity in ring blanks (ppmw): & 0.0966 & 6.2772 & 6.3738 \\
\hline \multirow{3}{*}{ Al } & Measured concentration of impurity in ash $(\mu \mathrm{g} / \mathrm{g})$ : & $1.16 \mathrm{E}+03$ & & \multirow[b]{2}{*}{ Al } \\
\hline & Weight of impurity detected $(\mu \mathrm{g})$ : & 18.68 & 109.80 & \\
\hline & Average concentration of impurity in ring blanks (ppmw): & 0.3277 & 1.9267 & 2.2544 \\
\hline \multirow{3}{*}{$\mathbf{T i}$} & Measured concentration of impurity in ash $(\mu \mathrm{g} / \mathrm{g})$ : & $7.42 \mathrm{E}+02$ & & \multirow{2}{*}{$\pi$} \\
\hline & Weight of impurity detected $(\mu \mathrm{g})$ : & 11.95 & 42.18 & \\
\hline & Average concentration of impurity in ring blanks (ppmw): & 0.2096 & 0.7402 & 0.9498 \\
\hline \multirow{3}{*}{$\mathbf{v}$} & Measured concentration of impurity in ash $(\mu \mathrm{g} / \mathrm{g})$ : & $7.19 E+01$ & & \multirow{2}{*}{ v } \\
\hline & Weight of impurity detected $(\mu \mathrm{g})$ : & 1.16 & 98.30 & \\
\hline & Average concentration of impurity in ring blanks (ppmw): & 0.0203 & 1.7249 & 1.7452 \\
\hline
\end{tabular}

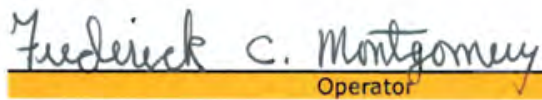




\section{Appendix A. Revision Log}

Table 7. Changes made in Revision 1

\begin{tabular}{|c|l|}
\hline Pages & \multicolumn{1}{c|}{ Description of change } \\
\hline 4 & Updated Table of Contents. \\
\hline 15 & Modified last paragraph to reflect completion of ash residue impurity analysis. \\
\hline $33-38$ & Added Section 6 to report ash residue impurity analysis results. \\
\hline $39-47$ & Added Section 7 to report machined matrix ring impurity analysis results. \\
\hline 48 & Added Appendix A to track changes. \\
\hline & \\
\hline & \\
\hline
\end{tabular}

